\title{
Geminal Diol Formation from the Interaction of a Ketone with Water in the Gas Phase: Structure and Reactivity of Cyclooctanone- $\left(\mathrm{H}_{2} \mathrm{O}\right)_{1,2}$ Clusters
}

\author{
Ecaterina Burevschi, Isabel Peña†, M. Eugenia Sanz* \\ Department of Chemistry, King's College London \\ London SE1 1DB, United Kingdom
}

[†] Present Address: Departamento de Química Física y Química Inorgánica, Universidad de Valladolid, E-47011 Valladolid, Spain.

Corresponding Author

*E-mail:maria.sanz@kcl.ac.uk 


\section{Table of Contents}

Methods

Theoretical methods

Experimental methods

\section{Spectral Assignment}

Table S1. Theoretical spectroscopic parameters and relative energies for the isomers of cyclooctanone- $\mathrm{H}_{2} \mathrm{O}$ within $900 \mathrm{~cm}^{-1}$.

Table S2. Theoretical spectroscopic parameters and relative energies for the isomers of cyclooctanone- $\left(\mathrm{H}_{2} \mathrm{O}\right)_{2}$ within $800 \mathrm{~cm}^{-1}$.

Table S3. Experimental spectroscopic parameters for all singly substituted ${ }^{13} \mathrm{C}$ and ${ }^{18} \mathrm{O}$ isotopic species of the observed cyclooctanone- $\left(\mathrm{H}_{2} \mathrm{O}\right)_{1,2}$ complexes.

Table S4-S7. Substitution coordinates of the heavy atoms of the $\mathbf{1} \mathbf{w}-\mathbf{I}, \mathbf{1} \mathbf{w}-\mathbf{I I}, \mathbf{2} \mathbf{w}-\mathbf{I}$ and $\mathbf{2} \mathbf{w}-\mathbf{I V}$ complexes of cyclooctanone- $\mathrm{H}_{2} \mathrm{O}$ in $\AA$, and comparison with the MP2/6-311++G(d,p) values of possible matches (see text for full explanation).

Table S8. B3LYP-D3BJ and MP2 spectroscopic parameters and relative energies for 1,1-cycloctanediol within $1000 \mathrm{~cm}^{-1}$.

Table S9. Experimental and theoretical bond lengths (in $\AA$ ) and angles (in degrees) of cyclooctanone- $\mathrm{H}_{2} \mathrm{O}$ and comparison with the experimental structural parameters of bare cyclooctanone.

Table S10. Experimental and theoretical bond lengths (in $\AA$ ) and angles (in degrees) of cyclooctanone- $\left(\mathrm{H}_{2} \mathrm{O}\right)_{2}$ and comparison with the experimental structural parameters of bare cyclooctanone.

Table S11. Binding energy decomposition analysis in $\mathrm{kJ} \mathrm{mol}^{-1}$ for the complexes of cyclooctanone- $\mathrm{H}_{2} \mathrm{O}$ on their MP2 and B3LYP-D3BJ geometries, using SAPT(0)/jun-cc-pDVZ calculations within Psi4. The overall binding energies are in agreement with those obtained from B3LYP-D3BJ and MP2 calculations.

Table S12. B3LYP-D3BJ and MP2 spectroscopic parameters and relative energies for the 1,1-cycloctanediol$\mathrm{H}_{2} \mathrm{O}$ complex within $1000 \mathrm{~cm}^{-1}$.

Table S13. MP2/6-311++G(d,p) values for the equilibrium, zero-point energies, and Gibbs energies (346 K, 1 atm) for cyclooctanone $\mathrm{BC} 1$, its observed complexes with water and the corresponding gem-diols and transition states.

Figure S1. Top view of some cyclooctanone- $\mathrm{H}_{2} \mathrm{O}$ structures predicted differently from B3LYP-D3BJ and MP2 calculations.

Figure S2. Overlays of the MP2/6-311++G(d,p) structures of the observed isomers of cyclooctanone- $\left(\mathrm{H}_{2} \mathrm{O}\right)_{1,2}$ with the $r_{s}$ positions of the atoms of water from experimental observations (blue spheres).

Figure S3. Plots of the reduced density gradient (RDG) versus $\operatorname{sign}(\lambda 2) \rho$ for the observed complexes of cyclooctanone- $\mathrm{H}_{2} \mathrm{O}$.

\section{Frequency tables}

Table S14-S59. Measured frequencies and residuals (in $\mathrm{MHz}$ ) for the rotational transitions of the observed complexes of cyclooctanone- $\left(\mathrm{H}_{2} \mathrm{O}\right)_{1,2}$ and the ${ }^{13} \mathrm{C}$ and ${ }^{18} \mathrm{O}$ isotopologues. 


\section{Methods}

\section{Theoretical}

The complexes of cyclooctanone with one and two water molecules were predicted using the CREST software, ${ }^{1}$ with conformers $\mathbf{B C 1}, \mathbf{B C 2}$, TBC1 and $\mathbf{C R}$ of cyclooctanone ${ }^{2}$ as initial structures for the configurational search. The resulting isomers were optimised at B3LYP-D3BJ and MP2 levels of theory with the $6-311++G(d, p)$ basis set. All isomers were confirmed to be local minima by performing harmonic vibrational calculations, and their zero-point relative energies were obtained. Dissociation energies using the counterpoise method, ${ }^{3}$ including fragment relaxation terms, ${ }^{4}$ were determined (Tables S1, S2). Energy decomposition analysis with symmetry-adapted perturbation theory (SAPT) for the complexes with one water molecule was also performed (Table S11).

Both B3LYP-D3BJ and MP2 methods agree in their predicted global minima and the general energy ordering, although there are some discrepancies for higher-energy complexes. For some isomers there are inconsistencies in the predicted rotational constants because the optimised structures are slightly different for each method (see Fig. S1). For example, the predicted MP2 configuration for isomer $\mathbf{1} \mathbf{w}-\mathbf{I}$ has the water molecule shifted closer to the centre of the cyclooctanone ring in comparison with B3LYP-D3BJ predictions. A similar behaviour is observed for $\mathbf{1} \mathbf{w}-\mathbf{I V}$ and $\mathbf{1} \mathbf{w}-\mathbf{V}$. The differences between the rotational constantans and the dipole moments predicted by the two methods are particularly large for $\mathbf{1 w}$-IV.

\section{Experimental}

The spectrum of cyclooctanone with water was collected using our chirped-pulse Fourier transform microwave spectrometer at King's College London, which operates in the 2-8 GHz frequency range. ${ }^{5,6}$ Cyclooctanone $(98 \%)$ is a crystalline solid at room temperature, with a melting point of $313 \mathrm{~K}$. In order to increase its concentration in the gas phase, the sample was placed in a bespoke heated nozzle and heated to $346 \mathrm{~K}$, which was found to be the optimal temperature after several tests. Water was added by placing it in a receptacle outside the vacuum chamber and passing neon over it at stagnation pressures of 5 bar. The mixture of cyclooctanone and water was conducted to the vacuum chamber, where it forms a supersonic jet after going through a $1 \mathrm{~mm}$ diameter nozzle. The molecular pulse duration in the experimental setup was $1000 \mu \mathrm{s}$. The species in the supersonic jet were polarised with 4 chirped microwave pulses of $4 \mu$ s length varying linearly in frequency from 2 to $8 \mathrm{GHz}$. After each excitation pulse, molecular relaxation signals (free inductions decays, FIDs) were collected for $20 \mu$ s by our digital oscilloscope. The time domain spectrum was converted to the frequency domain using a fast Fourier-transform algorithm with a Kaiser-Bessel window. 1.4 million FIDs were coherently added to produce our final spectrum. The spectrum of cyclooctanone with ${ }^{18} \mathrm{O}$ isotopically enriched water $\left(1: 1\right.$ mixture of $\mathrm{H}_{2}{ }^{16} \mathrm{O}$ and $\left.\mathrm{H}_{2}{ }^{18} \mathrm{O}\right)$ was recorded to determine the experimental positions of the oxygen atoms in the complexes. 


\section{Spectral Assignment}

$1 w-I$

The experimental rotational constants and observed relative intensities of $a$ - and $c$-type transitions of this species could match those predicted for $\mathbf{1} \mathbf{w}-\mathbf{I}$ and $\mathbf{1} \mathbf{w}-\mathbf{V}$. However, the much higher relative energy predicted for $\mathbf{1} \mathbf{w}-\mathbf{V}$ points to $\mathbf{1} \mathbf{w}-\mathbf{I}$ (the theoretical global minimum) as the complex in our supersonic jet. Definite confirmation was obtained from the observation of all the ${ }^{13} \mathrm{C}$ isotopologues in their natural abundance, and determination of the substitution coordinates using Kraitchman's equations ${ }^{7,8}$ (Table S4).

$1 w-I I$

The experimental rotational constants of the second observed isomer of $\mathrm{CYO}-\mathrm{H}_{2} \mathrm{O}$ match the theoretical rotational constants of $\mathbf{1} \mathbf{w}$-II. The observed intensity of the $a-, b$ - and $c$-type transitions is also consistent with the predicted dipole moment components for $\mathbf{1 w}$-II. The assignment was confirmed by the observation of all ${ }^{13} \mathrm{C}$ and ${ }^{18} \mathrm{O}$ isotopologues at the predicted frequency shifts and the agreement of the experimental $r_{s}$ coordinates of the heavy atoms with the theoretical ones (Table S5).

$2 w-1$

The experimental rotational constants of this species could be matched with the theoretical rotational constants for complexes $\mathbf{2} \mathbf{w}-\mathbf{I}$ and $\mathbf{2 w}-\mathbf{I I}$, where the arrangement of the water molecules is very similar. The only difference between the two predicted complexes is the orientation of the hydrogen atoms in the water molecules which are not part of the hydrogen bonding. This causes a slight energy difference and a larger difference in the $\mu_{c}$ dipole moment component, which helped identify the observed species. The assigned isomer showed mainly $a$-type spectrum with a strong intensity and $c$-type lines with approximately seven times lower intensity compared to the $a$-type lines, which is in better agreement with the predicted $\mu_{a}$ and $\mu_{c}$ dipole moments of the $\mathbf{2} \mathbf{w}-\mathbf{I}$ complex. $\mathbf{2 w}$-II is expected to show $a$ - and $c$-type spectra with similar intensities according to the MP2 predicted dipole moments, or $a$-type spectrum twice as intense compared to the $c$-type spectrum at B3LYP-D3BJ level. It is not possible to distinguish between $\mathbf{2} \mathbf{w}-\mathbf{I}$ and $\mathbf{2} \mathbf{w}-\mathrm{II}$ using the heavy atom coordinates (Table S6) as they only differ in the position of the hydrogen atoms of the water molecules.

Other complexes with similar predicted rotational constants are $\mathbf{2} \mathbf{w}-\mathbf{I X}$ and $\mathbf{2} \mathbf{w}-\mathbf{X}$. These are much higher in energy and correspond to configurations where the water molecules bind to the other lone pair of the carbonyl oxygen. From the values of the heavy atoms $r_{\mathrm{s}}$ coordinates (Table S6), determined from the observed monosubstituted ${ }^{13} \mathrm{C}$ and ${ }^{18} \mathrm{O}$ isotopologues, we can rule out $\mathbf{2} \mathbf{w}-\mathbf{I X}$ and $\mathbf{2} \mathbf{w}-\mathbf{X}$ as the species contributing to the spectrum.

\section{2w-IV}

The experimental rotational constants of the second isomer of $\mathrm{CYO}-\left(\mathrm{H}_{2} \mathrm{O}\right)_{2}$ (see Table 2) are similar to those predicted for complexes $\mathbf{2} \mathbf{w}-\mathbf{V}, \mathbf{2} \mathbf{w}-\mathbf{I I I}$ and $\mathbf{2} \mathbf{w}-\mathbf{I V}$. The differences between the three complexes are the orientation of the non-bonding hydrogens in the water molecules. The fact that we could not observed $b$-type transitions allows us to discard $\mathbf{2 w - I I I , ~ w i t h ~ a ~ s i z a b l e ~ t h e o r e t i c a l ~} \mu_{b}$ dipole moment of 0.9 D (Table S2). 
The data from the isotopic species, in particular the coordinates of the ${ }^{18} \mathrm{O}$, allowed us to discriminate between $\mathbf{2} \mathbf{w}-\mathbf{I V}$ and $\mathbf{2} \mathbf{w}-\mathbf{V}$, confirming the observed species to be $\mathbf{2} \mathbf{w}-\mathbf{I V}$ (Table S7).

\section{References}

(1) Pracht, P.; Bohle, F.; Grimme, S. Automated Exploration of the Low-Energy Chemical Space with Fast Quantum Chemical Methods. Phys. Chem. Chem. Phys. 2020, 22, 7169-7192. https://doi.org/10.1039/c9cp06869d.

(2) Burevschi, E.; Peña, I.; Sanz, M. E. Medium-Sized Rings: Conformational Preferences in Cyclooctanone Driven by Transannular Repulsive Interactions. Phys. Chem. Chem. Phys. 2019, 21, 4331-4338. https://doi.org/10.1039/c8cp07322h.

(3) Boys, S. F.; Bernardi, F. The Calculation of Small Molecular Interactions by the Differences of Separate Total Energies. Some Procedures with Reduced Errors. Mol. Phys. 1970, 19, 553-566. https://doi.org/10.1080/00268977000101561.

(4) Xantheas, S. S. On the Importance of the Fragment Relaxation Energy Terms in the Estimation of the Basis Set Superposition Error Correction to the Intermolecular Interaction Energy. J. Chem. Phys. 1996, 104, 8821-8824. https://doi.org/10.1063/1.471605.

(5) Loru, D.; Bermúdez, M. A.; Sanz, M. E. Structure of Fenchone by Broadband Rotational Spectroscopy. J. Chem. Phys. 2016, 145, 07114311-07114318. https://doi.org/10.1063/1.4961018.

(6) Loru, D.; Peña, I.; Sanz, M. E. Ethanol Dimer: Observation of Three New Conformers by Broadband Rotational Spectroscopy. J. Mol. Spectrosc. 2017, 335, 93-101. https://doi.org/10.1016/j.jms.2017.03.007.

(7) Kraitchman, J. Determination of Molecular Structure from Microwave Spectroscopic Data. Am. J. Phys. 1953, 21, 17-24. https://doi.org/10.1119/1.1933338.

(8) Costain, C. C. Determination of Molecular Structures from Ground State Rotational Constants. J. Chem. Phys. 1958, 29, 864-874. https://doi.org/10.1063/1.1744602. 
Table S1. Theoretical spectroscopic parameters and relative energies for the isomers of cyclooctanone- $\mathrm{H}_{2} \mathrm{O}$ within $900 \mathrm{~cm}^{-1}$.

\begin{tabular}{|c|c|c|c|c|c|c|c|c|}
\hline & \multicolumn{2}{|c|}{$1 w-I(B C 1)$} & \multicolumn{2}{|c|}{$1 w-I I(B C 1)$} & \multicolumn{2}{|c|}{$1 w-I I I(T B C 1)$} & \multicolumn{2}{|c|}{$1 w-I V(T B C 1)$} \\
\hline & B3LYP-D3BJa & MP2 & B3LYP-D3BJ & MP2 & B3LYP-D3BJ & MP2 & B3LYP-D3BJ & MP2 \\
\hline$A^{\mathrm{b}}(\mathrm{MHz})$ & 1409.3 & 1363.6 & 1763.9 & 1782.1 & 1879.0 & 1906.4 & 1507.0 & 1270.3 \\
\hline$B(\mathrm{MHz})$ & 1001.7 & 1062.2 & 891.3 & 887.3 & 856.6 & 850.0 & 881.6 & 1072.5 \\
\hline$C(\mathrm{MHz})$ & 842.3 & 900.9 & 719.3 & 720.6 & 668.2 & 668.8 & 728.3 & 963.6 \\
\hline$\mu_{a}(\mathrm{D})$ & -2.4 & -1.8 & 2.9 & 2.6 & 3.0 & 2.7 & 3.0 & 1.2 \\
\hline$\mu_{b}(\mathrm{D})$ & 0.3 & 0.2 & 0.6 & 0.6 & 0.7 & 0.7 & 0.4 & 0.2 \\
\hline$\mu_{c}(\mathrm{D})$ & -1.2 & -1.0 & -0.9 & -0.8 & -0.6 & -0.4 & 0.9 & 1.0 \\
\hline$\Delta E^{\mathrm{c}}\left(\mathrm{cm}^{-1}\right)$ & 74.4 & 0.0 & 0.0 & 52.3 & 609.7 & 768.1 & 783.2 & 811.2 \\
\hline$\Delta E_{0}^{\mathrm{d}}\left(\mathrm{cm}^{-1}\right)$ & 0.0 & 0.0 & 47.4 & 26.8 & 558.8 & 684.3 & 680.2 & 722.1 \\
\hline$D_{e}{ }^{\mathrm{e}}(\mathrm{kJ} / \mathrm{mol})$ & 32.5 & 22.8 & 33.4 & 23.6 & 34.3 & 23.8 & 32.3 & 21.8 \\
\hline & \multicolumn{2}{|c|}{$1 w-V(B C 2)$} & \multicolumn{2}{|c|}{$1 w-V I(B C 2)$} & \multicolumn{2}{|c|}{ 1w-VII (CR) } & & \\
\hline & B3LYP-D3BJ & MP2 & B3LYP-D3BJ & MP2 & B3LYP-D3BJ & MP2 & & \\
\hline$A^{\mathrm{b}}(\mathrm{MHz})$ & 1496.1 & 1418.3 & 1825.0 & 1841.2 & 1574.5 & 1578.1 & & \\
\hline$B(\mathrm{MHz})$ & 960.4 & 1023.5 & 892.4 & 887.0 & 933.0 & 934.2 & & \\
\hline$C(\mathrm{MHz})$ & 803.4 & 867.7 & 689.1 & 690.4 & 769.0 & 785.9 & & \\
\hline$\mu_{a}(\mathrm{D})$ & 2.3 & 1.8 & 2.9 & 2.7 & 2.4 & 2.2 & & \\
\hline$\mu_{b}(\mathrm{D})$ & 0.3 & 0.2 & 0.7 & 0.7 & 0.1 & 0.2 & & \\
\hline$\mu_{c}(\mathrm{D})$ & -1.1 & 0.8 & -0.6 & -0.4 & -1.2 & -1.0 & & \\
\hline$\Delta E^{\mathrm{c}}\left(\mathrm{cm}^{-1}\right)$ & 838.2 & 752.1 & 696.3 & 727.3 & 786.9 & 906.8 & & \\
\hline$\Delta E_{0}^{\mathrm{d}}\left(\mathrm{cm}^{-1}\right)$ & 866.5 & 760.4 & 778.0 & 796.9 & 748.6 & 837.7 & & \\
\hline$D_{e}{ }^{e}(\mathrm{~kJ} / \mathrm{mol})$ & 32.3 & 22.1 & 33.8 & 23.8 & 33.5 & 23.7 & & \\
\hline
\end{tabular}

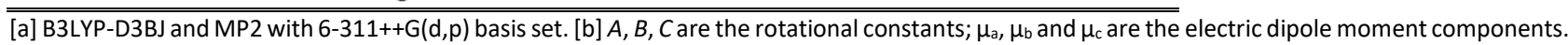
[c] Relative electronic energies. [d] Relative electronic energies including the zero-point correction [e] Binding energies from BSSE calculations including fragment relaxation. 
Table S2. Theoretical spectroscopic parameters and relative energies for the isomers of cyclooctanone- $\left(\mathrm{H}_{2} \mathrm{O}\right)_{2}$ within $800 \mathrm{~cm}^{-1}$.

\begin{tabular}{|c|c|c|c|c|c|c|c|c|}
\hline & \multicolumn{2}{|c|}{$2 w-I$ (BC1) } & \multicolumn{2}{|c|}{ 2w-II (BC1) } & \multicolumn{2}{|c|}{$2 \mathrm{w}-\mathrm{III}$ (BC1) } & \multicolumn{2}{|c|}{$2 w-I V(B C 1)$} \\
\hline & B3LYP-D3BJa & MP2 & B3LYP-D3BJ & MP2 & B3LYP-D3BJ & MP2 & B3LYP-D3BJ & MP2 \\
\hline$A^{\mathrm{b}}(\mathrm{MHz})$ & 1154.3 & 1163.9 & 1157.1 & 1157.1 & 1445.5 & 1445.3 & 1440.9 & 1446.1 \\
\hline$B(\mathrm{MHz})$ & 717.5 & 707.2 & 714.7 & 709.3 & 580.9 & 578.2 & 581.0 & 574.8 \\
\hline$C(\mathrm{MHz})$ & 581.8 & 575.0 & 578.3 & 577.9 & 522.8 & 521.9 & 508.6 & 503.9 \\
\hline$\mu_{a}(\mathrm{D})$ & 2.0 & 1.6 & 1.6 & 1.1 & 1.7 & 1.4 & 2.6 & 2.2 \\
\hline$\mu_{b}(\mathrm{D})$ & 0.0 & 0.2 & 0.2 & 0.4 & 0.9 & 0.9 & 0.1 & 0.0 \\
\hline$\mu_{c}(\mathrm{D})$ & -0.4 & -0.2 & -1.1 & -1.1 & -0.5 & -0.3 & 0.0 & 0.1 \\
\hline$\Delta E^{\mathrm{c}}\left(\mathrm{cm}^{-1}\right)$ & 0.0 & 0.0 & 112.1 & 103.8 & 143.8 & 199.9 & 142.8 & 201.9 \\
\hline$\Delta E_{0}^{\mathrm{d}}\left(\mathrm{cm}^{-1}\right)$ & 0.0 & 0.0 & 69.1 & 51.4 & 145.3 & 152.8 & 120.7 & 153.0 \\
\hline \multirow[t]{3}{*}{$D_{e}{ }^{\mathrm{e}}(\mathrm{kJ} / \mathrm{mol})$} & 72.5 & 53.0 & 71.3 & 52.0 & 71.2 & 51.8 & 71.5 & 52.1 \\
\hline & \multicolumn{2}{|c|}{$2 w-V(B C 1)$} & \multicolumn{2}{|c|}{ 2w-VI (BC1) } & \multicolumn{2}{|c|}{$2 w-V I I ~(B C 1)$} & \multicolumn{2}{|c|}{$2 w-V I I I(B C 1)$} \\
\hline & B3LYP-D3BJ & MP2 & B3LYP-D3BJ & MP2 & B3LYP-D3BJ & MP2 & B3LYP-D3BJ & MP2 \\
\hline$A^{\mathrm{b}}(\mathrm{MHz})$ & 1453.4 & 1460.7 & 1493.4 & 1490.0 & 1433.9 & 1458.4 & 1428.4 & 1471.6 \\
\hline$B(\mathrm{MHz})$ & 569.4 & 561.6 & 601.0 & 608.1 & 550.8 & 576.1 & 550.5 & 570.4 \\
\hline$C(\mathrm{MHz})$ & 513.5 & 512.7 & 521.8 & 526.3 & 497.3 & 533.2 & 490.6 & 529.6 \\
\hline$\mu_{a}(\mathrm{D})$ & 2.7 & 2.4 & -1.1 & -0.8 & -1.8 & -0.7 & -2.5 & -1.2 \\
\hline$\mu_{b}(\mathrm{D})$ & 0.4 & 0.4 & 0.1 & 0.3 & 0.4 & 0.6 & -0.4 & -1.0 \\
\hline$\mu_{c}(\mathrm{D})$ & 0.0 & 0.2 & 1.0 & 0.9 & 0.2 & 0.3 & -0.1 & -0.4 \\
\hline$\Delta E^{\mathrm{c}}\left(\mathrm{cm}^{-1}\right)$ & 87.6 & 155.8 & 371.7 & 222.9 & 315.2 & 303.6 & 408.9 & 320.1 \\
\hline$\Delta E_{0}^{\mathrm{d}}\left(\mathrm{cm}^{-1}\right)$ & 129.9 & 166.8 & 358.2 & 167.2 & 287.5 & 230.2 & 334.7 & 239.0 \\
\hline \multirow[t]{3}{*}{$D_{e}^{\mathrm{e}}(\mathrm{kJ} / \mathrm{mol})$} & 71.4 & 51.6 & 68.3 & 50.1 & 69.1 & 49.8 & 68.2 & 49.7 \\
\hline & \multicolumn{2}{|c|}{$2 w-I X(B C 1)$} & \multicolumn{2}{|c|}{$2 w-X(B C 1)$} & \multicolumn{2}{|c|}{$2 w-X I$ (TBC1) } & \multicolumn{2}{|c|}{$2 \mathrm{w}-\mathrm{XII}$ (BC1) } \\
\hline & B3LYP-D3BJ & MP2 & B3LYP-D3BJ & MP2 & B3LYP-D3BJ & MP2 & B3LYP-D3BJ & MP2 \\
\hline$A^{\mathrm{b}}(\mathrm{MHz})$ & 1279.3 & 1179.8 & 1176.7 & 1175.8 & 1091.4 & 1089.6 & 1054.3 & 1042.6 \\
\hline$B(\mathrm{MHz})$ & 631.0 & 712.4 & 706.1 & 707.6 & 738.5 & 738.2 & 762.4 & 783.8 \\
\hline$C(\mathrm{MHz})$ & 508.5 & 576.5 & 571.1 & 571.6 & 638.5 & 636.1 & 690.9 & 711.4 \\
\hline$\mu_{a}(\mathrm{D})$ & 2.5 & 1.5 & 2.5 & 2.1 & -1.2 & -0.8 & -3.1 & -3.0 \\
\hline$\mu_{b}(\mathrm{D})$ & -1.0 & -0.9 & -1.0 & -1.1 & -0.2 & -0.3 & 2.5 & 2.4 \\
\hline$\mu_{c}(\mathrm{D})$ & -1.4 & -0.7 & -1.4 & -1.1 & -0.2 & -0.1 & -2.4 & -2.0 \\
\hline$\Delta E^{\mathrm{c}}\left(\mathrm{cm}^{-1}\right)$ & 425.7 & 332.8 & 557.6 & 455.8 & 612.8 & 649.2 & 781.3 & 782.3 \\
\hline$\Delta E_{0}^{\mathrm{d}}\left(\mathrm{cm}^{-1}\right)$ & 390.9 & 280.7 & 443.3 & 319.3 & 576.6 & 606.2 & 805.7 & 663.7 \\
\hline$D_{e}^{e}(\mathrm{~kJ} / \mathrm{mol})$ & 67.7 & 49.1 & 66.2 & 48.6 & 64.9 & 54.5 & 63.3 & 43.3 \\
\hline
\end{tabular}

[a] B3LYP-D3BJ and MP2 with 6-311++G(d,p) basis set. [b] A, B, C are the rotational constants; $\mu_{a}, \mu_{b}$ and $\mu_{c}$ are the electric dipole moment components. [c] Relative electronic energies. [d] Relative electronic energies including the zero-point correction. [e] Binding energies from BSSE calculations including fragment relaxation. 
Table S3. Experimental spectroscopic parameters for all singly substituted ${ }^{13} \mathrm{C}$ and ${ }^{18} \mathrm{O}$ isotopic species of the observed cyclooctanone- $\left(\mathrm{H}_{2} \mathrm{O}\right)_{1,2}$ complexes.

\begin{tabular}{|c|c|c|c|c|c|}
\hline $1 w-I$ & $A^{\mathrm{a}}(\mathrm{MHz})$ & $B(\mathrm{MHz})$ & $C(\mathrm{MHz})$ & $\sigma^{c}(\mathrm{kHz})$ & $\mathrm{N}^{\mathrm{d}}$ \\
\hline${ }^{13} C_{1}$ & $1351.730(20)^{b}$ & $1025.4822(11)$ & $866.6115(11)$ & 6.7 & 10 \\
\hline${ }^{13} \mathrm{C}_{2}$ & $1357.308(19)$ & $1022.3081(11)$ & $870.0362(10)$ & 6.5 & 10 \\
\hline${ }^{13} C_{3}$ & $1363.400(15)$ & $1015.51781(83)$ & $866.65584(86)$ & 6.4 & 11 \\
\hline${ }^{13} \mathrm{C}_{4}$ & $1360.018(17)$ & $1018.1061(11)$ & $866.2339(12)$ & 6.5 & 8 \\
\hline${ }^{13} \mathrm{C}_{5}$ & $1356.9203(68)$ & $1022.88733(41)$ & $867.70985(43)$ & 3.0 & 7 \\
\hline${ }^{13} \mathrm{C}_{6}$ & $1361.6619(48)$ & $1024.90360(58)$ & $871.22396(57)$ & 5.5 & 12 \\
\hline${ }^{18} \mathrm{O}_{7}$ & $1348.98330(98)$ & $1019.49614(34)$ & $861.26116(35)$ & 4.4 & 19 \\
\hline${ }^{13} \mathrm{C}_{8}$ & $1353.028(13)$ & $1021.17404(86)$ & $870.37354(84)$ & 5.1 & 8 \\
\hline${ }^{13} \mathrm{C}_{9}$ & $1358.9177(97)$ & $1020.33585(58)$ & $872.25701(62)$ & 4.3 & 10 \\
\hline${ }^{18} \mathrm{O}_{10}$ & $1358.78082(50)$ & $984.93777(22)$ & $845.47419(24)$ & 2.9 & 21 \\
\hline \multicolumn{6}{|l|}{$1 w-I I$} \\
\hline${ }^{13} C_{1}$ & $1776.854(52)^{b}$ & $863.18511(89)$ & $702.14275(78)$ & 6.4 & 11 \\
\hline${ }^{13} \mathrm{C}_{2}$ & $1765.626(26)$ & $868.51283(75)$ & $705.15658(65)$ & 5.7 & 13 \\
\hline${ }^{13} C_{3}$ & $1756.309(32)$ & $871.97260(87)$ & $703.99941(76)$ & 6.7 & 14 \\
\hline${ }^{13} \mathrm{C}_{4}$ & $1762.599(25)$ & $872.12225(74)$ & $705.75516(65)$ & 5.7 & 15 \\
\hline${ }^{13} \mathrm{C}_{5}$ & $1770.146(18)$ & $870.18290(50)$ & $707.81121(43)$ & 3.8 & 13 \\
\hline${ }^{13} \mathrm{C}_{6}$ & $1772.808(33)$ & $872.77043(81)$ & $707.18568(72)$ & 6.2 & 12 \\
\hline${ }^{18} \mathrm{O}_{7}$ & $1754.5948(12)$ & $866.94279(40)$ & $702.02152(36)$ & 5.4 & 25 \\
\hline${ }^{13} \mathrm{C}_{8}$ & $1772.288(27)$ & $865.96086(74)$ & $703.05286(68)$ & 5.9 & 14 \\
\hline${ }^{13} \mathrm{C}_{9}$ & $1759.714(47)$ & $871.88704(99)$ & $704.56440(89)$ & 7.6 & 12 \\
\hline${ }^{18} \mathrm{O}_{10}$ & $1776.75883(70)$ & $833.936232(13)$ & $682.410557(28)$ & 6.3 & 42 \\
\hline \multicolumn{6}{|l|}{$2 w-I$} \\
\hline${ }^{13} \mathrm{C}_{1}$ & $1140.6978(87)^{b}$ & $697.93644(53)$ & $562.27452(40)$ & 5.4 & 14 \\
\hline${ }^{13} \mathrm{C}_{2}$ & $1144.055(13)$ & $695.99998(82)$ & $563.43629(51)$ & 6.1 & 12 \\
\hline${ }^{13} C_{3}$ & $1147.6805(11)$ & $691.84557(48)$ & $561.17383(49)$ & 6.4 & 14 \\
\hline${ }^{13} \mathrm{C}_{4}$ & $1148.488(13)$ & $691.79313(45)$ & $560.79547(48)$ & 5.9 & 16 \\
\hline${ }^{13} \mathrm{C}_{5}$ & $1146.309(11)$ & $694.43578(50)$ & $561.76922(50)$ & 7.3 & 18 \\
\hline${ }^{13} \mathrm{C}_{6}$ & $1147.1730(69)$ & $697.53459(37)$ & $564.05881(38)$ & 4.3 & 14 \\
\hline${ }^{18} \mathrm{O}_{7}$ & $1132.6442(66)$ & $697.78346(25)$ & $560.20857(25)$ & 3.4 & 20 \\
\hline${ }^{13} \mathrm{C}_{8}$ & $1141.850(14)$ & $695.91627(61)$ & $563.67886(45)$ & 4.5 & 11 \\
\hline${ }^{13} \mathrm{C}_{9}$ & $1146.0846(68)$ & $695.88273(41)$ & $564.86557(35)$ & 4.0 & 13 \\
\hline${ }^{18} \mathrm{O}_{10}$ & $1139.87404(31)$ & $679.93382(13)$ & $551.393886(14)$ & 3.4 & 44 \\
\hline${ }^{18} \mathrm{O}_{11}$ & $1141.81862(29)$ & $679.50480(14)$ & $550.61679(13)$ & 3.4 & 43 \\
\hline \multicolumn{6}{|l|}{$2 w-I V$} \\
\hline${ }^{13} \mathrm{C}_{1}$ & $1432.824(52)^{b}$ & $569.67561(68)$ & $498.40369(68)$ & 6.5 & 10 \\
\hline${ }^{13} \mathrm{C}_{2}$ & $1424.206(50)$ & $572.51492(56)$ & $500.74531(55)$ & 6.5 & 13 \\
\hline${ }^{13} \mathrm{C}_{3}$ & $1418.457(57)$ & $574.91369(82)$ & $500.55689(81)$ & 8.7 & 11 \\
\hline${ }^{13} \mathrm{C}_{4}$ & $1426.299(60)$ & $575.47057(73)$ & $501.84013(74)$ & 7.9 & 12 \\
\hline${ }^{13} C_{5}$ & $1429.776(60)$ & $574.79591(66)$ & $502.78323(57)$ & 6.4 & 14 \\
\hline${ }^{13} \mathrm{C}_{6}$ & $1429.067(66)$ & $575.4797(10)$ & $502.29030(87)$ & 8.7 & 12 \\
\hline${ }^{18} \mathrm{O}_{7}$ & $1409.301(29)$ & $574.95678(40)$ & 499.79099(41) & 5.1 & 18 \\
\hline${ }^{13} \mathrm{C}_{8}$ & $1430.314(40)$ & $570.64627(49)$ & $499.36085(50)$ & 6.2 & 13 \\
\hline${ }^{13} \mathrm{C}_{9}$ & $1422.777(53)$ & $573.66133(72)$ & $500.46046(61)$ & 8.2 & 12 \\
\hline${ }^{18} \mathrm{O}_{10}$ & $1417.688(16)$ & $561.73600(22)$ & $492.74634(21)$ & 3.3 & 32 \\
\hline${ }^{18} \mathrm{O}_{11}$ & $1420.3496(12)$ & $557.47670(16)$ & $488.72429(16)$ & 2.6 & 30 \\
\hline
\end{tabular}

[a] $A, B$ and $C$ are the rotational constants. $\Delta_{\mathrm{J}}, \Delta_{\mathrm{\jmath}}, \Delta_{\mathrm{k}}, \delta_{\mathrm{J}}$ and $\delta_{\mathrm{k}}$ centrifugal distortion constants were fixed to the values of the parent species. [b] Standard error in parentheses in units of the last digit. [c] $\sigma$ is the rms deviation of the fit. [d] $\mathrm{N}$ is the number of fitted transitions. 
Table S4. Substitution coordinates of the heavy atoms of the $1 \mathbf{w}-\mathbf{I}$ complex of cyclooctanone- $\mathrm{H}_{2} \mathrm{O}$ in $\AA$, and comparison with the MP2/6-311++G(d,p) values of possible matches (see text for full explanation).

\begin{tabular}{|c|c|c|c|c|c|c|c|c|c|}
\hline & \multicolumn{3}{|c|}{$r_{\mathrm{s}}$} & \multicolumn{6}{|c|}{$r_{\mathrm{e}}$} \\
\hline & \multicolumn{3}{|c|}{$1 w-1$} & \multicolumn{3}{|c|}{$1 w-I(B C 1)$} & \multicolumn{3}{|c|}{$1 w-V(B C 2)$} \\
\hline & $a$ & $\boldsymbol{b}$ & $c$ & $a$ & $\boldsymbol{b}$ & $c$ & $a$ & $b$ & $C$ \\
\hline $\mathrm{C}_{1}$ & $0.3946(39)^{a}$ & $-2.03577(75)$ & $-0.093(17)$ & 0.247 & 2.027 & 0.212 & 1.273 & -0.277 & 1.354 \\
\hline $\mathrm{C}_{2}$ & $0.7339(21)$ & $-1.1982(13)$ & $1.0860(14)$ & 0.664 & 1.243 & -1.040 & 1.995 & 0.947 & 0.778 \\
\hline $\mathrm{C}_{3}$ & $2.03925(75)$ & $-0.3070(50)$ & $0.9282(16)$ & 1.994 & 0.466 & -0.911 & 1.467 & 1.530 & -0.547 \\
\hline $\mathrm{C}_{4}$ & $1.78349(86)$ & $1.1655(13)$ & $0.7385(21)$ & 1.834 & -1.053 & -0.762 & -0.065 & 1.667 & -0.634 \\
\hline $\mathrm{C}_{5}$ & $1.0544(14)$ & $1.5615(10)$ & $-0.5459(28)$ & 1.124 & -1.529 & 0.521 & -0.792 & 0.525 & -1.381 \\
\hline $\mathrm{C}_{6}$ & $-0.3444(44)$ & $1.0387(15)$ & $-0.5770(26)$ & -0.328 & -1.092 & 0.548 & -0.633 & -0.838 & -0.746 \\
\hline $\mathrm{O}_{7}$ & $-1.2148(12)$ & $1.58860(95)$ & $0.115(13)$ & -1.145 & -1.662 & -0.170 & -1.549 & -1.374 & -0.128 \\
\hline $\mathrm{C}_{8}$ & $0.3417(44)$ & $-1.2696(12)$ & $-1.4759(10)$ & 0.223 & 1.223 & 1.516 & 1.374 & -1.580 & 0.552 \\
\hline $\mathrm{C}_{9}$ & $-0.7082(21)$ & $-0.074(20)$ & $-1.4706(10)$ & -0.732 & 0.024 & 1.488 & 0.714 & -1.530 & -0.842 \\
\hline $\mathrm{O}_{10}$ & $-3.04684(49)$ & $-0.4139(36)$ & $1.0225(15)$ & -2.957 & 0.371 & -1.028 & -2.867 & 0.806 & 1.181 \\
\hline
\end{tabular}

[a] Signs are taken from the theoretical calculations. Errors include Costain's error.

Table S5. Substitution coordinates of the heavy atoms of the $1 \mathbf{w}$-II complex of cyclooctanone- $\mathrm{H}_{2} \mathrm{O}$ in $\AA$, and comparison with the MP2/6-311++G(d,p) values of possible matches (see text for full explanation).

\begin{tabular}{cccc|ccc}
\hline \hline & \multicolumn{3}{c|}{$\boldsymbol{r}_{\mathrm{s}}$} & \multicolumn{3}{c}{$\boldsymbol{r}_{\mathrm{e}}$} \\
\hline \hline & \multicolumn{3}{c}{ 1w-II } & & \multicolumn{3}{c}{ 1w-II (BC1) } \\
& $\boldsymbol{a}$ & $\boldsymbol{b}$ & $\boldsymbol{c}$ & $\boldsymbol{a}$ & $\boldsymbol{b}$ & $\boldsymbol{c}$ \\
\hline $\mathrm{C}_{1}$ & $2.50917(61)^{\mathrm{a}}$ & $0.000(12)^{\mathrm{b}}$ & $0.6026(26)$ & -2.485 & 0.080 & -0.623 \\
$\mathrm{C}_{2}$ & $1.4264(11)$ & $1.0716(14)$ & $1.0102(15)$ & -1.386 & 1.078 & -1.018 \\
$\mathrm{C}_{3}$ & $0.8421(18)$ & $1.9155(8)$ & $-0.141(11)$ & -0.821 & 1.913 & 0.151 \\
$\mathrm{C}_{4}$ & $-0.5336(29)$ & $1.5151(10)$ & $-0.5970(26)$ & 0.583 & 1.498 & 0.610 \\
$\mathrm{C}_{5}$ & $-0.7094(21)$ & $0.000(19)^{\mathrm{b}}$ & $-1.1959(13)$ & 0.703 & 0.079 & 1.202 \\
$\mathrm{C}_{6}$ & $-0.4151(37)$ & $-0.9798(16)$ & $-0.1813(84)$ & 0.419 & -0.997 & 0.172 \\
$\mathrm{O}_{7}$ & $-1.2776(12)$ & $-1.2590(12)$ & $0.6579(22)$ & 1.253 & -1.271 & -0.686 \\
$\mathrm{C}_{8}$ & $2.12335(72)$ & $-0.9142(17)$ & $-0.5056(30)$ & -2.128 & -0.874 & 0.520 \\
$\mathrm{C}_{9}$ & $0.8540(18)$ & $-1.75220(88)$ & $-0.2423(64)$ & -0.897 & -1.742 & 0.243 \\
$\mathrm{O}_{10}$ & $-3.69017(41)$ & $0.2670(56)$ & $0.3484(43)$ & 3.651 & 0.254 & -0.356 \\
\hline \hline
\end{tabular}

[a] Signs are taken from the theoretical calculations. Errors include Costain's error. [b] Imaginary coordinate set to zero.

Table S6. Substitution coordinates of the heavy atoms of the $2 \mathbf{w}-\mathbf{I}$ complex of cyclooctanone- $\left(\mathrm{H}_{2} \mathrm{O}\right)_{2}$ in $\AA$, and comparison with the MP2/6-311++G(d,p) values of possible matches (see text for full explanation).

\begin{tabular}{|c|c|c|c|c|c|c|c|c|c|c|c|c|c|c|c|}
\hline \multicolumn{4}{|c|}{$r_{\mathrm{s}}$} & \multicolumn{12}{|c|}{$r_{\mathrm{e}}$} \\
\hline & \multicolumn{3}{|c|}{$2 w-I$} & \multicolumn{3}{|c|}{$2 w-I(B C 1)$} & \multicolumn{3}{|c|}{$2 w-I I(B C 1)$} & \multicolumn{3}{|c|}{$2 w-I X(B C 1)$} & \multicolumn{3}{|c|}{$2 w-X(B C 1)$} \\
\hline & $a$ & $\boldsymbol{b}$ & $c$ & $a$ & $b$ & $c$ & $a$ & $b$ & $c$ & $a$ & $b$ & $c$ & $a$ & $b$ & $c$ \\
\hline$C_{1}$ & $0.2423(72)^{\mathrm{a}}$ & $-2.03853(86)$ & -0.05 & 0.350 & -2.024 & 0.002 & 0.297 & -2.018 & -0.016 & -2.106 & 1.173 & -0.978 & -2.057 & 1.228 & -1.004 \\
\hline $\mathrm{C}_{2}$ & $0.8864(23)$ & $-1.2474(16)$ & 1.14 & 0.887 & -1.196 & 1.178 & 0.833 & -1.206 & 1.171 & -0.664 & 1.533 & -0.594 & -0.597 & 1.523 & -0.632 \\
\hline$C_{3}$ & 2.33 & -0.7 & 0 & 2.330 & -0.674 & 1.003 & 2.291 & -0.717 & 1.025 & -0.422 & 1.687 & 0.923 & -0.337 & 1.702 & 0.879 \\
\hline $\mathrm{C}_{4}$ & 2.4412 & 0.789 & 0. & 2.434 & 0.826 & 0.701 & 2.434 & 0.782 & 0.732 & 0.349 & 0.529 & 1.565 & 0.371 & 0.519 & 1.549 \\
\hline$C_{5}$ & $1.8332(10)$ & $1.2879(15)$ & $-0.58829(32)$ & 1.816 & 1.283 & -0.636 & 1.852 & 1.256 & -0.615 & -0.339 & -0.852 & 1.520 & -0.390 & -0.823 & 1.539 \\
\hline $\mathrm{C}_{6}$ & $0.3107(53)$ & 1.124 & -0.621 & 0.310 & 1.110 & -0.646 & 0.343 & 1.116 & -0.652 & -0.512 & -1.368 & 0.105 & -0.585 & -1.365 & 0.136 \\
\hline $\mathrm{O}_{7}$ & $-0.3271(48)$ & $1.92106(82)$ & $-0.032(50)$ & -0.388 & 1.906 & -0.021 & -0.348 & 1.923 & -0.033 & 0.444 & -1.831 & -0.513 & 0.348 & -1.887 & -0.468 \\
\hline $\mathrm{C}_{8}$ & $0.4611(45)$ & $-1.3173(16)$ & $-1.4059(15)$ & 0.451 & -1.353 & -1.371 & 0.440 & -1.347 & -1.385 & -2.689 & -0.056 & -0.274 & -2.696 & 0.043 & -0.273 \\
\hline$C_{9}$ & $-0.3279(51)$ & $0.0000(99)^{b}$ & $-1.4483(11)$ & -0.291 & -0.014 & -1.459 & -0.267 & 0.010 & -1.483 & -1.893 & -1.344 & -0.513 & -1.963 & -1.286 & -0.486 \\
\hline $\mathrm{O}_{10}$ & $-3.02262(50)$ & $1.3848(11)$ & $0.6874(22)$ & -3.035 & 1.366 & 0.728 & -2.966 & 1.375 & 0.815 & 3.163 & -1.149 & -0.322 & 3.095 & -1.225 & -0.491 \\
\hline $\mathrm{O}_{11}$ & $-3.12709(48)$ & $-1.3975(11)$ & $0.2214(68)$ & -3.059 & -1.385 & 0.168 & -3.094 & -1.351 & 0.119 & 2.836 & 1.664 & -0.585 & 2.982 & 1.610 & -0.430 \\
\hline
\end{tabular}

[a] Signs are taken from the theoretical calculations. Errors include Costain's error. [b] Imaginary coordinate set to zero. 
Table S7. Substitution coordinates of the heavy atoms of the $\mathbf{2} \mathbf{w}$-IV complex of cyclooctanone- $\left(\mathrm{H}_{2} \mathrm{O}\right)_{2}$ in $\AA$, and comparison with the MP2/6-311++G(d,p) values of possible matches (see text for full explanation).

\begin{tabular}{|c|c|c|c|c|c|c|c|c|c|}
\hline & \multirow{2}{*}{\multicolumn{3}{|c|}{$\frac{r_{\mathrm{s}}}{2 \mathrm{w}-\mathrm{IV}}$}} & \multicolumn{6}{|c|}{$r_{\mathrm{e}}$} \\
\hline & & & & \multicolumn{3}{|c|}{$2 w-I V(B C 1)$} & \multicolumn{3}{|c|}{$2 w-V(B C 1)$} \\
\hline & $a$ & $\boldsymbol{b}$ & $c$ & $a$ & $\boldsymbol{b}$ & $c$ & $a$ & $b$ & $c$ \\
\hline $\mathrm{C}_{1}$ & $2.98319(54)^{a}$ & $0.3138(52)$ & $0.4298(38)$ & 2.991 & 0.188 & 0.446 & 2.995 & 0.149 & 0.402 \\
\hline $\mathrm{C}_{2}$ & $1.79906(89)$ & $0.9953(16)$ & $1.2009(13)$ & 1.857 & 0.900 & 1.198 & 1.889 & 0.949 & 1.116 \\
\hline $\mathrm{C}_{3}$ & $0.9306(18)$ & $1.93830(85)$ & $0.3332(50)$ & 1.059 & 1.919 & 0.356 & 1.111 & 1.935 & 0.217 \\
\hline $\mathrm{C}_{4}$ & $-0.3649(45)$ & $1.3800(12)$ & $0.000(20)^{\mathrm{b}}$ & -0.340 & 1.445 & -0.057 & -0.303 & 1.479 & -0.154 \\
\hline $\mathrm{C}_{5}$ & $-0.3676(44)$ & $0.088(18)$ & $-1.0128(16)$ & -0.383 & 0.224 & -0.997 & -0.384 & 0.200 & -1.011 \\
\hline $\mathrm{C}_{6}$ & $0.135(13)$ & $-1.0552(16)$ & $-0.3093(55)$ & 0.132 & -1.029 & -0.323 & 0.109 & -1.015 & -0.255 \\
\hline $\mathrm{O}_{7}$ & $-0.4757(32)$ & $-1.69556(89)$ & $0.4920(31)$ & -0.552 & -1.624 & 0.508 & -0.571 & -1.514 & 0.641 \\
\hline $\mathrm{C}_{8}$ & $2.61765(60)$ & $-0.4368(36)$ & $-0.8479(19)$ & 2.590 & -0.492 & -0.866 & 2.568 & -0.599 & -0.862 \\
\hline $\mathrm{C}_{9}$ & $1.5691(10)$ & $-1.5330(11)$ & $-0.6630(25)$ & 1.498 & -1.557 & -0.709 & 1.448 & -1.617 & -0.634 \\
\hline $\mathrm{O}_{10}$ & $-3.09442(52)$ & $-0.8863(18)$ & $1.1499(14)$ & -3.221 & -0.950 & 1.079 & -3.072 & -0.491 & 1.401 \\
\hline $\mathrm{O}_{11}$ & $-3.68731(45)$ & $1.0200(17)$ & $-0.8635(20)$ & -3.620 & 1.056 & -0.847 & -3.781 & 0.576 & -1.104 \\
\hline
\end{tabular}

[a] Signs are taken from the theoretical calculations. Errors include Costain's error. [b] Imaginary coordinate set to zero. 
Table S8. B3LYP-D3BJ and MP2 spectroscopic parameters and relative energies for 1,1-cycloctanediol within $1000 \mathrm{~cm}^{-1}$.

\begin{tabular}{|c|c|c|c|c|c|c|c|c|}
\hline & \multicolumn{2}{|c|}{ I (BC5) } & \multicolumn{2}{|c|}{ II (BC5) } & \multicolumn{2}{|c|}{ III (BC3) } & \multicolumn{2}{|c|}{ IV (TBC3) } \\
\hline & B3LYP-D3BJe & MP2 & B3LYP-D3BJ & MP2 & B3LYP-D3BJ & MP2 & B3LYP-D3BJ & MP2 \\
\hline$A^{\mathrm{a}}(\mathrm{MHz})$ & 1848.8 & 1855.2 & 1854.2 & 1862.9 & 1909.4 & 1915.2 & 1916.5 & 1942.0 \\
\hline$B(\mathrm{MHz})$ & 1115.5 & 1129.3 & 1110.0 & 1121.9 & 1062.3 & 1073.9 & 1050.1 & 1054.1 \\
\hline$C(\mathrm{MHz})$ & 850.3 & 859.3 & 854.3 & 862.8 & 868.3 & 878.7 & 834.8 & 842.2 \\
\hline$\mu_{a}(\mathrm{D})$ & 0.5 & 0.5 & 0.5 & 0.4 & -0.5 & -0.4 & 0.5 & 0.4 \\
\hline$\mu_{b}$ (D) & 0.0 & 0.0 & 0.1 & 0.1 & 0.0 & 0.1 & -0.1 & -0.1 \\
\hline$\mu_{c}(\mathrm{D})$ & -0.1 & 0.1 & 0.0 & 0.0 & 0.1 & 0.1 & 0.0 & 0.0 \\
\hline$\Delta E^{\mathrm{b}}\left(\mathrm{cm}^{-1}\right)$ & 0.0 & 0.0 & 6.0 & 52.0 & 85.7 & 100.4 & 541.1 & 580.8 \\
\hline$\Delta E_{0}{ }^{c}\left(\mathrm{~cm}^{-1}\right)$ & 0.0 & 0.0 & 1.5 & 56.0 & 30.5 & 99.9 & 488.8 & 527.0 \\
\hline \multirow[t]{3}{*}{$\Delta G^{\mathrm{d}}\left(\mathrm{cm}^{-1}\right)$} & 0.0 & 0.0 & 10.8 & 69.4 & 40.6 & 119.6 & 428.4 & 462.9 \\
\hline & \multicolumn{2}{|c|}{ V (TBC3) } & \multicolumn{2}{|c|}{ VI (BC2) } & \multicolumn{2}{|c|}{ VII (BC2) } & \multicolumn{2}{|c|}{ VIII (TBC2) } \\
\hline & B3LYP-D3BJe & MP2 & B3LYP-D3BJ & MP2 & B3LYP-D3BJ & MP2 & B3LYP-D3BJ & MP2 \\
\hline$A^{\mathrm{a}}(\mathrm{MHz})$ & 1910.2 & 1934.9 & 1886.9 & 1898.1 & 1892.3 & 1904.8 & 1912.5 & 1996.6 \\
\hline$B(\mathrm{MHz})$ & 1054.3 & 1059.0 & 1121.9 & 1129.3 & 1121.2 & 1128.2 & 1036.9 & 1023.3 \\
\hline$C(\mathrm{MHz})$ & 831.3 & 838.8 & 885.8 & 893.5 & 882.1 & 889.1 & 812.2 & 822.0 \\
\hline$\mu_{a}(\mathrm{D})$ & 0.6 & -0.6 & 0.4 & 0.4 & 0.3 & -0.3 & 0.5 & -0.4 \\
\hline$\mu_{b}(\mathrm{D})$ & -0.1 & -0.1 & -0.2 & -0.2 & 0.0 & 0.0 & -0.1 & 0.0 \\
\hline$\mu_{c}(\mathrm{D})$ & 0.1 & 0.1 & 0.0 & -0.2 & 0.0 & 0.0 & 0.0 & 0.1 \\
\hline$\Delta E^{\mathrm{b}}\left(\mathrm{cm}^{-1}\right)$ & 586.0 & 603.7 & 672.8 & 840.6 & 718.1 & 855.1 & 730.8 & 1021.3 \\
\hline$\Delta E_{0}{ }^{\mathrm{c}}\left(\mathrm{cm}^{-1}\right)$ & 524.3 & 533.3 & 683.9 & 842.6 & 732.6 & 846.3 & 552.6 & 873.1 \\
\hline \multirow[t]{3}{*}{$\Delta G^{\mathrm{d}}\left(\mathrm{cm}^{-1}\right)$} & 449.9 & 455.0 & 726.2 & 853.5 & 775.6 & 846.3 & 183.0 & 673.3 \\
\hline & \multicolumn{2}{|c|}{ IX (BC5) } & \multicolumn{2}{|c|}{ X (BB2) } & \multicolumn{2}{|c|}{ XI (BC1) } & \multicolumn{2}{|c|}{ XII (BC1) } \\
\hline & B3LYP-D3BJe & MP2 & B3LYP-D3BJ & MP2 & B3LYP-D3BJ & MP2 & B3LYP-D3BJ & MP2 \\
\hline$A^{\mathrm{a}}(\mathrm{MHz})$ & 1844.2 & 1849.5 & 1843.3 & 1855.7 & 1810.1 & 1824.2 & 1809.6 & 1823.8 \\
\hline$B(\mathrm{MHz})$ & 1115.1 & 1128.8 & 1138.5 & 1160.8 & 1153.4 & 1156.5 & 1152.9 & 1156.0 \\
\hline$C(\mathrm{MHz})$ & 856.1 & 864.8 & 889.6 & 911.5 & 908.2 & 912.8 & 910.3 & 915.8 \\
\hline$\mu_{a}(\mathrm{D})$ & 0.7 & -0.6 & 0.5 & -0.5 & 0.3 & -0.2 & 0.3 & -0.3 \\
\hline$\mu_{b}(\mathrm{D})$ & 1.6 & -1.6 & 0.0 & 0.0 & -0.1 & -0.1 & 0.1 & 0.1 \\
\hline$\mu_{c}(\mathrm{D})$ & -1.8 & 1.8 & 0.1 & 0.1 & 0.0 & 0.0 & 0.1 & 0.1 \\
\hline$\Delta E^{\mathrm{b}}\left(\mathrm{cm}^{-1}\right)$ & 974.0 & 1026.5 & 957.0 & 1056.4 & 922.1 & 1139.8 & 936.4 & 1219.1 \\
\hline$\Delta E_{0}{ }^{c}\left(\mathrm{~cm}^{-1}\right)$ & 835.5 & 922.7 & 1050.0 & 1124.6 & 911.3 & 1122.0 & 921.1 & 1185.4 \\
\hline$\Delta G^{\mathrm{d}}\left(\mathrm{cm}^{-1}\right)$ & 705.2 & 836.2 & 997.3 & 1051.9 & 924.4 & 1104.0 & 939.1 & 1162.3 \\
\hline
\end{tabular}

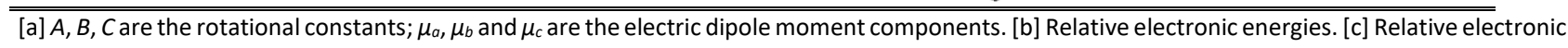
energies including the zero-point correction. [d] Gibbs free energy at $346 \mathrm{~K}$. [e] B3LYP-D3BJ and MP2 with 6-311++G(d,p) basis set. 
Table S9. Experimental and theoretical bond lengths (in $\AA$ ) and angles (in degrees) of cyclooctanone- $\mathrm{H}_{2} \mathrm{O}$ and comparison with the experimental structural parameters of bare cyclooctanone.

\begin{tabular}{|c|c|c|c|c|c|c|c|}
\hline & \multicolumn{3}{|c|}{$1 w-I$} & \multicolumn{3}{|c|}{$1 w-I I$} & \multirow{2}{*}{$\begin{array}{r}\text { BC1 } \\
r_{0}\end{array}$} \\
\hline & $r_{s}^{a}$ & $r_{0}^{b}$ & $r_{e}^{c}$ & $r_{s}^{a}$ & $r_{0}^{b}$ & $r_{e}^{c}$ & \\
\hline$r\left(C_{2}-C_{1}\right)$ & $1.485(18)$ & $1.537(17)$ & 1.536 & $1.577(14)$ & $1.540(12)$ & 1.536 & $1.539(6)$ \\
\hline$r\left(\mathrm{C}_{3}-\mathrm{C}_{2}\right)$ & $1.588(4)$ & $1.551(18)$ & 1.545 & $1.542(11)$ & $1.543(11)$ & 1.544 & $1.544(6)$ \\
\hline$r\left(\mathrm{C}_{4}-\mathrm{C}_{3}\right)$ & $1.507(6)$ & $1.545(18)$ & 1.535 & $1.504(6)$ & $1.534(14)$ & 1.534 & $1.540(6)$ \\
\hline$r\left(C_{5}-C_{4}\right)$ & $1.529(3)$ & $1.501(14)$ & 1.542 & $1.639(20)$ & $1.565(11)$ & 1.543 & $1.541(6)$ \\
\hline$r\left(\mathrm{C}_{6}-\mathrm{C}_{5}\right)$ & $1.494(4)$ & - & 1.517 & $1.441(17)$ & - & 1.517 & $1.520(9)^{d}$ \\
\hline$r\left(\mathrm{C}_{6}-\mathrm{O}_{7}\right)$ & $1.240(8)$ & $1.209(17)$ & 1.228 & $1.235(9)$ & $1.211(16)$ & 1.228 & $1.215(9)$ \\
\hline$r\left(\mathrm{C}_{9}-\mathrm{C}_{6}\right)$ & $1.473(17)$ & $1.523(18)$ & 1.514 & $1.487(5)$ & $1.534(18)$ & 1.515 & $1.520(9)$ \\
\hline$r\left(\mathrm{C}_{9}-\mathrm{C}_{8}\right)$ & $1.591(16)$ & - & 1.533 & $1.544(3)$ & $1.539(12)$ & 1.533 & $1.535(6)$ \\
\hline$r\left(\mathrm{C}_{8}-\mathrm{C}_{1}\right)$ & $1.582(20)$ & $1.555(14)$ & 1.532 & $1.488(13)$ & $1.526(10)$ & 1.531 & $1.533(6)$ \\
\hline$r\left(\mathrm{O}_{7}-\mathrm{O}_{10}\right)$ & $2.862(5)$ & $2.841(7)$ & 2.857 & $2.871(3)$ & $2.871(6)$ & 2.863 & - \\
\hline$\angle\left(C_{3}-C_{2}-C_{1}\right)$ & $115.2(5)$ & - & 115.0 & $116.0(4)$ & $115.6(6)$ & 114.7 & $115.2(3)$ \\
\hline$\angle\left(C_{4}-C_{3}-C_{2}\right)$ & $114.9(2)$ & $114.6(5)$ & 114.6 & $115.3(4)$ & $114.3(3)$ & 114.5 & $114.8(2)$ \\
\hline$\angle\left(C_{5}-C_{4}-C_{3}\right)$ & $116.1(2)$ & $116.2(7)$ & 115.7 & $117.1(3)$ & $116.7(7)$ & 115.8 & $115.7(2)$ \\
\hline$\angle\left(\mathrm{C}_{6}-\mathrm{C}_{5}-\mathrm{C}_{4}\right)$ & $111.9(2)$ & - & 111.5 & $110.5(5)$ & - & 112.2 & $111.8(4)^{d}$ \\
\hline$\angle\left(\mathrm{O}_{7}-\mathrm{C}_{6}-\mathrm{C}_{5}\right)$ & $119.4(3)$ & - & 119.5 & 119.3(4) & - & 120.5 & $120.4(5)^{d}$ \\
\hline$\angle\left(\mathrm{O}_{7}-\mathrm{C}_{6}-\mathrm{C}_{9}\right)$ & $120.0(4)$ & - & 121.8 & $120.4(6)$ & $120.6(8)$ & 120.8 & $118.8(6)^{d}$ \\
\hline$\angle\left(\mathrm{C}_{9}-\mathrm{C}_{6}-\mathrm{C}_{5}\right)$ & $120.6(3)$ & - & 118.6 & $119.9(7)$ & - & 118.7 & $120.7(8)$ \\
\hline$\angle\left(\mathrm{C}_{8}-\mathrm{C}_{9}-\mathrm{C}_{6}\right)$ & $114.0(2)$ & $115.7(5)$ & 114.9 & $115.3(2)$ & $115.8(5)$ & 115.2 & $115.6(3)$ \\
\hline$\angle\left(\mathrm{C}_{9}-\mathrm{C}_{8}-\mathrm{C}_{1}\right)$ & $112.5(4)$ & $113.6(5)$ & 113.9 & $114.8(5)$ & $114.9(3)$ & 114.0 & $114.5(4)$ \\
\hline$\angle\left(C_{8}-C_{1}-C_{2}\right)$ & $115.4(2)$ & $115.7(5)$ & 115.5 & $115.6(2)$ & $115.1(3)$ & 115.5 & $116.0(2)$ \\
\hline$\tau\left(C_{4} C_{3} C_{2} C_{1}\right)$ & $106.5(4)$ & - & 105.6 & $102.9(8)$ & - & 106.0 & $105.1(2)$ \\
\hline$\tau\left(C_{5} C_{4} C_{3} C_{2}\right)$ & $-64.7(2)$ & - & -64.5 & $-61.3(9)$ & - & -64.0 & - \\
\hline$\tau\left(C_{6} C_{5} C_{4} C_{3}\right)$ & $65.6(2)$ & - & 65.8 & $63.0(7)$ & - & 65.4 & - \\
\hline$\tau\left(\mathrm{O}_{7} \mathrm{C}_{6} \mathrm{C}_{5} \mathrm{C}_{4}\right)$ & $75.5(6)$ & - & 74.4 & $80.5(6)$ & - & 75.6 & - \\
\hline$\tau\left(C_{9} C_{6} C_{5} C_{4}\right)$ & $-107.6(7)$ & - & -106.8 & $-106.4(7)$ & - & -105.9 & - \\
\hline$\tau\left(C_{8} C_{9} C_{6} C_{5}\right)$ & $44.4(9)$ & - & 44.0 & $47.0(12)$ & - & 43.0 & - \\
\hline$\tau\left(\mathrm{C}_{8} \mathrm{C}_{9} \mathrm{C}_{6} \mathrm{O}_{7}\right)$ & $-138.7(6)$ & - & -137.3 & $-139.9(6)$ & - & -138.5 & $-139.7(4)$ \\
\hline$\tau\left(C_{1} C_{8} C_{9} C_{6}\right)$ & 67.1(9) & - & 67.6 & $68.0(10)$ & - & 67.8 & $67.4(10)$ \\
\hline$\tau\left(C_{2} C_{1} C_{8} C_{9}\right)$ & $-58.2(7)$ & - & -59.5 & $-59.0(11)$ & - & -58.8 & $-57.4(5)$ \\
\hline$\tau\left(C_{3} C_{2} C_{1} C_{8}\right)$ & $-51.1(5)$ & - & -52.0 & $-51.2(10)$ & - & -53.0 & $-53.6(5)$ \\
\hline
\end{tabular}

[a] The substitution structure has been determined from the atomic coordinates including Costain's error, and with signs taken from $a b$ initio calculations. [b] Effective structure; non fitted parameters were fixed to the MP2/6-311++G(d,p) values. [c] MP2/6-311++G(d,p) values. [d] Derived from the determined $r_{0}$ structure, not fitted directly. 
Table S10. Experimental and theoretical bond lengths (in $\AA$ ) and angles (in degrees) of cyclooctanone- $\left(\mathrm{H}_{2} \mathrm{O}\right)_{2}$ and comparison with the experimental structural parameters of bare cyclooctanone.

\begin{tabular}{|c|c|c|c|c|c|c|c|}
\hline & \multicolumn{3}{|c|}{$2 w-I$} & \multicolumn{3}{|c|}{$2 w-I V$} & \multirow{2}{*}{$\begin{array}{c}\text { BC1 } \\
r_{0}^{b}\end{array}$} \\
\hline & $r_{s}^{a}$ & $r_{0}^{b}$ & $r_{e}^{c}$ & $r_{s}$ & $r_{0}^{b}$ & $r_{e}^{c}$ & \\
\hline$r\left(\mathrm{C}_{2}-\mathrm{C}_{1}\right)$ & $1.571(26)$ & $1.519(24)$ & 1.535 & $1.569(7)$ & $1.529(35)$ & 1.536 & $1.539(6)$ \\
\hline$r\left(C_{3}-C_{2}\right)$ & $1.558(2)$ & $1.561(15)$ & 1.545 & $1.548(8)$ & $1.574(45)$ & 1.545 & $1.544(6)$ \\
\hline$r\left(\mathrm{C}_{4}-\mathrm{C}_{3}\right)$ & $1.520(4)$ & $1.553(17)$ & 1.534 & $1.449(16)$ & $1.567(44)$ & 1.534 & $1.540(6)$ \\
\hline$r\left(\mathrm{C}_{5}-\mathrm{C}_{4}\right)$ & $1.539(4)$ & $1.508(18)$ & 1.542 & $1.642(48)$ & $1.569(24)$ & 1.542 & $1.541(6)$ \\
\hline$r\left(\mathrm{C}_{6}-\mathrm{C}_{5}\right)$ & $1.532(5)$ & $1.524(19)$ & 1.516 & $1.433(39)$ & $1.502(55)$ & 1.513 & $1.520(9)^{d}$ \\
\hline$r\left(\mathrm{C}_{6}-\mathrm{O}_{7}\right)$ & $1.179(25)$ & $1.224(19)$ & 1.230 & $1.194(20)$ & $1.206(48)$ & 1.230 & $1.215(9)$ \\
\hline$r\left(\mathrm{C}_{9}-\mathrm{C}_{6}\right)$ & $1.534(8)$ & - & 1.512 & $1.553(30)$ & - & 1.514 & $1.520(9)$ \\
\hline$r\left(\mathrm{C}_{9}-\mathrm{C}_{8}\right)$ & $1.536(9)$ & $1.542(26)$ & 1.534 & $1.528(5)$ & $1.546(48)$ & 1.533 & $1.535(6)$ \\
\hline$r\left(\mathrm{C}_{8}-\mathrm{C}_{1}\right)$ & $1.549(29)$ & $1.550(23)$ & 1.532 & $1.526(10)$ & - & 1.531 & $1.533(6)$ \\
\hline$r\left(\mathrm{O}_{7}-\mathrm{O}_{10}\right)$ & $2.841(13)$ & $2.792(12)$ & 2.803 & $2.819(4)$ & $2.797(34)$ & 2.812 & - \\
\hline$r\left(\mathrm{O}_{10}-\mathrm{O}_{11}\right)$ & $2.823(2)$ & $2.831(9)$ & 2.808 & $2.835(2)$ & $2.813(23)$ & 2.810 & - \\
\hline$\angle\left(\mathrm{C}_{3}-\mathrm{C}_{2}-\mathrm{C}_{1}\right)$ & $118.9(7)$ & - & 115.0 & $114.4(5)$ & - & 114.9 & $115.2(3)$ \\
\hline$\angle\left(\mathrm{C}_{4}-\mathrm{C}_{3}-\mathrm{C}_{2}\right)$ & $114.9(1)$ & $114.8(6)$ & 114.6 & $113.3(11)$ & $114.4(16)$ & 114.5 & $114.8(2)$ \\
\hline$\angle\left(C_{5}-C_{4}-C_{3}\right)$ & $115.8(2)$ & $115.2(5)$ & 115.6 & $116.5(14)$ & - & 115.7 & $115.7(2)$ \\
\hline$\angle\left(C_{6}-C_{5}-C_{4}\right)$ & $112.1(2)$ & $114.2(8)$ & 111.7 & $108.9(14)$ & $111.7(16)$ & 112.0 & $111.8(4)^{d}$ \\
\hline$\angle\left(\mathrm{O}_{7}-\mathrm{C}_{6}-\mathrm{C}_{5}\right)$ & $117.0(7)$ & - & 119.1 & $125.3(24)$ & - & 120.8 & $120.4(5)^{d}$ \\
\hline$\angle\left(\mathrm{O}_{7}-\mathrm{C}_{6}-\mathrm{C}_{9}\right)$ & $122.7(9)$ & - & 121.9 & $117.4(12)$ & - & 120.4 & $118.8(6)^{d}$ \\
\hline$\angle\left(\mathrm{C}_{9}-\mathrm{C}_{6}-\mathrm{C}_{5}\right)$ & $120.2(3)$ & - & 118.9 & $117.2(14)$ & - & 118.8 & $120.7(8)$ \\
\hline$\angle\left(\mathrm{C}_{8}-\mathrm{C}_{9}-\mathrm{C}_{6}\right)$ & $113.6(3)$ & - & 115.2 & $116.2(5)$ & - & 115.2 & $115.6(3)$ \\
\hline$\angle\left(\mathrm{C}_{9}-\mathrm{C}_{8}-\mathrm{C}_{1}\right)$ & $110.5(4)$ & $112.8(7)$ & 113.7 & $114.6(4)$ & $118.6(25)$ & 114.0 & $114.5(4)$ \\
\hline$\angle\left(\mathrm{C}_{8}-\mathrm{C}_{1}-\mathrm{C}_{2}\right)$ & $111.8(3)$ & $115.8(6)$ & 115.3 & $116.4(2)$ & $116.2(12)$ & 115.6 & $116.0(2)$ \\
\hline$\angle\left(\mathrm{O}_{10}-\mathrm{O}_{7}-\mathrm{C}_{6}\right)$ & $120.8(5)$ & $124.2(4)$ & & $118.5(12)$ & $119.1(12)$ & & - \\
\hline$\angle\left(\mathrm{O}_{7}-\mathrm{O}_{10}-\mathrm{O}_{11}\right)$ & $100.3(2)$ & $100.1(2)$ & 98.3 & $102.80(1)$ & $105.9(5)$ & 99.6 & - \\
\hline$\tau\left(\mathrm{C}_{4} \mathrm{C}_{3} \mathrm{C}_{2} \mathrm{C}_{1}\right)$ & $104.0(6)$ & - & 105.8 & 106.0(19) & $105.6(18)$ & 105.7 & - \\
\hline$\tau\left(\mathrm{C}_{5} \mathrm{C}_{4} \mathrm{C}_{3} \mathrm{C}_{2}\right)$ & $-64.3(2)$ & - & -64.2 & $-67.9(33)$ & - & -64.3 & - \\
\hline$\tau\left(C_{6} C_{5} C_{4} C_{3}\right)$ & $66.5(2)$ & - & 66.2 & $71.8(32)$ & - & 66.2 & - \\
\hline$\tau\left(\mathrm{O}_{7} \mathrm{C}_{6} \mathrm{C}_{5} \mathrm{C}_{4}\right)$ & $78.2(22)$ & - & 75.6 & $76.6(23)$ & - & 75.1 & - \\
\hline$\tau\left(\mathrm{C}_{9} \mathrm{C}_{6} \mathrm{C}_{5} \mathrm{C}_{4}\right)$ & $-104.5(4)$ & - & -106.2 & $-106.1(11)$ & - & -106.2 & - \\
\hline$\tau\left(C_{8} C_{9} C_{6} C_{5}\right)$ & $38.4(4)$ & - & 42.1 & 42.1(19) & - & 42.5 & - \\
\hline$\tau\left(\mathrm{C}_{8} \mathrm{C}_{9} \mathrm{C}_{6} \mathrm{O}_{7}\right)$ & $-144.5(23)$ & - & -139.7 & $-140.4(10)$ & - & -138.7 & $-139.7(4)$ \\
\hline$\tau\left(C_{1} C_{8} C_{9} C_{6}\right)$ & $75.8(7)$ & - & 68.7 & $68.1(9)$ & - & 68.0 & $67.4(10)$ \\
\hline$\tau\left(C_{2} C_{1} C_{8} C_{9}\right)$ & $-67.6(5)$ & - & -58.8 & $-56.4(9)$ & $-55.2(23)$ & -58.3 & $-57.4(5)$ \\
\hline$\tau\left(C_{3} C_{2} C_{1} C_{8}\right)$ & $-46.5(4)$ & - & -53.1 & $-53.3(8)$ & - & -53.1 & $-53.6(5)$ \\
\hline$\tau\left(\mathrm{C}_{6} \mathrm{O}_{7} \mathrm{O}_{10} \mathrm{O}_{11}\right)$ & $13.7(31)$ & - & 12.3 & $12.5(9)$ & - & 4.6 & - \\
\hline
\end{tabular}

[a] The substitution structure has been determined from the atomic coordinates including Costain's error, and with signs taken from ab initio calculations. [b] Effective structure; non fitted parameters were fixed to the MP2/6-311++G(d,p) values. [c] MP2/6-311++G(d,p) values. [d] Derived from the determined $r_{0}$ structure, not fitted directly. 
Table S11. Binding energy decomposition analysis in $\mathrm{kJ} \mathrm{mol}^{-1}$ for the complexes of cyclooctanone- $\mathrm{H}_{2} \mathrm{O}$ on their MP2 and B3LYP-D3BJ geometries, using SAPT(0)/jun-cc-pDVZ calculations within Psi4. The overall binding energies are in agreement with those obtained from B3LYP-D3BJ and MP2 calculations.

\begin{tabular}{|c|c|c|c|c|c|c|c|c|c|c|}
\hline & \multicolumn{2}{|c|}{$\Delta \mathrm{E}_{\text {electrostatic }}$} & \multicolumn{2}{|c|}{$\Delta \mathrm{E}_{\text {exchange }}$} & \multicolumn{2}{|c|}{$\Delta \mathrm{E}_{\text {induction }}$} & \multicolumn{2}{|c|}{$\Delta \mathrm{E}_{\text {dispersion }}$} & \multicolumn{2}{|c|}{$\Delta \mathrm{E}_{\text {total }}$} \\
\hline & MP2 & B3LYP & MP2 & B3LYP & MP2 & B3LYP & MP2 & B3LYP & MP2 & B3LYP \\
\hline $1 w-1$ & -46.5 & -51.3 & 40.3 & 46.7 & -12.8 & -15.1 & -12.8 & -12.6 & -31.8 & -32.2 \\
\hline $1 w-I I$ & -49.0 & -53.1 & 42.1 & 49.0 & -13.5 & -15.8 & -11.9 & -12.8 & -32.4 & -32.7 \\
\hline $1 w-I I I$ & -49.5 & -53.7 & 42.3 & 49.3 & -13.7 & -15.9 & -11.9 & -12.9 & -32.8 & -33.3 \\
\hline $1 w-I V$ & -44.1 & -51.8 & 37.8 & 46.8 & -11.7 & -15.2 & -12.9 & -11.5 & -30.8 & -31.9 \\
\hline $1 w-V$ & -44.5 & -50.3 & 38.9 & 45.4 & -12.2 & -14.7 & -13.3 & -12.4 & -31.0 & -32.0 \\
\hline $1 w-V I$ & -49.5 & -53.5 & 42.6 & 49.4 & -13.8 & -16.0 & -12.1 & -13.0 & -32.8 & -33.0 \\
\hline $1 w-V I I$ & -48.4 & -52.6 & 41.4 & 48.3 & -13.7 & -15.7 & -12.0 & -12.9 & -32.5 & -32.8 \\
\hline
\end{tabular}


Table S12. B3LYP-D3BJ and MP2 spectroscopic parameters and relative energies for the 1,1-cycloctanediol$\mathrm{H}_{2} \mathrm{O}$ complex within $1000 \mathrm{~cm}^{-1}$.

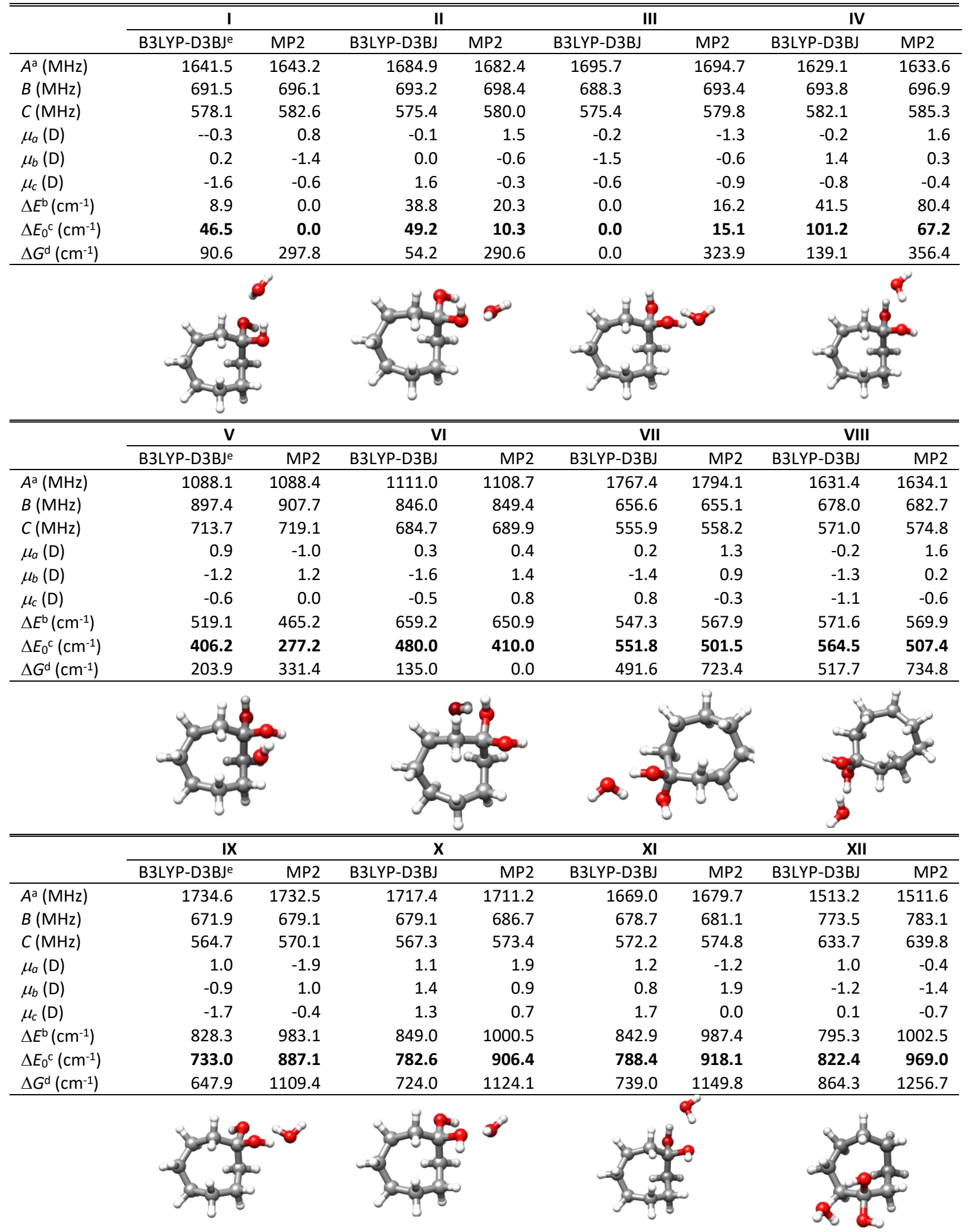

[a] $A, B, C$ are the rotational constants; $\mu_{a}, \mu_{b}$ and $\mu_{c}$ are the electric dipole moment components. [b] Relative electronic energies. [c] Relative electronic energies including the zero-point correction. [d] Gibbs free energy at $346 \mathrm{~K}$. [e] B3LYP-D3BJ and MP2 with 6-311++G(d,p) basis set. 
Table S12 (cont.). B3LYP-D3BJ and MP2 spectroscopic parameters and relative energies for the 1,1cycloctanediol- $\mathrm{H}_{2} \mathrm{O}$ complex within $1000 \mathrm{~cm}^{-1}$.

\begin{tabular}{|c|c|c|c|c|c|c|}
\hline & \multicolumn{2}{|c|}{ XIII } & \multicolumn{2}{|c|}{ XIV } & \multicolumn{2}{|l|}{$x V$} \\
\hline & B3LYP-D3BJe & MP2 & B3LYP-D3BJ & MP2 & B3LYP-D3BJ & MP2 \\
\hline$A^{\mathrm{a}}(\mathrm{MHz})$ & 1465.2 & 1467.4 & 1476.0 & 1480.0 & 1505.9 & 1512.8 \\
\hline$B(\mathrm{MHz})$ & 750.7 & 755.2 & 737.9 & 739.2 & 774.6 & 779.3 \\
\hline$C(\mathrm{MHz})$ & 643.5 & 646.3 & 639.0 & 640.4 & 631.0 & 633.1 \\
\hline$\mu_{a}(\mathrm{D})$ & 1.0 & -0.4 & -0.1 & 0.4 & -0.3 & 1.2 \\
\hline$\mu_{b}$ (D) & 1.1 & -1.1 & 0.5 & 0.9 & 0.3 & -0.5 \\
\hline$\mu_{c}(\mathrm{D})$ & 0.4 & 1.2 & 1.6 & 1.3 & -1.7 & 1.3 \\
\hline$\Delta E^{\mathrm{b}}\left(\mathrm{cm}^{-1}\right)$ & 826.8 & 987.5 & 810.7 & 1011.8 & 925.1 & 1095.1 \\
\hline$\Delta E_{0} \mathrm{c}\left(\mathrm{cm}^{-1}\right)$ & 885.6 & 972.5 & 886.2 & 998.0 & 941.3 & 1061.4 \\
\hline$\Delta G^{\mathrm{d}}\left(\mathrm{cm}^{-1}\right)$ & 941.3 & 1257.6 & 941.1 & 1259.8 & 962.2 & 1330.0 \\
\hline
\end{tabular}

[a] $A, B, C$ are the rotational constants; $\mu_{a}, \mu_{b}$ and $\mu_{c}$ are the electric dipole moment components. [b] Relative electronic energies. [c] Relative electronic energies including the zero-point correction. [d] Gibbs free energy at $346 \mathrm{~K}$. [e] B3LYP-D3BJ and MP2 with 6-311++G(d,p) basis set. 
Table S13. MP2/6-311++G(d,p) values for the equilibrium, zero-point energies, and Gibbs energies (346 K, 1 atm) for cyclooctanone BC1, its observed complexes with water and the corresponding gem-diols and transition states.

\begin{tabular}{|c|c|c|c|c|}
\hline & $E(\mathrm{~h})$ & $E_{0}(\mathrm{~h})$ & $G(\mathrm{~h})$ & $H(\mathrm{~h})$ \\
\hline \multicolumn{5}{|l|}{$1 w$} \\
\hline $\mathrm{H}_{2} \mathrm{O}$ & -76.274920 & -76.253239 & -76.275123 & -76.248847 \\
\hline CYO BC1 & -387.4441609 & -387.233322 & -387.273411 & -387.220772 \\
\hline $\mathrm{CYO}+\mathrm{H}_{2} \mathrm{O}$ & -463.7190813 & -463.486561 & -463.548534 & -463.469619 \\
\hline $1 w-1$ & -463.7312029 & -463.49571 & -463.542091 & -463.479019 \\
\hline $1 w-I I$ & -463.7309646 & -463.495588 & -463.542646 & -463.478822 \\
\hline TS & -463.653018 & -463.420164 & -463.462067 & -463.405802 \\
\hline Gemdiol I & -463.727441 & -463.488017 & -463.529646 & -463.473593 \\
\hline \multicolumn{5}{|l|}{$2 w$} \\
\hline $\mathrm{CYO}+\mathrm{H}_{2} \mathrm{O}+\mathrm{H}_{2} \mathrm{O}$ & -539.9940017 & -539.739800 & -539.823657 & -539.718466 \\
\hline $2 w-1$ & -540.0218546 & -539.760881 & -539.813030 & -539.740503 \\
\hline $2 w-I V$ & -540.0209348 & -539.760184 & -539.813003 & -539.739687 \\
\hline $2 w-I-T S$ & -539.9675021 & -539.709354 & -539.753531 & -539.693077 \\
\hline $2 w-I V-T S$ & -539.9630706 & -539.705207 & -539.749670 & -539.688764 \\
\hline Gemdiol1w-I & -540.0167509 & -539.751769 & -539.798198 & -539.733708 \\
\hline Gemdiol1w-III & -540.0166772 & -539.751700 & -539.798079 & -539.733665 \\
\hline
\end{tabular}


Figure S1. Top view of some cyclooctanone- $\mathrm{H}_{2} \mathrm{O}$ structures predicted differently from B3LYP-D3BJ and MP2 calculations.

$1 w-I$

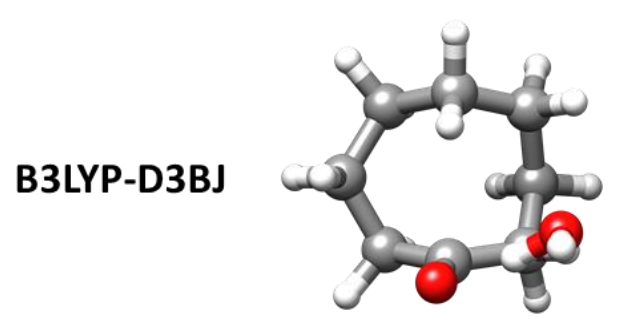

MP2

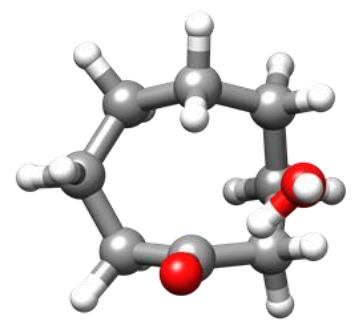

1w-IV
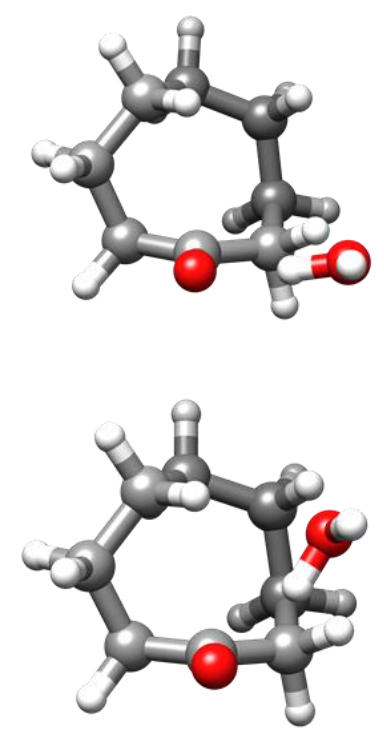

$1 w-V$
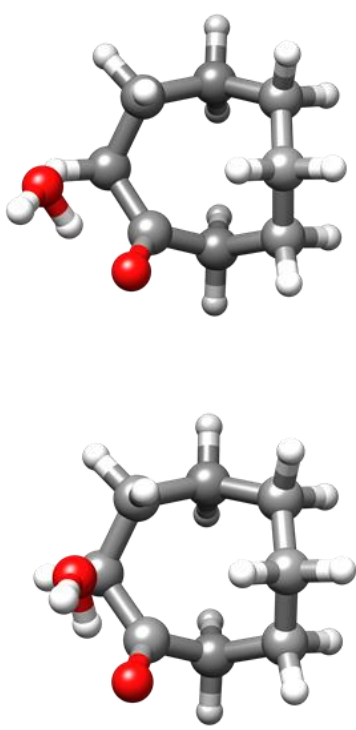
Figure S2. Overlays of the MP2/6-311++G(d,p) structures of the observed isomers of cyclooctanone- $\left(\mathrm{H}_{2} \mathrm{O}\right)_{1,2}$ with the $r_{s}$ positions of the atoms of water from experimental observations (blue spheres).
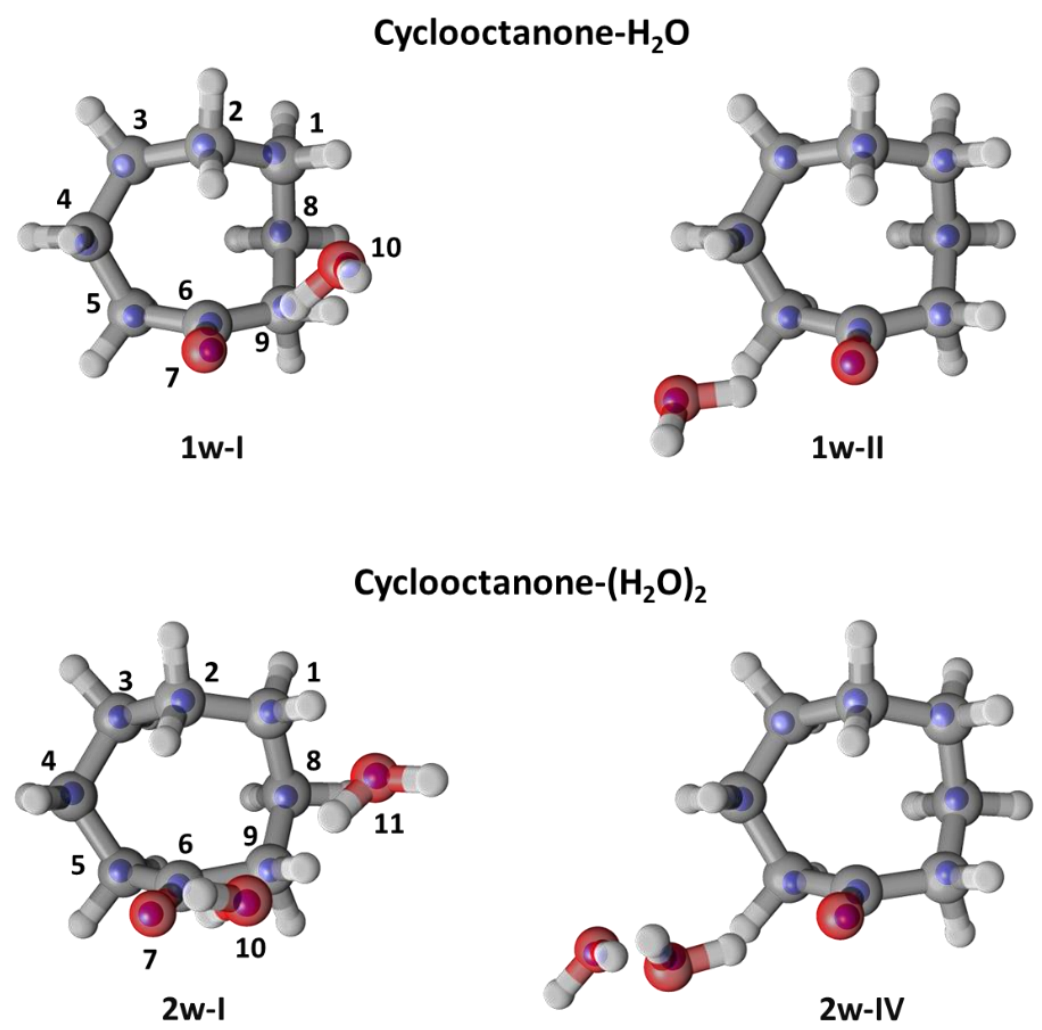
Figure S3. Plots of the reduced density gradient (RDG) versus $\operatorname{sign}(\lambda 2) \rho$ for the observed complexes of cyclooctanone- $\mathrm{H}_{2} \mathrm{O}$.

$1 w-I$

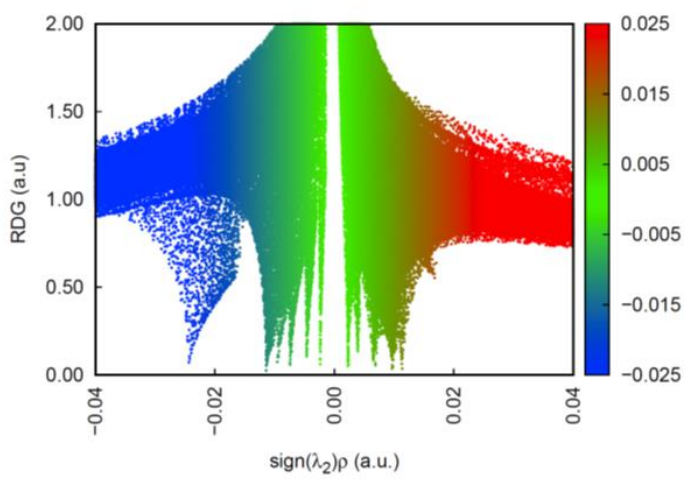

2w-I

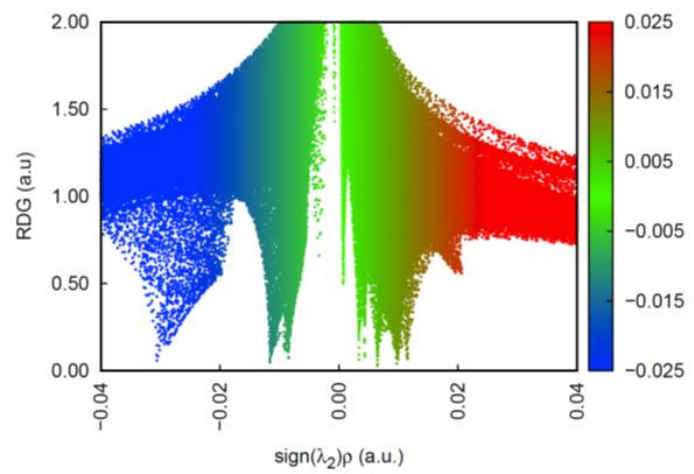

1w-II

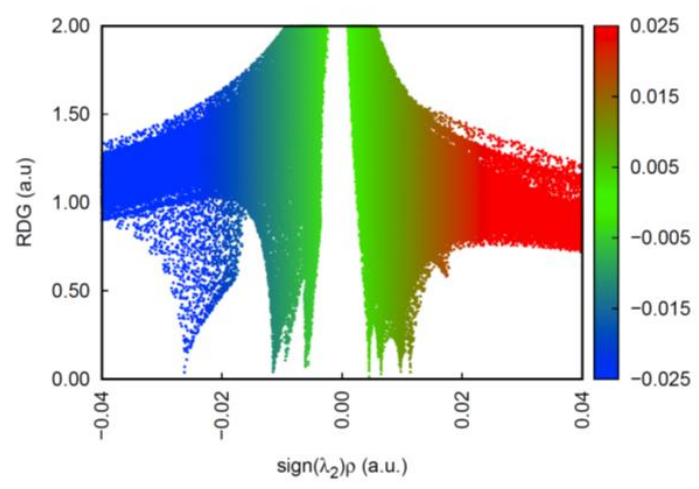

2w-IV

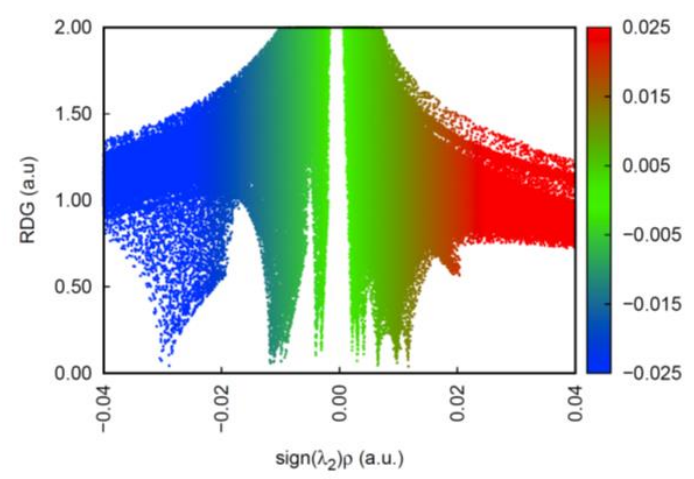


Table S14. Measured frequencies and residuals (in $\mathrm{MHz}$ ) for the rotational transitions of the parent species of $1 \mathrm{w}-\mathrm{I}$ complex.

\begin{tabular}{rccccccr}
\hline \hline$J^{\prime}$ & $\mathrm{K}_{-1}^{\prime}$ & $\mathrm{K}^{\prime}+1$ & $\mathrm{~J}^{\prime \prime}$ & $\mathrm{K}^{\prime \prime}-1$ & $\mathrm{~K}^{\prime \prime}+1$ & $\mathrm{~V}_{\text {obs }}$ & $\mathrm{V}_{\text {obs }} \mathrm{V}_{\text {calc }}$ \\
\hline 5 & 2 & 4 & 5 & 0 & 5 & 2508.8242 & 0.0006 \\
5 & 3 & 3 & 5 & 1 & 4 & 2748.5275 & 0.0037 \\
6 & 1 & 5 & 6 & 1 & 6 & 2760.4541 & 0.0005 \\
4 & 3 & 2 & 4 & 1 & 3 & 2798.8433 & 0.0055 \\
6 & 3 & 4 & 6 & 1 & 5 & 2837.4451 & -0.0094 \\
6 & 2 & 5 & 6 & 0 & 6 & 2966.5683 & -0.0001 \\
7 & 1 & 6 & 7 & 1 & 7 & 3375.6429 & -0.0038 \\
7 & 2 & 6 & 7 & 0 & 7 & 3472.0450 & -0.0011 \\
2 & 1 & 2 & 1 & 1 & 1 & 3644.8862 & -0.0027 \\
2 & 0 & 2 & 1 & 0 & 1 & 3756.7315 & -0.0016 \\
2 & 1 & 1 & 1 & 1 & 0 & 3950.5023 & 0.0017 \\
8 & 1 & 7 & 8 & 1 & 8 & 3962.1635 & -0.0121 \\
8 & 2 & 7 & 8 & 0 & 8 & 4003.5489 & 0.0191 \\
2 & 1 & 1 & 1 & 0 & 1 & 4444.3227 & 0.0076 \\
9 & 2 & 8 & 9 & 0 & 9 & 4547.5808 & -0.0014 \\
3 & 1 & 2 & 2 & 2 & 0 & 4831.6352 & 0.0041 \\
2 & 2 & 0 & 1 & 1 & 0 & 5014.4665 & -0.0037 \\
2 & 2 & 1 & 1 & 1 & 1 & 5126.3257 & -0.0012 \\
3 & 1 & 3 & 2 & 1 & 2 & 5444.3493 & -0.0001 \\
4 & 1 & 4 & 3 & 2 & 2 & 5491.1267 & -0.0003 \\
3 & 0 & 3 & 2 & 0 & 2 & 5548.9205 & -0.0020 \\
3 & 2 & 2 & 2 & 2 & 1 & 5696.5233 & -0.0028 \\
3 & 2 & 1 & 2 & 2 & 0 & 5844.0611 & 0.0011 \\
3 & 1 & 2 & 2 & 1 & 1 & 5895.6005 & -0.0002 \\
3 & 1 & 2 & 2 & 0 & 2 & 6583.1833 & 0.0006 \\
4 & 1 & 3 & 3 & 2 & 1 & 6782.7849 & 0.0042 \\
3 & 2 & 1 & 2 & 1 & 1 & 6908.0281 & -0.0015 \\
3 & 2 & 2 & 2 & 1 & 2 & 7177.9650 & 0.0009 \\
4 & 1 & 4 & 3 & 1 & 3 & 7224.7420 & 0.0003 \\
4 & 0 & 4 & 3 & 0 & 3 & 7292.2725 & -0.0007 \\
4 & 2 & 3 & 3 & 2 & 2 & 7562.8209 & 0.0017 \\
4 & 3 & 2 & 3 & 3 & 1 & 7660.1813 & 0.0001 \\
4 & 3 & 1 & 3 & 3 & 0 & 7700.9189 & -0.0039 \\
4 & 1 & 3 & 3 & 1 & 2 & 7795.2088 & -0.0009 \\
4 & 2 & 2 & 3 & 2 & 1 & 7864.6612 & -0.0005 \\
\hline \hline
\end{tabular}

Table S15. Measured frequencies and residuals (in $\mathrm{MHz}$ ) for the rotational transitions of the ${ }^{13} \mathrm{C}_{1}$ isotopologue of $1 \mathrm{w}-\mathrm{I}$.

\begin{tabular}{rcccccrr}
\hline \hline $\mathrm{J}^{\prime}$ & $\mathrm{K}_{-1}^{\prime}$ & $\mathrm{K}^{\prime}{ }_{+1}$ & $\mathrm{~J}^{\prime \prime}$ & $\mathrm{K}_{-1}$ & $\mathrm{~K}^{\prime \prime}{ }_{+1}$ & $\mathrm{~V}_{\mathrm{obs}}$ & $\mathrm{V}_{\text {obs }} \mathrm{V}_{\text {calc }}$ \\
\hline 2 & 0 & 2 & 1 & 0 & 1 & 3738.7530 & -0.0068 \\
2 & 1 & 1 & 1 & 1 & 0 & 3943.0333 & 0.0011 \\
3 & 1 & 3 & 2 & 1 & 2 & 5412.6096 & -0.0065 \\
3 & 0 & 3 & 2 & 0 & 2 & 5514.6313 & -0.0075 \\
3 & 2 & 2 & 2 & 2 & 1 & 5676.2325 & 0.0046 \\
3 & 1 & 2 & 2 & 1 & 1 & 5880.6994 & -0.0076 \\
4 & 1 & 4 & 3 & 1 & 3 & 7179.5199 & -0.0035 \\
4 & 0 & 4 & 3 & 0 & 3 & 7241.9788 & 0.0074 \\
4 & 2 & 3 & 3 & 2 & 2 & 7532.1636 & 0.0127 \\
4 & 2 & 2 & 3 & 2 & 1 & 7856.9471 & -0.0011 \\
\hline \hline
\end{tabular}


Table S16. Measured frequencies and residuals (in $\mathrm{MHz}$ ) for the rotational transitions of the ${ }^{13} \mathrm{C}_{2}$ isotopologue of $1 \mathrm{w}-\mathrm{l}$.

\begin{tabular}{|c|c|c|c|c|c|c|c|}
\hline$\overline{J^{\prime}}$ & $\mathrm{K}_{-1}^{\prime}$ & $\mathrm{K}^{\prime}+1$ & $\mathrm{~J}^{\prime \prime}$ & $\mathrm{K}^{\prime \prime}{ }_{-1}$ & $\mathrm{~K}^{\prime \prime}+1$ & $\mathrm{~V}_{\text {obs }}$ & $\mathrm{V}_{\text {obs }}-\mathrm{V}_{\text {calc }}$ \\
\hline 2 & 1 & 2 & 1 & 1 & 1 & 3632.4044 & 0.0113 \\
\hline 2 & 0 & 2 & 1 & 0 & 1 & 3743.3853 & -0.0054 \\
\hline 2 & 1 & 1 & 1 & 1 & 0 & 3936.9390 & 0.0045 \\
\hline 3 & 1 & 3 & 2 & 1 & 2 & 5425.4623 & 0.0083 \\
\hline 3 & 0 & 3 & 2 & 0 & 2 & 5528.5542 & -0.0057 \\
\hline 3 & 1 & 2 & 2 & 1 & 1 & 5874.9638 & 0.0058 \\
\hline 4 & 1 & 4 & 3 & 1 & 3 & 7199.4055 & -0.0072 \\
\hline 4 & 0 & 4 & 3 & 0 & 3 & 7265.3971 & -0.0006 \\
\hline 4 & 2 & 3 & 3 & 2 & 2 & 7536.5007 & 0.0030 \\
\hline 4 & 2 & 2 & 3 & 2 & 1 & 7839.1139 & -0.0068 \\
\hline
\end{tabular}

Table S17. Measured frequencies and residuals (in $\mathrm{MHz}$ ) for the rotational transitions of the ${ }^{13} \mathrm{C}_{3}$ isotopologue of $1 \mathrm{w}-\mathrm{I}$.

\begin{tabular}{|c|c|c|c|c|c|c|c|}
\hline $\mathrm{J}^{\prime}$ & $\mathrm{K}_{-1}^{\prime}$ & $\mathrm{K}^{\prime}+1$ & $\mathrm{~J}^{\prime \prime}$ & $\mathrm{K}_{-1}^{\prime \prime}$ & $\mathrm{K}^{\prime \prime}+1$ & V obs & $\mathrm{V}_{\text {obs }}-\mathrm{V}_{\text {calc }}$ \\
\hline 2 & 1 & 2 & 1 & 1 & 1 & 3615.4573 & -0.0044 \\
\hline 2 & 0 & 2 & 1 & 0 & 1 & 3725.8304 & -0.0032 \\
\hline 2 & 1 & 1 & 1 & 1 & 0 & 3913.1860 & 0.0027 \\
\hline 3 & 1 & 3 & 2 & 1 & 2 & 5401.5376 & 0.0136 \\
\hline 3 & 0 & 3 & 2 & 0 & 2 & 5506.8763 & 0.0032 \\
\hline 3 & 2 & 2 & 2 & 2 & 1 & 5646.4707 & 0.0030 \\
\hline 3 & 1 & 2 & 2 & 1 & 1 & 5841.6301 & -0.0009 \\
\hline 4 & 1 & 4 & 3 & 1 & 3 & 7169.3228 & -0.0129 \\
\hline 4 & 0 & 4 & 3 & 0 & 3 & 7239.3597 & 0.0044 \\
\hline 4 & 1 & 3 & 3 & 1 & 2 & 7727.7971 & -0.0040 \\
\hline 4 & 2 & 2 & 3 & 2 & 1 & 7786.2218 & 0.0008 \\
\hline
\end{tabular}

Table S18. Measured frequencies and residuals (in $\mathrm{MHz}$ ) for the rotational transitions of the ${ }^{13} \mathrm{C}_{4}$ isotopologue of $1 \mathrm{w}-\mathrm{l}$.

\begin{tabular}{ccccccrr}
\hline \hline $\mathrm{J}^{\prime}$ & $\mathrm{K}_{-1}^{\prime}$ & $\mathrm{K}^{\prime}{ }_{+1}$ & $\mathrm{~J}^{\prime \prime}$ & $\mathrm{K}^{\prime \prime} \mathrm{K}_{-1}$ & $\mathrm{~K}_{+1}$ & $\mathrm{~V}_{\mathrm{obs}}$ \\
\hline 2 & 0 & 2 & 1 & 0 & 1 & 3728.2151 & -0.0069 \\
3 & 1 & 3 & 2 & 1 & 2 & 5402.4724 & -0.0017 \\
3 & 0 & 3 & 2 & 0 & 2 & 5507.0763 & -0.0079 \\
3 & 2 & 2 & 2 & 2 & 1 & 5652.9768 & 0.0102 \\
3 & 2 & 1 & 2 & 2 & 0 & 5798.7743 & -0.0050 \\
3 & 1 & 2 & 2 & 1 & 1 & 5851.0732 & 0.0072 \\
4 & 0 & 4 & 3 & 0 & 3 & 7237.2112 & 0.0056 \\
4 & 1 & 3 & 3 & 1 & 2 & 7736.7913 \\
\hline \hline
\end{tabular}


Table S19. Measured frequencies and residuals (in $\mathrm{MHz}$ ) for the rotational transitions of the ${ }^{13} \mathrm{C}_{5}$ isotopologue of $1 \mathrm{w}-\mathrm{l}$.

\begin{tabular}{|c|c|c|c|c|c|c|c|}
\hline$\overline{\mathrm{J}^{\prime}}$ & $\mathrm{K}_{-1}^{\prime}$ & $\overline{\mathrm{K}^{\prime}+1}$ & $\mathrm{~J}^{\prime \prime}$ & $\mathrm{K}^{\prime \prime}{ }_{-1}$ & $\mathrm{~K}^{\prime \prime}+1$ & $\mathrm{~V}_{\mathrm{obs}}$ & $\mathrm{V}_{\mathrm{obs}}-\mathrm{V}_{\mathrm{calc}}$ \\
\hline 3 & 1 & 3 & 2 & 1 & 2 & 5415.0465 & -0.0039 \\
\hline 3 & 0 & 3 & 2 & 0 & 2 & 5518.4033 & -0.0053 \\
\hline 3 & 1 & 2 & 2 & 1 & 1 & 5872.8652 & 0.0003 \\
\hline 4 & 1 & 4 & 3 & 1 & 3 & 7184.4912 & 0.0036 \\
\hline 4 & 0 & 4 & 3 & 0 & 3 & 7249.7211 & 0.0021 \\
\hline 4 & 1 & 3 & 3 & 1 & 2 & 7761.4703 & 0.0011 \\
\hline 4 & 2 & 2 & 3 & 2 & 1 & 7839.5312 & -0.0002 \\
\hline
\end{tabular}

Table S20. Measured frequencies and residuals (in $\mathrm{MHz}$ ) for the rotational transitions of the ${ }^{13} \mathrm{C}_{6}$ isotopologue of $1 \mathrm{w}-\mathrm{I}$.

\begin{tabular}{rcccccrr}
\hline \hline $\mathrm{J}^{\prime}$ & $\mathrm{K}_{-1}^{\prime}$ & $\mathrm{K}^{\prime}{ }_{+1}$ & $\mathrm{~J}^{\prime \prime}$ & $\mathrm{K}^{\prime \prime}{ }_{-1}$ & $\mathrm{~K}^{\prime \prime}+1$ & $\mathrm{~V}_{\text {obs }}$ & $\mathrm{V}_{\text {obs }}-\mathrm{V}_{\text {calc }}$ \\
\hline 2 & 1 & 2 & 1 & 1 & 1 & 3638.5446 & -0.0072 \\
2 & 0 & 2 & 1 & 0 & 1 & 3750.4567 & 0.0092 \\
2 & 1 & 1 & 1 & 0 & 1 & 4436.3445 & -0.0046 \\
3 & 1 & 3 & 2 & 1 & 2 & 5434.4102 & -0.0034 \\
3 & 0 & 3 & 2 & 0 & 2 & 5538.1657 & 0.0021 \\
3 & 2 & 2 & 2 & 2 & 1 & 5688.3215 & -0.0080 \\
3 & 2 & 1 & 2 & 2 & 0 & 5838.4215 & -0.0040 \\
3 & 1 & 2 & 2 & 1 & 1 & 5888.0326 & 0.0068 \\
4 & 1 & 4 & 3 & 1 & 3 & 7210.9665 & -0.0024 \\
4 & 2 & 3 & 3 & 2 & 2 & 7551.2301 & 0.0069 \\
4 & 1 & 3 & 3 & 1 & 2 & 7783.5407 & 0.0030 \\
4 & 2 & 2 & 3 & 2 & 1 & 7857.1565 & -0.0013 \\
\hline \hline
\end{tabular}

Table S21. Measured frequencies and residuals (in $\mathrm{MHz}$ ) for the rotational transitions of the ${ }^{18} \mathrm{O}_{7}$ isotopologue of $1 \mathrm{w}-\mathrm{I}$.

\begin{tabular}{rccccccr}
\hline \hline$J^{\prime}$ & $\mathrm{K}_{-1}^{\prime}$ & $\mathrm{K}^{\prime}+1$ & $\mathrm{~J}^{\prime \prime}$ & $\mathrm{K}^{\prime \prime}{ }_{-1}$ & $\mathrm{~K}^{\prime \prime}+1$ & $\mathrm{~V}_{\text {obs }}$ & $\mathrm{V}_{\text {obs }}-\mathrm{V}_{\text {calc }}$ \\
\hline 2 & 1 & 2 & 1 & 1 & 1 & 3603.2484 & -0.0077 \\
2 & 0 & 2 & 1 & 0 & 1 & 3716.7426 & -0.0026 \\
2 & 1 & 1 & 1 & 1 & 0 & 3919.7221 & -0.0014 \\
2 & 2 & 0 & 1 & 1 & 0 & 4952.9241 & 0.0034 \\
2 & 2 & 1 & 1 & 1 & 1 & 5066.4216 & -0.0008 \\
3 & 1 & 3 & 2 & 1 & 2 & 5379.9010 & -0.0019 \\
3 & 0 & 3 & 2 & 0 & 2 & 5482.6281 & -0.0034 \\
3 & 2 & 2 & 2 & 2 & 1 & 5642.2137 & -0.0050 \\
3 & 2 & 1 & 2 & 2 & 0 & 5801.7358 & -0.0002 \\
3 & 1 & 2 & 2 & 1 & 1 & 5846.3069 & 0.0004 \\
3 & 1 & 2 & 2 & 0 & 2 & 6537.0054 & -0.0034 \\
4 & 1 & 4 & 3 & 1 & 3 & 7136.3186 & 0.0079 \\
4 & 0 & 4 & 3 & 0 & 3 & 7199.7662 & 0.0010 \\
4 & 2 & 3 & 3 & 2 & 2 & 7487.3392 & -0.0037 \\
4 & 3 & 2 & 3 & 3 & 1 & 7592.9400 & 0.0035 \\
4 & 3 & 1 & 3 & 3 & 0 & 7639.8016 & 0.0063 \\
4 & 1 & 3 & 3 & 1 & 2 & 7722.3707 & -0.0023 \\
4 & 2 & 2 & 3 & 2 & 1 & 7809.0513 & 0.0019 \\
\hline \hline
\end{tabular}

Table S22. Measured frequencies and residuals (in $\mathrm{MHz}$ ) for the rotational transitions of the ${ }^{13} \mathrm{C}_{8}$ isotopologue of $1 \mathrm{w}-\mathrm{I}$.

\begin{tabular}{|c|c|c|c|c|c|c|c|}
\hline $\mathrm{J}^{\prime}$ & $\mathrm{K}_{-1}^{\prime}$ & $\mathrm{K}_{+1}^{\prime}$ & $\mathrm{J \prime \prime}$ & $K^{\prime \prime}{ }_{-1}$ & $\mathrm{~K}^{\prime \prime}{ }_{+1}$ & $\mathrm{~V}_{\text {obs }}$ & $\mathrm{V}_{\text {obs }}-\mathrm{V}_{\text {calc }}$ \\
\hline 2 & 0 & 2 & 1 & 0 & 1 & 3742.1972 & -0.0053 \\
\hline 2 & 1 & 1 & 1 & 1 & 0 & 3933.8785 & 0.0072 \\
\hline 3 & 0 & 3 & 2 & 0 & 2 & 5527.6253 & -0.0010 \\
\hline 3 & 2 & 1 & 2 & 2 & 0 & 5821.4747 & 0.0023 \\
\hline 4 & 1 & 4 & 3 & 1 & 3 & 7199.8128 & 0.0200 \\
\hline 4 & 0 & 4 & 3 & 0 & 3 & 7265.1804 & 0.0055 \\
\hline 4 & 2 & 3 & 3 & 2 & 2 & 7533.6380 & 0.0102 \\
\hline 4 & 1 & 3 & 3 & 1 & 2 & 7761.9165 & -0.0088 \\
\hline
\end{tabular}


Table S23. Measured frequencies and residuals (in $\mathrm{MHz}$ ) for the rotational transitions of the ${ }^{13} \mathrm{C}_{9}$ isotopologue of $1 \mathrm{w}-\mathrm{I}$.

\begin{tabular}{rcccccrr}
\hline \hline $\mathrm{J}^{\prime}$ & $\mathrm{K}_{-1}^{\prime}$ & $\mathrm{K}_{+1}^{\prime}$ & $\mathrm{J}^{\prime \prime}$ & $\mathrm{K}_{-1}^{\prime}$ & $\mathrm{K}_{+1}$ & $\mathrm{~V}_{\text {obs }}$ & $\mathrm{V}_{\text {obs }}-\mathrm{V}_{\text {calc }}$ \\
\hline 2 & 0 & 2 & 1 & 0 & 1 & 3746.2090 & -0.0035 \\
3 & 1 & 3 & 2 & 1 & 2 & 5433.7298 & -0.0013 \\
3 & 0 & 3 & 2 & 0 & 2 & 5536.8839 & -0.0072 \\
3 & 2 & 2 & 2 & 2 & 1 & 5677.7345 & 0.0092 \\
3 & 2 & 1 & 2 & 2 & 0 & 5818.4862 & -0.0037 \\
3 & 1 & 2 & 2 & 1 & 1 & 5871.2881 & -0.0007 \\
4 & 1 & 4 & 3 & 1 & 3 & 7212.0717 & 0.0033 \\
4 & 2 & 3 & 3 & 2 & 2 & 7539.3652 & -0.0008 \\
4 & 1 & 3 & 3 & 1 & 2 & 7766.3352 & 0.0028 \\
4 & 2 & 2 & 3 & 2 & 1 & 7828.7920 & -0.0008 \\
\hline \hline
\end{tabular}

Table S24. Measured frequencies and residuals (in $\mathrm{MHz}$ ) for the rotational transitions of the ${ }^{18} \mathrm{O}_{10}$ isotopologue of $1 \mathrm{w}-\mathrm{I}$.

\begin{tabular}{rcccccrr}
\hline \hline $\mathrm{J}^{\prime}$ & $\mathrm{K}_{-1}^{\prime}$ & $\mathrm{K}^{\prime}{ }_{+1}$ & $\mathrm{~J}^{\prime \prime}$ & $\mathrm{K}^{\prime \prime}{ }_{-1}$ & $\mathrm{~K}^{\prime \prime}{ }_{+1}$ & $\mathrm{~V}_{\mathrm{obs}}$ & $\mathrm{V}_{\text {obs }}-\mathrm{V}_{\text {calc }}$ \\
\hline 2 & 1 & 2 & 1 & 1 & 1 & 3521.3346 & -0.0021 \\
2 & 0 & 2 & 1 & 0 & 1 & 3628.4871 & -0.0026 \\
2 & 1 & 1 & 1 & 1 & 0 & 3800.2624 & 0.0009 \\
2 & 1 & 1 & 1 & 0 & 1 & 4313.5677 & -0.0028 \\
2 & 2 & 0 & 1 & 1 & 0 & 4954.0964 & 0.0039 \\
2 & 2 & 1 & 1 & 1 & 1 & 5061.2555 & -0.0026 \\
3 & 1 & 3 & 2 & 1 & 2 & 5263.6366 & -0.0010 \\
3 & 0 & 3 & 2 & 0 & 2 & 5371.9241 & 0.0002 \\
3 & 2 & 2 & 2 & 2 & 1 & 5491.1833 & 0.0007 \\
3 & 2 & 1 & 2 & 2 & 0 & 5610.3726 & 0.0010 \\
3 & 1 & 2 & 2 & 1 & 1 & 5677.1935 & 0.0014 \\
3 & 1 & 2 & 2 & 0 & 2 & 6362.2744 & 0.0015 \\
4 & 1 & 3 & 3 & 2 & 1 & 6432.7594 & 0.0011 \\
3 & 2 & 1 & 2 & 1 & 1 & 6764.2020 & -0.0006 \\
4 & 1 & 4 & 3 & 1 & 3 & 6989.8041 & -0.0017 \\
4 & 0 & 4 & 3 & 0 & 3 & 7067.8938 & 0.0034 \\
4 & 2 & 3 & 3 & 2 & 2 & 7295.9891 & -0.0042 \\
4 & 3 & 2 & 3 & 3 & 1 & 7374.2281 & 0.0067 \\
4 & 3 & 1 & 3 & 3 & 0 & 7402.0880 & -0.0061 \\
4 & 1 & 3 & 3 & 1 & 2 & 7519.7712 & 0.0024 \\
4 & 2 & 2 & 3 & 2 & 1 & 7548.8622 & -0.0014 \\
\hline \hline
\end{tabular}


Table S25. Measured frequencies and residuals (in $\mathrm{MHz}$ ) for the rotational transitions of the parent species of $1 \mathrm{w}-\mathrm{Il}$ complex.

\begin{tabular}{|c|c|c|c|c|c|c|c|}
\hline$\overline{\mathrm{J}^{\prime}}$ & $\mathrm{K}_{-1}^{\prime}$ & $\mathrm{K}_{+1}^{\prime}$ & J" & $\mathrm{K}^{\prime \prime}{ }_{-1}$ & $\mathrm{~K}^{\prime \prime}{ }_{+1}$ & V & $\mathrm{V}_{\text {obs }}-\mathrm{V}_{\text {calc }}$ \\
\hline 4 & 2 & 3 & 4 & 1 & 3 & 2184.8452 & -0.0093 \\
\hline 5 & 1 & 4 & 5 & 1 & 5 & 2427.7271 & 0.0128 \\
\hline 5 & 2 & 3 & 5 & 1 & 4 & 2462.0655 & 0.0073 \\
\hline 4 & 2 & 2 & 4 & 1 & 3 & 2473.5575 & -0.0028 \\
\hline 3 & 2 & 2 & 3 & 1 & 2 & 2484.2314 & -0.0092 \\
\hline 1 & 1 & 1 & 0 & 0 & 0 & 2487.2887 & 0.0067 \\
\hline 3 & 2 & 1 & 3 & 1 & 2 & 2584.7082 & 0.0042 \\
\hline 6 & 2 & 4 & 6 & 1 & 5 & 2596.1925 & 0.0070 \\
\hline 1 & 1 & 0 & 0 & 0 & 0 & 2652.0504 & -0.0044 \\
\hline 7 & 2 & 5 & 7 & 1 & 6 & 2909.4702 & 0.0031 \\
\hline 2 & 1 & 2 & 1 & 1 & 1 & 2997.9850 & -0.0026 \\
\hline 2 & 0 & 2 & 1 & 0 & 1 & 3142.2593 & -0.0033 \\
\hline 8 & 3 & 6 & 8 & 2 & 6 & 3177.6994 & 0.0146 \\
\hline 2 & 1 & 1 & 1 & 1 & 0 & 3327.5334 & 0.0008 \\
\hline 6 & 1 & 5 & 6 & 1 & 6 & 3332.6745 & -0.0168 \\
\hline 3 & 2 & 2 & 3 & 1 & 3 & 3471.2657 & -0.0006 \\
\hline 7 & 3 & 5 & 7 & 2 & 5 & 3691.3219 & 0.0028 \\
\hline 4 & 2 & 3 & 4 & 1 & 4 & 3821.4252 & 0.0103 \\
\hline 3 & 0 & 3 & 2 & 1 & 2 & 3902.5391 & 0.0044 \\
\hline 2 & 1 & 2 & 1 & 0 & 1 & 3903.8872 & 0.0003 \\
\hline 2 & 2 & 1 & 2 & 0 & 2 & 3973.6299 & 0.0002 \\
\hline 7 & 3 & 4 & 7 & 2 & 5 & 3993.6035 & 0.0065 \\
\hline 4 & 1 & 3 & 3 & 2 & 1 & 4024.1483 & -0.0107 \\
\hline 3 & 2 & 2 & 3 & 0 & 3 & 4053.5996 & 0.0013 \\
\hline 4 & 1 & 3 & 3 & 2 & 2 & 4124.6137 & -0.0087 \\
\hline 6 & 3 & 4 & 6 & 2 & 4 & 4133.0798 & -0.0103 \\
\hline 4 & 2 & 3 & 4 & 0 & 4 & 4225.9460 & -0.0021 \\
\hline 6 & 3 & 3 & 6 & 2 & 4 & 4260.5214 & -0.0046 \\
\hline 7 & 1 & 6 & 7 & 1 & 7 & 4310.8999 & 0.0012 \\
\hline 2 & 1 & 1 & 1 & 0 & 1 & 4398.2061 & 0.0014 \\
\hline 5 & 3 & 3 & 5 & 2 & 3 & 4479.3536 & 0.0025 \\
\hline 3 & 1 & 3 & 2 & 1 & 2 & 4484.8649 & -0.0018 \\
\hline 5 & 2 & 4 & 5 & 0 & 5 & 4519.9818 & -0.0066 \\
\hline 5 & 3 & 2 & 5 & 2 & 3 & 4523.2215 & 0.0162 \\
\hline 3 & 0 & 3 & 2 & 0 & 2 & 4664.1583 & -0.0008 \\
\hline 4 & 3 & 2 & 4 & 2 & 2 & 4717.3730 & -0.0035 \\
\hline 3 & 2 & 2 & 2 & 2 & 1 & 4744.1217 & -0.0059 \\
\hline 3 & 2 & 1 & 2 & 2 & 0 & 4824.0920 & -0.0019 \\
\hline 3 & 3 & 1 & 3 & 2 & 1 & 4853.0062 & -0.0056 \\
\hline 6 & 2 & 5 & 6 & 0 & 6 & 4943.4089 & -0.0230 \\
\hline 3 & 1 & 2 & 2 & 1 & 1 & 4977.5748 & 0.0002 \\
\hline 3 & 1 & 3 & 2 & 0 & 2 & 5246.4874 & -0.0036 \\
\hline 8 & 1 & 7 & 8 & 1 & 8 & 5315.9815 & 0.0129 \\
\hline 4 & 0 & 4 & 3 & 1 & 3 & 5554.7974 & 0.0023 \\
\hline 5 & 1 & 4 & 4 & 2 & 2 & 5737.9504 & -0.0008 \\
\hline 4 & 1 & 4 & 3 & 1 & 3 & 5959.3283 & 0.0000 \\
\hline 5 & 1 & 4 & 4 & 2 & 3 & 6026.6635 & 0.0066 \\
\hline 2 & 2 & 1 & 1 & 1 & 0 & 6045.2288 & 0.0086 \\
\hline 2 & 2 & 0 & 1 & 1 & 0 & 6065.7179 & 0.0006 \\
\hline 4 & 0 & 4 & 3 & 0 & 3 & 6137.1274 & 0.0003 \\
\hline 2 & 2 & 1 & 1 & 1 & 1 & 6209.9956 & 0.0027 \\
\hline 2 & 2 & 0 & 1 & 1 & 1 & 6230.4958 & 0.0058 \\
\hline 3 & 1 & 2 & 2 & 0 & 2 & 6233.5216 & 0.0049 \\
\hline 8 & 4 & 5 & 8 & 3 & 5 & 6308.0205 & -0.0060 \\
\hline 4 & 2 & 3 & 3 & 2 & 2 & 6309.4754 & -0.0016 \\
\hline 4 & 3 & 2 & 3 & 3 & 1 & 6362.0840 & 0.0000 \\
\hline 4 & 3 & 1 & 3 & 3 & 0 & 6371.6343 & -0.0013 \\
\hline 4 & 2 & 2 & 3 & 2 & 1 & 6497.7200 & 0.0007 \\
\hline 4 & 1 & 4 & 3 & 0 & 3 & 6541.6560 & -0.0043 \\
\hline 8 & 3 & 6 & 8 & 1 & 7 & 6600.3248 & 0.0094 \\
\hline 7 & 3 & 5 & 7 & 1 & 6 & 6600.7686 & -0.0177 \\
\hline 4 & 1 & 3 & 3 & 1 & 2 & 6608.8626 & -0.0005 \\
\hline
\end{tabular}




\begin{tabular}{rrrrrrrr}
6 & 3 & 4 & 6 & 1 & 5 & 6729.2872 & 0.0116 \\
6 & 4 & 3 & 6 & 3 & 3 & 6743.0804 & -0.0030 \\
5 & 4 & 2 & 5 & 3 & 2 & 6831.6052 & 0.0078 \\
4 & 4 & 1 & 4 & 3 & 1 & 6873.9805 & -0.0015 \\
5 & 3 & 3 & 5 & 1 & 4 & 6941.3960 & -0.0133 \\
5 & 0 & 5 & 4 & 1 & 4 & 7162.7628 & 0.0112 \\
4 & 3 & 2 & 4 & 1 & 3 & 7190.9347 & -0.0021 \\
6 & 1 & 5 & 5 & 2 & 3 & 7311.6552 & -0.0062 \\
5 & 1 & 5 & 4 & 1 & 4 & 7420.3642 & 0.0067 \\
3 & 2 & 2 & 2 & 1 & 1 & 7461.8113 & -0.0039 \\
3 & 2 & 1 & 2 & 1 & 1 & 7562.2768 & -0.0018 \\
5 & 0 & 5 & 4 & 0 & 4 & 7567.2902 & 0.0053 \\
5 & 1 & 5 & 4 & 0 & 4 & 7824.8924 & 0.0016 \\
5 & 2 & 4 & 4 & 2 & 3 & 7861.3225 & -0.0026 \\
5 & 4 & 2 & 4 & 4 & 1 & 7952.2990 & 0.0055 \\
5 & 4 & 1 & 4 & 4 & 0 & 7953.1185 & -0.0058 \\
3 & 2 & 2 & 2 & 1 & 2 & 7956.1390 & 0.0060 \\
5 & 3 & 3 & 4 & 3 & 2 & 7961.9820 & -0.0020 \\
5 & 3 & 2 & 4 & 3 & 1 & 7994.6755 & -0.0026 \\
\hline \hline
\end{tabular}

Table S26. Measured frequencies and residuals (in $\mathrm{MHz}$ ) for the rotational transitions of the ${ }^{13} \mathrm{C}_{1}$ isotopologue of $1 \mathrm{w}-\mathrm{II}$.

\begin{tabular}{rcccccrr}
\hline \hline $\mathrm{J}^{\prime}$ & $\mathrm{K}_{-1}^{\prime}$ & $\mathrm{K}^{\prime}{ }_{+1}$ & $\mathrm{~J}^{\prime \prime}$ & $\mathrm{K}^{\prime \prime}{ }_{-1}$ & $\mathrm{~K}^{\prime \prime}{ }_{+1}$ & $\mathrm{~V}_{\text {obs }}$ & $\mathrm{V}_{\text {obs }} \mathrm{V}_{\text {calc }}$ \\
\hline 2 & 0 & 2 & 1 & 0 & 1 & 3111.1693 & -0.0106 \\
2 & 1 & 1 & 1 & 1 & 0 & 3291.6920 & 0.0036 \\
3 & 1 & 3 & 2 & 1 & 2 & 4442.8858 & -0.0032 \\
3 & 0 & 3 & 2 & 0 & 2 & 4619.8962 & -0.0070 \\
3 & 2 & 2 & 2 & 2 & 1 & 4695.9507 & -0.0097 \\
3 & 1 & 2 & 2 & 1 & 1 & 4924.5291 & 0.0056 \\
4 & 1 & 4 & 3 & 1 & 3 & 5904.2976 & -0.0030 \\
4 & 0 & 4 & 3 & 0 & 3 & 6081.5886 & -0.0003 \\
4 & 2 & 3 & 3 & 2 & 2 & 6246.0612 & 0.0007 \\
4 & 1 & 3 & 3 & 1 & 2 & 6539.6421 & 0.0029 \\
5 & 1 & 5 & 4 & 1 & 4 & 7352.8007 & 0.0112 \\
\hline \hline
\end{tabular}

Table S27. Measured frequencies and residuals (in $\mathrm{MHz}$ ) for the rotational transitions of the ${ }^{13} \mathrm{C}_{2}$ isotopologue of $1 \mathrm{w}-\mathrm{II}$.

\begin{tabular}{rccccccr}
\hline \hline $\mathrm{J}^{\prime}$ & $\mathrm{K}_{-1}^{\prime}$ & $\mathrm{K}^{\prime}{ }_{+1}$ & $\mathrm{~J}^{\prime \prime}$ & $\mathrm{K}_{-1}{ }_{-1}$ & $\mathrm{~K}_{+1} \mathrm{~V}_{\text {obs }}$ & $\mathrm{V}_{\text {obs }} \mathrm{V}_{\text {calc }}$ \\
\hline 2 & 1 & 2 & 1 & 1 & 1 & 2983.9740 & -0.0039 \\
3 & 1 & 3 & 2 & 1 & 2 & 4463.9409 & -0.0042 \\
3 & 0 & 3 & 2 & 0 & 2 & 4641.6264 & -0.0034 \\
3 & 2 & 1 & 2 & 2 & 0 & 4800.3326 & -0.0054 \\
3 & 1 & 2 & 2 & 1 & 1 & 4952.4018 & -0.0056 \\
4 & 1 & 4 & 3 & 1 & 3 & 5931.5874 & -0.0048 \\
4 & 0 & 4 & 3 & 0 & 3 & 6107.7367 & 0.0020 \\
4 & 2 & 3 & 3 & 2 & 2 & 6278.7393 & -0.0029 \\
4 & 2 & 2 & 3 & 2 & 1 & 6465.5346 & 0.0137 \\
4 & 1 & 3 & 3 & 1 & 2 & 6575.5105 & -0.0048 \\
5 & 1 & 5 & 4 & 1 & 4 & 7385.9144 & -0.0007 \\
5 & 0 & 5 & 4 & 0 & 4 & 7531.4140 & 0.0085 \\
5 & 2 & 4 & 4 & 2 & 3 & 7823.1038 & 0.0024 \\
\hline \hline
\end{tabular}


Table S28. Measured frequencies and residuals (in $\mathrm{MHz}$ ) for the rotational transitions of the ${ }^{13} \mathrm{C}_{3}$ isotopologue of $1 \mathrm{w}-\mathrm{II}$.

\begin{tabular}{rcccccrr}
\hline \hline $\mathrm{J}^{\prime}$ & $\mathrm{K}_{-1}^{\prime}$ & $\mathrm{K}^{\prime}{ }_{+1}$ & $\mathrm{~J}^{\prime \prime}$ & $\mathrm{K}_{-1}^{\prime \prime}$ & $\mathrm{K}^{\prime \prime}{ }_{1}$ & $\mathrm{~V}_{\text {obs }}$ & $\mathrm{V}_{\text {obs }} \mathrm{V}_{\text {calc }}$ \\
\hline 2 & 1 & 2 & 1 & 1 & 1 & 2983.9740 & 0.0078 \\
2 & 0 & 2 & 1 & 0 & 1 & 3130.1970 & -0.0090 \\
2 & 1 & 1 & 1 & 1 & 0 & 3319.9132 & 0.0056 \\
3 & 1 & 3 & 2 & 1 & 2 & 4463.1261 & -0.0069 \\
3 & 0 & 3 & 2 & 0 & 2 & 4643.2772 & -0.0019 \\
3 & 2 & 1 & 2 & 2 & 0 & 4812.5154 & 0.0110 \\
3 & 1 & 2 & 2 & 1 & 1 & 4965.2728 & 0.0037 \\
4 & 1 & 4 & 3 & 1 & 3 & 5929.2499 & -0.0023 \\
4 & 0 & 4 & 3 & 0 & 3 & 6105.3787 & -0.0072 \\
4 & 2 & 3 & 3 & 2 & 2 & 6286.8562 & -0.0064 \\
4 & 1 & 3 & 3 & 1 & 2 & 6590.5982 & -0.0030 \\
5 & 1 & 5 & 4 & 1 & 4 & 7381.3903 & 0.0022 \\
5 & 0 & 5 & 4 & 0 & 4 & 7524.2410 & 0.0120 \\
5 & 2 & 4 & 4 & 2 & 3 & 7831.5317 & -0.0043 \\
\hline \hline
\end{tabular}

Table S29. Measured frequencies and residuals (in $\mathrm{MHz}$ ) for the rotational transitions of the ${ }^{13} \mathrm{C}_{4}$ isotopologue of $1 \mathrm{w}-\mathrm{II}$.

\begin{tabular}{rccccccr}
\hline \hline $\mathrm{J}^{\prime}$ & $\mathrm{K}_{-1}^{\prime}$ & $\mathrm{K}^{\prime}{ }_{+1}$ & $\mathrm{~J}^{\prime \prime}$ & $\mathrm{K}^{\prime \prime}{ }_{-1}$ & $\mathrm{~K}^{\prime \prime}{ }_{+1}$ & $\mathrm{~V}_{\text {obs }}$ & $\mathrm{V}_{\text {obs }}-\mathrm{V}_{\text {calc }}$ \\
\hline 2 & 1 & 2 & 1 & 1 & 1 & 2989.3784 & -0.0047 \\
2 & 0 & 2 & 1 & 0 & 1 & 3134.5413 & -0.0025 \\
3 & 1 & 3 & 2 & 1 & 2 & 4471.5523 & -0.0066 \\
3 & 0 & 3 & 2 & 0 & 2 & 4650.9790 & -0.0048 \\
3 & 2 & 2 & 2 & 2 & 1 & 4733.6249 & 0.0158 \\
3 & 2 & 1 & 2 & 2 & 0 & 4816.2237 & -0.0084 \\
3 & 1 & 2 & 2 & 1 & 1 & 4968.9480 & 0.0021 \\
4 & 1 & 4 & 3 & 1 & 3 & 5940.9591 & -0.0004 \\
4 & 0 & 4 & 3 & 0 & 3 & 6117.3472 & -0.0037 \\
4 & 2 & 3 & 3 & 2 & 2 & 6294.8967 & -0.0011 \\
4 & 2 & 2 & 3 & 2 & 1 & 6488.8839 & 0.0013 \\
4 & 1 & 3 & 3 & 1 & 2 & 6596.2917 & 0.0004 \\
5 & 1 & 5 & 4 & 1 & 4 & 7396.6269 & 0.0075 \\
5 & 0 & 5 & 4 & 0 & 4 & 7540.7105 & 0.0009 \\
5 & 2 & 4 & 4 & 2 & 3 & 7842.2285 & -0.0017 \\
\hline \hline
\end{tabular}

Table S30. Measured frequencies and residuals (in $\mathrm{MHz}$ ) for the rotational transitions of the ${ }^{13} \mathrm{C}_{5}$ isotopologue of $1 \mathrm{w}-\mathrm{II}$.

\begin{tabular}{|c|c|c|c|c|c|c|c|}
\hline $\mathrm{J}^{\prime}$ & $\mathrm{K}_{-1}^{\prime}$ & $\mathrm{K}^{\prime}+1$ & $\mathrm{~J}^{\prime \prime}$ & $\mathrm{K}^{\prime \prime}$ & $\mathrm{K}^{\prime \prime}+1$ & $\mathrm{~V}_{\text {obs }}$ & $\mathrm{V}_{\text {obs }}-\mathrm{V}_{\text {calc }}$ \\
\hline 2 & 1 & 2 & 1 & 1 & 1 & 2993.6036 & -0.0082 \\
\hline 2 & 0 & 2 & 1 & 0 & 1 & 3135.9254 & -0.0054 \\
\hline 3 & 1 & 3 & 2 & 1 & 2 & 4478.5619 & -0.0009 \\
\hline 3 & 0 & 3 & 2 & 0 & 2 & 4655.7054 & -0.0024 \\
\hline 3 & 1 & 2 & 2 & 1 & 1 & 4964.1087 & 0.0011 \\
\hline 4 & 1 & 4 & 3 & 1 & 3 & 5951.3524 & 0.0053 \\
\hline 4 & 0 & 4 & 3 & 0 & 3 & 6127.4729 & 0.0012 \\
\hline 4 & 2 & 3 & 3 & 2 & 2 & 6296.2749 & 0.0057 \\
\hline 4 & 2 & 2 & 3 & 2 & 1 & 6480.6128 & -0.0001 \\
\hline 4 & 1 & 3 & 3 & 1 & 2 & 6591.5466 & 0.0011 \\
\hline 5 & 1 & 5 & 4 & 1 & 4 & 7410.9491 & 0.0011 \\
\hline 5 & 0 & 5 & 4 & 0 & 4 & 7556.9950 & -0.0005 \\
\hline 5 & 2 & 4 & 4 & 2 & 3 & 7845.3652 & -0.0045 \\
\hline
\end{tabular}


Table S31. Measured frequencies and residuals (in $\mathrm{MHz}$ ) for the rotational transitions of the ${ }^{13} \mathrm{C}_{6}$ isotopologue of $1 \mathrm{w}-\mathrm{II}$.

\begin{tabular}{|c|c|c|c|c|c|c|c|}
\hline $\mathrm{J}^{\prime}$ & $\mathrm{K}_{-1}^{\prime}$ & $\mathrm{K}^{\prime}+1$ & $\mathrm{~J}^{\prime \prime}$ & $\mathrm{K}_{-1}^{\prime \prime}$ & $\mathrm{K}_{+1}^{\prime \prime}$ & V & $\mathrm{V}_{\text {obs }}-\mathrm{V}_{\text {calc }}$ \\
\hline 2 & 0 & 2 & 1 & 0 & 1 & 3139.0782 & -0.0148 \\
\hline 3 & 1 & 3 & 2 & 1 & 2 & 4479.1862 & -0.0043 \\
\hline 3 & 0 & 3 & 2 & 0 & 2 & 4658.6856 & -0.0045 \\
\hline 3 & 2 & 2 & 2 & 2 & 1 & 4739.8553 & 0.0101 \\
\hline 3 & 1 & 2 & 2 & 1 & 1 & 4974.2869 & 0.0012 \\
\hline 4 & 1 & 4 & 3 & 1 & 3 & 5951.4755 & -0.0038 \\
\hline 4 & 0 & 4 & 3 & 0 & 3 & 6128.8350 & 0.0030 \\
\hline 4 & 2 & 3 & 3 & 2 & 2 & 6303.5261 & 0.0059 \\
\hline 4 & 2 & 2 & 3 & 2 & 1 & 6494.3431 & -0.0009 \\
\hline 4 & 1 & 3 & 3 & 1 & 2 & 6604.0051 & -0.0009 \\
\hline 5 & 1 & 5 & 4 & 1 & 4 & 7410.1991 & 0.0052 \\
\hline 5 & 2 & 4 & 4 & 2 & 3 & 7853.4877 & -0.0035 \\
\hline
\end{tabular}

Table S32. Measured frequencies and residuals (in $\mathrm{MHz}$ ) for the rotational transitions of the ${ }^{18} \mathrm{O}_{7}$ isotopologue of $1 \mathrm{w}-\mathrm{II}$.

\begin{tabular}{rccccccr}
\hline \hline $\mathrm{J}^{\prime}$ & $\mathrm{K}_{-1}^{\prime}$ & $\mathrm{K}_{+1}^{\prime}$ & $\mathrm{J}^{\prime \prime}$ & $\mathrm{K}_{-1}^{\prime \prime}$ & $\mathrm{K}_{+1}{ }_{+1}$ & $\mathrm{~V}_{\text {obs }}$ & $\mathrm{V}_{\text {obs }}-\mathrm{V}_{\text {calc }}$ \\
\hline 1 & 1 & 0 & 0 & 0 & 0 & 2621.5354 & 0.0008 \\
2 & 1 & 2 & 1 & 1 & 1 & 2972.9976 & -0.0045 \\
2 & 0 & 2 & 1 & 0 & 1 & 3117.0083 & 0.0021 \\
2 & 1 & 1 & 1 & 1 & 0 & 3302.8462 & 0.0074 \\
2 & 1 & 1 & 1 & 0 & 1 & 4355.4006 & -0.0100 \\
3 & 1 & 3 & 2 & 1 & 2 & 4447.1509 & -0.0040 \\
3 & 0 & 3 & 2 & 0 & 2 & 4625.3485 & -0.0027 \\
3 & 2 & 2 & 2 & 2 & 1 & 4706.8767 & 0.0085 \\
3 & 2 & 1 & 2 & 2 & 0 & 4788.3890 & 0.0060 \\
3 & 1 & 2 & 2 & 1 & 1 & 4940.2374 & 0.0022 \\
4 & 1 & 4 & 3 & 1 & 3 & 5908.6912 & -0.0014 \\
2 & 2 & 0 & 1 & 1 & 0 & 5986.7031 & 0.0016 \\
4 & 0 & 4 & 3 & 0 & 3 & 6084.1908 & -0.0044 \\
2 & 2 & 1 & 1 & 1 & 1 & 6130.7114 & 0.0051 \\
3 & 1 & 2 & 2 & 0 & 2 & 6178.6308 & -0.0087 \\
4 & 2 & 2 & 3 & 2 & 1 & 6450.9612 & 0.0023 \\
4 & 1 & 3 & 3 & 1 & 2 & 6558.4280 & 0.0002 \\
5 & 1 & 5 & 4 & 1 & 4 & 7356.6518 & 0.0064 \\
5 & 0 & 5 & 4 & 0 & 4 & 7500.3524 & -0.0038 \\
5 & 2 & 4 & 4 & 2 & 3 & 7798.3027 & -0.0016 \\
\hline \hline
\end{tabular}


Table S33. Measured frequencies and residuals (in $\mathrm{MHz}$ ) for the rotational transitions of the ${ }^{13} \mathrm{C}_{8}$ isotopologue of $1 \mathrm{w}-\mathrm{II}$.

\begin{tabular}{|c|c|c|c|c|c|c|c|}
\hline $\mathrm{J}^{\prime}$ & $\mathrm{K}_{-1}^{\prime}$ & $\mathrm{K}^{\prime}+1$ & $\mathrm{~J}^{\prime \prime}$ & $\mathrm{K}_{-1}^{\prime \prime}$ & $\mathrm{K}^{\prime \prime}+1$ & V & $\mathrm{V}_{\text {obs }}-\mathrm{V}_{\text {calc }}$ \\
\hline 2 & 0 & 2 & 1 & 0 & 1 & 3117.9707 & -0.0015 \\
\hline 2 & 1 & 1 & 1 & 1 & 0 & 3300.9318 & 0.0060 \\
\hline 3 & 1 & 3 & 2 & 1 & 2 & 4450.8222 & 0.0059 \\
\hline 3 & 0 & 3 & 2 & 0 & 2 & 4628.7642 & 0.0019 \\
\hline 3 & 2 & 1 & 2 & 2 & 0 & 4785.2599 & -0.0115 \\
\hline 3 & 1 & 2 & 2 & 1 & 1 & 4937.9805 & 0.0048 \\
\hline 4 & 1 & 4 & 3 & 1 & 3 & 5914.3425 & -0.0010 \\
\hline 4 & 0 & 4 & 3 & 0 & 3 & 6091.4710 & -0.0038 \\
\hline 4 & 2 & 3 & 3 & 2 & 2 & 6260.3460 & -0.0037 \\
\hline 4 & 2 & 2 & 3 & 2 & 1 & 6444.7791 & 0.0096 \\
\hline 4 & 1 & 3 & 3 & 1 & 2 & 6556.7191 & -0.0005 \\
\hline 5 & 1 & 5 & 4 & 1 & 4 & 7364.6701 & 0.0016 \\
\hline 5 & 0 & 5 & 4 & 0 & 4 & 7511.7899 & 0.0057 \\
\hline 5 & 2 & 4 & 4 & 2 & 3 & 7800.4618 & -0.0102 \\
\hline
\end{tabular}

Table S34. Measured frequencies and residuals (in $\mathrm{MHz}$ ) for the rotational transitions of the ${ }^{13} \mathrm{C}_{9}$ isotopologue of $\mathrm{BC} 1-1 \mathrm{w}-2$.

\begin{tabular}{rcccccrr}
\hline \hline $\mathrm{J}^{\prime}$ & $\mathrm{K}_{-1}^{\prime}$ & $\mathrm{K}^{\prime}{ }_{+1}$ & $\mathrm{~J}^{\prime \prime}$ & $\mathrm{K}_{-1}^{\prime \prime}$ & $\mathrm{K}^{\prime \prime}{ }_{+1}$ & $\mathrm{~V}_{\text {obs }}$ & $\mathrm{V}_{\text {obs }} \mathrm{V}_{\text {calc }}$ \\
\hline 2 & 0 & 2 & 1 & 0 & 1 & 3131.3981 & -0.0034 \\
2 & 1 & 1 & 1 & 1 & 0 & 3320.2323 & 0.0164 \\
3 & 1 & 3 & 2 & 1 & 2 & 4465.6753 & -0.0064 \\
3 & 0 & 3 & 2 & 0 & 2 & 4645.6053 & -0.0027 \\
3 & 2 & 1 & 2 & 2 & 0 & 4813.0597 & 0.0076 \\
3 & 1 & 2 & 2 & 1 & 1 & 4965.8979 & -0.0002 \\
4 & 1 & 4 & 3 & 1 & 3 & 5932.8541 & -0.0070 \\
4 & 0 & 4 & 3 & 0 & 3 & 6109.2457 & 0.0077 \\
4 & 2 & 3 & 3 & 2 & 2 & 6288.9589 & -0.0072 \\
4 & 1 & 3 & 3 & 1 & 2 & 6591.7883 & -0.0074 \\
5 & 1 & 5 & 4 & 1 & 4 & 7386.1723 & 0.0092 \\
5 & 2 & 4 & 4 & 2 & 3 & 7834.4548 & -0.0023 \\
\hline \hline
\end{tabular}


Table S35. Measured frequencies and residuals (in $\mathrm{MHz}$ ) for the rotational transitions of the ${ }^{18} \mathrm{O}_{10}$ isotopologue of $1 \mathrm{w}$-II.

\begin{tabular}{|c|c|c|c|c|c|c|c|}
\hline $\mathrm{J}^{\prime}$ & $\mathrm{K}_{-1}^{\prime}$ & $\mathrm{K}_{+1}^{\prime}$ & $\mathrm{J}^{\prime \prime}$ & $\mathrm{K}^{\prime \prime}{ }_{-1}$ & $\mathrm{~K}_{+1}^{\prime \prime}$ & $\mathrm{V}_{\mathrm{obs}}$ & $\mathrm{V}_{\text {obs }}-\mathrm{V}_{\text {calc }}$ \\
\hline 1 & 1 & 1 & 0 & 0 & 0 & 2459.1539 & -0.0149 \\
\hline 1 & 1 & 0 & 0 & 0 & 0 & 2610.6947 & 0.0025 \\
\hline 2 & 1 & 2 & 1 & 1 & 1 & 2881.1590 & -0.0042 \\
\hline 2 & 0 & 2 & 1 & 0 & 1 & 3015.8474 & -0.0034 \\
\hline 2 & 1 & 1 & 1 & 1 & 0 & 3184.2114 & 0.0018 \\
\hline 3 & 0 & 3 & 2 & 1 & 2 & 3674.8668 & -0.0084 \\
\hline 2 & 1 & 2 & 1 & 0 & 1 & 3823.9861 & 0.0000 \\
\hline 3 & 1 & 3 & 2 & 1 & 2 & 4311.7349 & -0.0016 \\
\hline 3 & 0 & 3 & 2 & 0 & 2 & 4483.0094 & -0.0012 \\
\hline 3 & 2 & 2 & 2 & 2 & 1 & 4549.0204 & 0.0032 \\
\hline 3 & 2 & 1 & 2 & 2 & 0 & 4615.0218 & 0.0002 \\
\hline 3 & 1 & 2 & 2 & 1 & 1 & 4765.1266 & -0.0005 \\
\hline 3 & 1 & 3 & 2 & 0 & 2 & 5119.8645 & -0.0074 \\
\hline 4 & 0 & 4 & 3 & 1 & 3 & 5271.3069 & 0.0068 \\
\hline 5 & 1 & 4 & 4 & 2 & 2 & 5300.3726 & -0.0016 \\
\hline 4 & 1 & 4 & 3 & 1 & 3 & 5731.8648 & 0.0003 \\
\hline 4 & 0 & 4 & 3 & 0 & 3 & 5908.1612 & -0.0002 \\
\hline 2 & 2 & 1 & 1 & 1 & 0 & 6012.6849 & 0.0170 \\
\hline 2 & 2 & 0 & 1 & 1 & 0 & 6029.5010 & -0.0022 \\
\hline 2 & 2 & 0 & 1 & 1 & 0 & 6029.5010 & -0.0022 \\
\hline 4 & 2 & 3 & 3 & 2 & 2 & 6052.1942 & -0.0045 \\
\hline 4 & 3 & 2 & 3 & 3 & 1 & 6095.7491 & -0.0032 \\
\hline 4 & 3 & 1 & 3 & 3 & 0 & 6102.7556 & -0.0055 \\
\hline 2 & 2 & 1 & 1 & 1 & 1 & 6164.1909 & -0.0004 \\
\hline 2 & 2 & 1 & 1 & 1 & 1 & 6164.1909 & -0.0004 \\
\hline 4 & 2 & 2 & 3 & 2 & 1 & 6209.2937 & -0.0022 \\
\hline 4 & 1 & 3 & 3 & 1 & 2 & 6330.9447 & 0.0000 \\
\hline 4 & 1 & 4 & 3 & 0 & 3 & 6368.7179 & -0.0079 \\
\hline 5 & 0 & 5 & 4 & 1 & 4 & 6833.0586 & 0.0099 \\
\hline 6 & 1 & 5 & 5 & 2 & 3 & 6853.2539 & 0.0020 \\
\hline 5 & 1 & 5 & 4 & 1 & 4 & 7140.4246 & 0.0117 \\
\hline 5 & 0 & 5 & 4 & 0 & 4 & 7293.6199 & 0.0068 \\
\hline 3 & 2 & 1 & 2 & 1 & 1 & 7460.3103 & -0.0049 \\
\hline 5 & 2 & 4 & 4 & 2 & 3 & 7544.2544 & -0.0024 \\
\hline 5 & 1 & 5 & 4 & 0 & 4 & 7600.9823 & 0.0050 \\
\hline 5 & 4 & 2 & 4 & 4 & 1 & 7618.9836 & -0.0110 \\
\hline 5 & 4 & 1 & 4 & 4 & 0 & 7619.5228 & -0.0150 \\
\hline 5 & 3 & 3 & 4 & 3 & 2 & 7628.1544 & -0.0078 \\
\hline 5 & 3 & 2 & 4 & 3 & 1 & 7652.2595 & -0.0062 \\
\hline 5 & 2 & 3 & 4 & 2 & 2 & 7831.7151 & -0.0016 \\
\hline 3 & 2 & 2 & 2 & 1 & 2 & 7832.0454 & 0.0001 \\
\hline 5 & 1 & 4 & 4 & 1 & 3 & 7873.9083 & -0.0052 \\
\hline
\end{tabular}


Table S36. Measured frequencies and residuals (in $\mathrm{MHz}$ ) for the rotational transitions of the parent species of $2 \mathrm{w}-\mathrm{I}$ complex.

\begin{tabular}{|c|c|c|c|c|c|c|c|}
\hline $\mathrm{J}^{\prime}$ & $\mathrm{K}^{\prime}{ }_{-1}$ & $\mathrm{~K}^{\prime}+1$ & J" & $\mathrm{K}^{\prime \prime}{ }_{-1}$ & $\mathrm{~K}^{\prime \prime}+1$ & $\mathrm{~V}_{\mathrm{obs}}$ & $\mathrm{V}_{\text {obs }}-\mathrm{V}_{\text {calc }}$ \\
\hline 2 & 1 & 2 & 1 & 1 & 1 & 2392.7120 & -0.0235 \\
\hline 2 & 0 & 2 & 1 & 0 & 1 & 2500.5824 & -0.0003 \\
\hline 2 & 1 & 1 & 1 & 1 & 0 & 2658.8923 & -0.0015 \\
\hline 2 & 1 & 1 & 1 & 0 & 1 & 3245.4508 & 0.0039 \\
\hline 3 & 1 & 3 & 2 & 1 & 2 & 3574.5796 & 0.0038 \\
\hline 3 & 0 & 3 & 2 & 0 & 2 & 3693.2505 & 0.0032 \\
\hline 3 & 2 & 2 & 2 & 2 & 1 & 3788.6926 & -0.0012 \\
\hline 3 & 2 & 1 & 2 & 2 & 0 & 3884.1627 & -0.0006 \\
\hline 3 & 1 & 2 & 2 & 1 & 1 & 3970.7554 & 0.0009 \\
\hline 2 & 2 & 0 & 1 & 1 & 0 & 4044.5512 & 0.0032 \\
\hline 2 & 2 & 1 & 1 & 1 & 1 & 4152.3981 & 0.0061 \\
\hline 4 & 1 & 4 & 3 & 1 & 3 & 4742.8412 & 0.0023 \\
\hline 4 & 0 & 4 & 3 & 0 & 3 & 4839.9886 & 0.0049 \\
\hline 4 & 2 & 3 & 3 & 2 & 2 & 5031.7774 & 0.0027 \\
\hline 4 & 3 & 2 & 3 & 3 & 1 & 5094.1409 & 0.0064 \\
\hline 4 & 3 & 1 & 3 & 3 & 0 & 5112.0191 & -0.0006 \\
\hline 4 & 2 & 2 & 3 & 2 & 1 & 5243.0870 & -0.0005 \\
\hline 4 & 1 & 3 & 3 & 1 & 2 & 5257.2636 & 0.0013 \\
\hline 3 & 2 & 1 & 2 & 1 & 1 & 5269.8060 & -0.0116 \\
\hline 3 & 2 & 1 & 2 & 1 & 1 & 5269.8060 & -0.0116 \\
\hline 3 & 2 & 2 & 2 & 1 & 2 & 5548.3510 & 0.0007 \\
\hline 5 & 1 & 5 & 4 & 1 & 4 & 5898.1537 & 0.0023 \\
\hline 5 & 0 & 5 & 4 & 0 & 4 & 5961.2674 & 0.0027 \\
\hline 5 & 2 & 4 & 4 & 2 & 3 & 6258.5437 & -0.0013 \\
\hline 4 & 1 & 3 & 3 & 0 & 3 & 6279.6296 & -0.0039 \\
\hline 5 & 4 & 2 & 4 & 4 & 1 & 6370.2078 & 0.0043 \\
\hline 5 & 4 & 1 & 4 & 4 & 0 & 6372.6106 & -0.0017 \\
\hline 5 & 3 & 3 & 4 & 3 & 2 & 6373.9817 & -0.0018 \\
\hline 3 & 3 & 0 & 2 & 2 & 0 & 6381.1011 & 0.0041 \\
\hline 3 & 3 & 1 & 2 & 2 & 1 & 6403.2737 & -0.0028 \\
\hline 5 & 3 & 2 & 4 & 3 & 1 & 6433.2286 & -0.0003 \\
\hline 5 & 1 & 4 & 4 & 1 & 3 & 6504.2581 & 0.0017 \\
\hline 4 & 2 & 2 & 3 & 1 & 2 & 6542.1546 & 0.0040 \\
\hline 5 & 2 & 3 & 4 & 2 & 2 & 6609.3408 & -0.0012 \\
\hline 4 & 2 & 3 & 3 & 1 & 3 & 7005.5524 & 0.0031 \\
\hline 6 & 1 & 6 & 5 & 1 & 5 & 7043.1698 & -0.0037 \\
\hline 6 & 0 & 6 & 5 & 0 & 5 & 7078.0521 & 0.0000 \\
\hline 6 & 2 & 5 & 5 & 2 & 4 & 7466.5003 & -0.0016 \\
\hline 4 & 3 & 1 & 3 & 2 & 1 & 7608.9524 & -0.0010 \\
\hline 6 & 3 & 4 & 5 & 3 & 3 & 7648.1195 & -0.0008 \\
\hline 6 & 4 & 3 & 5 & 4 & 2 & 7659.7128 & -0.0013 \\
\hline 6 & 4 & 2 & 5 & 4 & 1 & 7670.2407 & -0.0012 \\
\hline 6 & 1 & 5 & 5 & 1 & 4 & 7699.1476 & 0.0007 \\
\hline 4 & 3 & 2 & 3 & 2 & 2 & 7708.7170 & -0.0002 \\
\hline 6 & 3 & 3 & 5 & 3 & 2 & 7790.3162 & -0.0002 \\
\hline 5 & 2 & 3 & 4 & 1 & 3 & 7894.2351 & 0.0048 \\
\hline 6 & 2 & 4 & 5 & 2 & 3 & 7955.2363 & -0.0004 \\
\hline
\end{tabular}


Table S37. Measured frequencies and residuals (in $\mathrm{MHz}$ ) for the rotational transitions of the ${ }^{13} \mathrm{C}_{1}$ isotopologue of $2 \mathrm{w}-\mathrm{l}$.

\begin{tabular}{rcccccrr}
\hline \hline $\mathrm{J}^{\prime}$ & $\mathrm{K}_{-1}^{\prime}$ & $\mathrm{K}^{\prime}{ }_{+1}$ & $\mathrm{~J}^{\prime \prime}$ & $\mathrm{K}_{-1}^{\prime \prime}$ & $\mathrm{K}_{+1}^{\prime \prime}$ & $\mathrm{V}_{\text {obs }}$ & $\mathrm{V}_{\text {obs }}-\mathrm{V}_{\text {calc }}$ \\
\hline 3 & 1 & 3 & 2 & 1 & 2 & 3561.8104 & 0.0011 \\
3 & 0 & 3 & 2 & 0 & 2 & 3680.0562 & -0.0072 \\
3 & 1 & 2 & 2 & 1 & 1 & 3965.4273 & -0.0009 \\
4 & 1 & 4 & 3 & 1 & 3 & 4724.7007 & 0.0054 \\
4 & 0 & 4 & 3 & 0 & 3 & 4819.4619 & 0.0013 \\
4 & 2 & 3 & 3 & 2 & 2 & 5019.8283 & 0.0088 \\
4 & 2 & 2 & 3 & 2 & 1 & 5240.8045 & -0.0040 \\
4 & 1 & 3 & 3 & 1 & 2 & 5247.7211 & 0.0018 \\
5 & 0 & 5 & 4 & 0 & 4 & 5934.3139 & 0.0102 \\
5 & 1 & 4 & 4 & 1 & 3 & 6487.8476 & -0.0032 \\
6 & 1 & 6 & 5 & 1 & 5 & 7013.4139 & -0.0040 \\
6 & 0 & 6 & 5 & 0 & 5 & 7045.7782 & -0.0095 \\
6 & 2 & 5 & 5 & 2 & 4 & 7444.1496 & 0.0028 \\
6 & 1 & 5 & 5 & 1 & 4 & 7673.1762 & -0.0006 \\
\hline \hline
\end{tabular}

Table S38. Measured frequencies and residuals (in $\mathrm{MHz}$ ) for the rotational transitions of the ${ }^{13} \mathrm{C}_{2}$ isotopologue of $2 \mathrm{w}-\mathrm{l}$.

\begin{tabular}{rcccccrr}
\hline \hline $\mathrm{J}^{\prime}$ & $\mathrm{K}_{-1}^{\prime}$ & $\mathrm{K}^{\prime}{ }_{+1}$ & $\mathrm{~J}^{\prime \prime}$ & $\mathrm{K}_{-1}^{\prime \prime}$ & $\mathrm{K}^{\prime \prime}{ }_{+1}$ & $\mathrm{~V}_{\text {obs }}$ & $\mathrm{V}_{\text {obs }}-\mathrm{V}_{\text {calc }}$ \\
\hline 3 & 1 & 3 & 2 & 1 & 2 & 3564.8964 & 0.0081 \\
3 & 0 & 3 & 2 & 0 & 2 & 3682.6010 & 0.0000 \\
3 & 1 & 2 & 2 & 1 & 1 & 3959.4798 & -0.0024 \\
4 & 1 & 4 & 3 & 1 & 3 & 4729.8903 & 0.0017 \\
4 & 0 & 4 & 3 & 0 & 3 & 4825.8655 & -0.0026 \\
4 & 1 & 3 & 3 & 1 & 2 & 5242.0640 & -0.0014 \\
5 & 1 & 5 & 4 & 1 & 4 & 5881.9738 & 0.0104 \\
5 & 0 & 5 & 4 & 0 & 4 & 5944.0257 & -0.0037 \\
5 & 2 & 4 & 4 & 2 & 3 & 6240.9950 & -0.0026 \\
5 & 1 & 4 & 4 & 1 & 3 & 6484.9404 & -0.0056 \\
6 & 0 & 6 & 5 & 0 & 5 & 7057.9322 & -0.0103 \\
6 & 1 & 5 & 5 & 1 & 4 & 7675.6164 & 0.0101 \\
\hline \hline
\end{tabular}

Table S39. Measured frequencies and residuals (in $\mathrm{MHz}$ ) for the rotational transitions of the ${ }^{13} \mathrm{C}_{3}$ isotopologue of $2 \mathrm{w}-\mathrm{l}$.

\begin{tabular}{rccccccr}
\hline \hline $\mathrm{J}^{\prime}$ & $\mathrm{K}_{-1}^{\prime}$ & $\mathrm{K}^{\prime}{ }_{+1}$ & $\mathrm{~J}^{\prime \prime}$ & $\mathrm{K}_{-1}^{\prime \prime}$ & $\mathrm{K}^{\prime \prime}{ }_{+1}$ & $\mathrm{~V}_{\text {obs }}$ & $\mathrm{V}_{\text {obs }}-\mathrm{V}_{\text {calc }}$ \\
\hline 3 & 0 & 3 & 2 & 0 & 2 & 3666.9639 & -0.0039 \\
3 & 1 & 2 & 2 & 1 & 1 & 3938.1549 & -0.0080 \\
4 & 1 & 4 & 3 & 1 & 3 & 4709.5550 & -0.0016 \\
4 & 0 & 4 & 3 & 0 & 3 & 4807.1838 & -0.0078 \\
4 & 2 & 3 & 3 & 2 & 2 & 4992.9592 & 0.0055 \\
4 & 1 & 3 & 3 & 1 & 2 & 5215.3479 & -0.0046 \\
5 & 1 & 5 & 4 & 1 & 4 & 5857.4275 & -0.0014 \\
5 & 0 & 5 & 4 & 0 & 4 & 5921.7271 & 0.0119 \\
5 & 1 & 4 & 4 & 1 & 3 & 6454.6956 & -0.0094 \\
5 & 2 & 3 & 4 & 2 & 2 & 6552.2461 & 0.0053 \\
6 & 1 & 6 & 5 & 1 & 5 & 6995.1499 & -0.0018 \\
6 & 2 & 5 & 5 & 2 & 4 & 7411.1991 & -0.0044 \\
6 & 1 & 5 & 5 & 1 & 4 & 7643.8199 & 0.0090 \\
6 & 2 & 4 & 5 & 2 & 3 & 7888.2714 & 0.0035 \\
\hline \hline
\end{tabular}


Table S40. Measured frequencies and residuals (in $\mathrm{MHz}$ ) for the rotational transitions of the ${ }^{13} \mathrm{C}_{4}$ isotopologue of $2 \mathrm{w}-\mathrm{l}$.

\begin{tabular}{|c|c|c|c|c|c|c|c|}
\hline 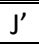 & $\mathrm{K}^{\prime}{ }_{-1}$ & $\mathrm{~K}_{+1}^{\prime}$ & $\mathrm{J}^{\prime \prime}$ & $\mathrm{K}^{\prime \prime}{ }_{-1}$ & $\mathrm{~K}_{+1}^{\prime \prime}$ & $\mathrm{V}_{\text {obs }}$ & $\mathrm{V}_{\text {obs }}-\mathrm{V}_{\text {calc }}$ \\
\hline 3 & 1 & 3 & 2 & 1 & 2 & 3547.2180 & 0.0031 \\
\hline 3 & 0 & 3 & 2 & 0 & 2 & 3665.3999 & -0.0029 \\
\hline 3 & 1 & 2 & 2 & 1 & 1 & 3937.3001 & -0.0074 \\
\hline 4 & 1 & 4 & 3 & 1 & 3 & 4707.0508 & -0.0101 \\
\hline 4 & 0 & 4 & 3 & 0 & 3 & 4804.8836 & 0.0025 \\
\hline 4 & 2 & 3 & 3 & 2 & 2 & 4991.1865 & 0.0136 \\
\hline 4 & 2 & 2 & 3 & 2 & 1 & 5196.3248 & 0.0074 \\
\hline 4 & 1 & 3 & 3 & 1 & 2 & 5214.0978 & -0.0032 \\
\hline 5 & 1 & 5 & 4 & 1 & 4 & 5854.2228 & -0.0044 \\
\hline 5 & 0 & 5 & 4 & 0 & 4 & 5918.6202 & 0.0070 \\
\hline 5 & 2 & 4 & 4 & 2 & 3 & 6208.8595 & 0.0027 \\
\hline 5 & 1 & 4 & 4 & 1 & 3 & 6452.9366 & -0.0017 \\
\hline 5 & 2 & 3 & 4 & 2 & 2 & 6550.8616 & -0.0022 \\
\hline 6 & 1 & 6 & 5 & 1 & 5 & 6991.2158 & -0.0038 \\
\hline 6 & 2 & 5 & 5 & 2 & 4 & 7408.3025 & 0.0030 \\
\hline 6 & 2 & 4 & 5 & 2 & 3 & 7886.6249 & -0.0035 \\
\hline
\end{tabular}

Table S41. Measured frequencies and residuals (in $\mathrm{MHz}$ ) for the rotational transitions of the ${ }^{13} \mathrm{C}_{5}$ isotopologue of $2 \mathrm{w}-\mathrm{l}$.

\begin{tabular}{rcccccrr}
\hline \hline $\mathrm{J}^{\prime}$ & $\mathrm{K}_{-1}^{\prime}$ & $\mathrm{K}^{\prime}{ }_{+1}$ & $\mathrm{~J}^{\prime \prime}$ & $\mathrm{K}^{\prime \prime}{ }_{-1}$ & $\mathrm{~K}^{\prime \prime}{ }_{+1}$ & $\mathrm{~V}_{\mathrm{obs}}$ & $\mathrm{V}_{\text {obs }} \mathrm{V}_{\text {calc }}$ \\
\hline 3 & 1 & 3 & 2 & 1 & 2 & 3555.1211 & 0.0037 \\
3 & 0 & 3 & 2 & 0 & 2 & 3673.4030 & 0.0092 \\
3 & 1 & 2 & 2 & 1 & 1 & 3950.0596 & 0.0010 \\
4 & 1 & 4 & 3 & 1 & 3 & 4716.9464 & -0.0130 \\
4 & 0 & 4 & 3 & 0 & 3 & 4813.7577 & -0.0039 \\
4 & 2 & 3 & 3 & 2 & 2 & 5005.0088 & 0.0086 \\
4 & 2 & 2 & 3 & 2 & 1 & 5215.7006 & -0.0076 \\
4 & 1 & 3 & 3 & 1 & 2 & 5229.7616 & -0.0064 \\
5 & 1 & 5 & 4 & 1 & 4 & 5865.8995 & 0.0109 \\
5 & 0 & 5 & 4 & 0 & 4 & 5928.7535 & -0.0110 \\
5 & 2 & 4 & 4 & 2 & 3 & 6225.1557 & -0.0066 \\
5 & 1 & 4 & 4 & 1 & 3 & 6470.0715 & 0.0027 \\
5 & 2 & 3 & 4 & 2 & 2 & 6574.9400 & 0.0096 \\
6 & 1 & 6 & 5 & 1 & 5 & 7004.5545 & -0.0042 \\
6 & 0 & 6 & 5 & 0 & 5 & 7039.3067 & 0.0090 \\
6 & 2 & 5 & 5 & 2 & 4 & 7426.5639 & 0.0007 \\
6 & 1 & 5 & 5 & 1 & 4 & 7658.4113 & 0.0013 \\
6 & 2 & 4 & 5 & 2 & 3 & 7913.8302 & -0.0035 \\
\hline \hline
\end{tabular}


Table S42. Measured frequencies and residuals (in $\mathrm{MHz}$ ) for the rotational transitions of the ${ }^{13} \mathrm{C}_{6}$ isotopologue of $2 \mathrm{w}-\mathrm{l}$.

\begin{tabular}{rcccccrr}
\hline \hline $\mathrm{J}^{\prime}$ & $\mathrm{K}_{-1}^{\prime}$ & $\mathrm{K}_{+1}^{\prime}$ & $\mathrm{J}^{\prime \prime}$ & $\mathrm{K}_{-1}^{\prime \prime}$ & $\mathrm{K}^{\prime \prime}{ }_{+1}$ & \multicolumn{1}{c}{$\mathrm{V}_{\text {obs }}$} & $\mathrm{V}_{\text {obs }} \mathrm{V}_{\text {calc }}$ \\
\hline 3 & 1 & 3 & 2 & 1 & 2 & 3569.8550 & 0.0029 \\
3 & 0 & 3 & 2 & 0 & 2 & 3688.1673 & -0.0021 \\
3 & 1 & 2 & 2 & 1 & 1 & 3967.1466 & 0.0034 \\
4 & 1 & 4 & 3 & 1 & 3 & 4736.2942 & -0.0020 \\
4 & 0 & 4 & 3 & 0 & 3 & 4832.6173 & 0.0055 \\
4 & 2 & 2 & 3 & 2 & 1 & 5239.6377 & -0.0036 \\
4 & 1 & 3 & 3 & 1 & 2 & 5251.9005 & 0.0059 \\
5 & 1 & 5 & 4 & 1 & 4 & 5889.7158 & -0.0003 \\
5 & 0 & 5 & 4 & 0 & 4 & 5951.8777 & -0.0026 \\
5 & 2 & 4 & 4 & 2 & 3 & 6251.2478 & 0.0102 \\
5 & 1 & 4 & 4 & 1 & 3 & 6496.5308 & 0.0018 \\
5 & 2 & 3 & 4 & 2 & 2 & 6604.7441 & -0.0019 \\
6 & 2 & 5 & 5 & 2 & 4 & 7457.2231 & 0.0025 \\
6 & 1 & 5 & 5 & 1 & 4 & 7688.4481 & -0.0059 \\
\hline \hline
\end{tabular}

Table S43. Measured frequencies and residuals (in $\mathrm{MHz}$ ) for the rotational transitions of the ${ }^{18} \mathrm{O}_{7}$ isotopologue of $2 \mathrm{w}-\mathrm{I}$.

\begin{tabular}{rrrrrrrr}
\hline \hline$J^{\prime}$ & $\mathrm{K}_{-1}^{\prime}$ & $\mathrm{K}^{\prime}{ }_{+1}$ & $\mathrm{~J}^{\prime \prime}$ & $\mathrm{K}^{\prime \prime}{ }_{-1}$ & $\mathrm{~K}^{\prime \prime}{ }_{1}$ & $\mathrm{~V}_{\text {obs }}$ & $\mathrm{V}_{\text {obs }}-\mathrm{V}_{\text {calc }}$ \\
\hline 2 & 1 & 2 & 1 & 1 & 1 & 2378.4060 & 0.0038 \\
2 & 0 & 2 & 1 & 0 & 1 & 2488.1759 & -0.0033 \\
2 & 1 & 1 & 1 & 1 & 0 & 2653.5432 & -0.0035 \\
3 & 1 & 3 & 2 & 1 & 2 & 3551.6762 & 0.0017 \\
3 & 0 & 3 & 2 & 0 & 2 & 3669.5187 & -0.0022 \\
3 & 1 & 2 & 2 & 1 & 1 & 3960.7939 & 0.0024 \\
4 & 1 & 4 & 3 & 1 & 3 & 4710.3297 & -0.0006 \\
4 & 0 & 4 & 3 & 0 & 3 & 4803.2448 & -0.0035 \\
4 & 2 & 3 & 3 & 2 & 2 & 5010.0570 & 0.0010 \\
4 & 2 & 2 & 3 & 2 & 1 & 5238.3419 & -0.0040 \\
4 & 1 & 3 & 3 & 1 & 2 & 5239.6414 & 0.0072 \\
5 & 1 & 5 & 4 & 1 & 4 & 5855.3917 & 0.0048 \\
5 & 0 & 5 & 4 & 0 & 4 & 5913.1675 & -0.0002 \\
5 & 2 & 4 & 4 & 2 & 3 & 6228.2954 & 0.0047 \\
5 & 1 & 4 & 4 & 1 & 3 & 6474.2694 & -0.0026 \\
5 & 2 & 3 & 4 & 2 & 2 & 6601.9702 & -0.0018 \\
6 & 1 & 6 & 5 & 1 & 5 & 6989.9872 & -0.0004 \\
6 & 0 & 6 & 5 & 0 & 5 & 7020.5529 & -0.0057 \\
6 & 2 & 5 & 5 & 2 & 4 & 7426.1176 & 0.0033 \\
6 & 2 & 4 & 5 & 2 & 3 & 7940.1960 & -0.0013 \\
\hline \hline
\end{tabular}


Table S44. Measured frequencies and residuals (in $\mathrm{MHz}$ ) for the rotational transitions of the ${ }^{13} \mathrm{C}_{8}$ isotopologue of $2 \mathrm{w}-\mathrm{l}$.

\begin{tabular}{rcccccrr}
\hline \hline $\mathrm{J}^{\prime}$ & $\mathrm{K}_{-1}^{\prime}$ & $\mathrm{K}^{\prime}{ }_{+1}$ & $\mathrm{~J}^{\prime \prime}$ & $\mathrm{K}^{\prime \prime}{ }_{-1}$ & $\mathrm{~K}^{\prime \prime}{ }_{+1}$ & $\mathrm{~V}_{\text {obs }}$ & $\mathrm{V}_{\text {obs }}-\mathrm{V}_{\text {calc }}$ \\
\hline 3 & 1 & 3 & 2 & 1 & 2 & 3565.8640 & 0.0001 \\
3 & 0 & 3 & 2 & 0 & 2 & 3683.1428 & -0.0046 \\
3 & 1 & 2 & 2 & 1 & 1 & 3959.4798 & 0.0054 \\
4 & 1 & 4 & 3 & 1 & 3 & 4731.2153 & 0.0029 \\
4 & 0 & 4 & 3 & 0 & 3 & 4826.7307 & -0.0045 \\
4 & 1 & 3 & 3 & 1 & 2 & 5242.0640 & 0.0052 \\
5 & 1 & 5 & 4 & 1 & 4 & 5883.6596 & -0.0007 \\
5 & 0 & 5 & 4 & 0 & 4 & 5945.3552 & 0.0053 \\
5 & 2 & 4 & 4 & 2 & 3 & 6241.8131 & -0.0090 \\
5 & 1 & 4 & 4 & 1 & 3 & 6484.9404 & -0.0018 \\
6 & 0 & 6 & 5 & 0 & 5 & 7059.7931 & 0.0022 \\
\hline \hline
\end{tabular}

Table S45. Measured frequencies and residuals (in $\mathrm{MHz}$ ) for the rotational transitions of the ${ }^{13} \mathrm{C}_{9}$ isotopologue of $2 \mathrm{w}-\mathrm{I}$.

\begin{tabular}{rccccccr}
\hline \hline $\mathrm{J}^{\prime}$ & $\mathrm{K}_{-1}^{\prime}$ & $\mathrm{K}^{\prime}+1$ & $\mathrm{~J}^{\prime \prime}$ & $\mathrm{K}_{-1}^{\prime}$ & $\mathrm{K}^{\prime \prime}{ }_{+1}$ & $\mathrm{~V}_{\text {obs }}$ & $\mathrm{V}_{\text {obs }} \mathrm{V}_{\text {calc }}$ \\
\hline 3 & 1 & 3 & 2 & 1 & 2 & 3571.4938 & -0.0069 \\
3 & 0 & 3 & 2 & 0 & 2 & 3688.8410 & 0.0034 \\
3 & 1 & 2 & 2 & 1 & 1 & 3961.5687 & -0.0088 \\
4 & 1 & 4 & 3 & 1 & 3 & 4739.2245 & 0.0012 \\
4 & 0 & 4 & 3 & 0 & 3 & 4835.6711 & 0.0041 \\
4 & 2 & 3 & 3 & 2 & 2 & 5023.5691 & 0.0018 \\
4 & 2 & 2 & 3 & 2 & 1 & 5230.5556 & 0.0020 \\
4 & 1 & 3 & 3 & 1 & 2 & 5245.9212 & 0.0039 \\
5 & 1 & 5 & 4 & 1 & 4 & 5894.2225 & 0.0026 \\
5 & 0 & 5 & 4 & 0 & 4 & 5957.1754 & -0.0039 \\
5 & 2 & 4 & 4 & 2 & 3 & 6248.9789 & -0.0019 \\
5 & 1 & 4 & 4 & 1 & 3 & 6491.7038 & 0.0014 \\
6 & 1 & 5 & 5 & 1 & 4 & 7686.5148 & -0.0011 \\
\hline \hline
\end{tabular}


Table S46. Measured frequencies and residuals (in $\mathrm{MHz}$ ) for the rotational transitions of the ${ }^{18} \mathrm{O}_{10}$ isotopologue of $2 \mathrm{w}-\mathrm{I}$.

\begin{tabular}{|c|c|c|c|c|c|c|c|}
\hline $\mathrm{J}^{\prime}$ & $\mathrm{K}_{-1}^{\prime}$ & $\mathrm{K}_{+1}^{\prime}$ & J" & $\mathrm{K}_{-1}^{\prime \prime}$ & $\mathrm{K}^{\prime \prime}+1$ & $\mathrm{~V}_{\mathrm{obs}}$ & $\mathrm{V}_{\text {obs }}-\mathrm{V}_{\text {calc }}$ \\
\hline 2 & 1 & 2 & 1 & 1 & 1 & 2334.1094 & 0.0009 \\
\hline 2 & 0 & 2 & 1 & 0 & 1 & 2439.2744 & 0.0016 \\
\hline 2 & 1 & 1 & 1 & 1 & 0 & 2591.1848 & 0.0018 \\
\hline 3 & 1 & 3 & 2 & 1 & 2 & 3487.6668 & 0.0021 \\
\hline 3 & 0 & 3 & 2 & 0 & 2 & 3605.1543 & -0.0005 \\
\hline 3 & 2 & 2 & 2 & 2 & 1 & 3693.9449 & 0.0046 \\
\hline 3 & 2 & 1 & 2 & 2 & 0 & 3782.7511 & 0.0016 \\
\hline 3 & 1 & 2 & 2 & 1 & 1 & 3870.5661 & 0.0017 \\
\hline 2 & 2 & 1 & 1 & 1 & 1 & 4099.5435 & 0.0070 \\
\hline 4 & 1 & 4 & 3 & 1 & 3 & 4628.4628 & 0.0014 \\
\hline 4 & 0 & 4 & 3 & 0 & 3 & 4726.9071 & -0.0006 \\
\hline 4 & 2 & 3 & 3 & 2 & 2 & 4906.9074 & -0.0004 \\
\hline 4 & 3 & 2 & 3 & 3 & 1 & 4964.9180 & -0.0004 \\
\hline 4 & 3 & 1 & 3 & 3 & 0 & 4980.8143 & -0.0004 \\
\hline 4 & 2 & 2 & 3 & 2 & 1 & 5105.0069 & 0.0011 \\
\hline 4 & 1 & 3 & 3 & 1 & 2 & 5126.7103 & 0.0007 \\
\hline 3 & 2 & 1 & 2 & 1 & 1 & 5185.9335 & -0.0088 \\
\hline 3 & 2 & 2 & 2 & 1 & 2 & 5459.3650 & -0.0032 \\
\hline 5 & 1 & 5 & 4 & 1 & 4 & 5756.8976 & -0.0005 \\
\hline 5 & 0 & 5 & 4 & 0 & 4 & 5822.6540 & 0.0003 \\
\hline 5 & 2 & 4 & 4 & 2 & 3 & 6104.7260 & 0.0010 \\
\hline 4 & 1 & 3 & 3 & 0 & 3 & 6132.5088 & 0.0017 \\
\hline 5 & 4 & 2 & 4 & 4 & 1 & 6208.1741 & -0.0035 \\
\hline 5 & 4 & 1 & 4 & 4 & 0 & 6210.2195 & -0.0068 \\
\hline 5 & 3 & 3 & 4 & 3 & 2 & 6212.5393 & -0.0010 \\
\hline 5 & 3 & 2 & 4 & 3 & 1 & 6265.4500 & -0.0016 \\
\hline 3 & 3 & 0 & 2 & 2 & 0 & 6307.7971 & 0.0044 \\
\hline 3 & 3 & 1 & 2 & 2 & 1 & 6328.4581 & -0.0009 \\
\hline 5 & 1 & 4 & 4 & 1 & 3 & 6346.6422 & 0.0016 \\
\hline 5 & 2 & 3 & 4 & 2 & 2 & 6436.5756 & 0.0029 \\
\hline 6 & 1 & 6 & 5 & 1 & 5 & 6875.3071 & -0.0026 \\
\hline 4 & 2 & 3 & 3 & 1 & 3 & 6878.6154 & 0.0040 \\
\hline 6 & 0 & 6 & 5 & 0 & 5 & 6912.6759 & 0.0001 \\
\hline 6 & 2 & 5 & 5 & 2 & 4 & 7284.9572 & 0.0027 \\
\hline 6 & 3 & 4 & 5 & 3 & 3 & 7455.3691 & -0.0010 \\
\hline 6 & 4 & 3 & 5 & 4 & 2 & 7464.3520 & -0.0043 \\
\hline 6 & 4 & 2 & 5 & 4 & 1 & 7473.3283 & -0.0048 \\
\hline 6 & 1 & 5 & 5 & 1 & 4 & 7518.1465 & 0.0073 \\
\hline 6 & 3 & 3 & 5 & 3 & 2 & 7583.5249 & 0.0016 \\
\hline 4 & 3 & 2 & 3 & 2 & 2 & 7599.4356 & -0.0016 \\
\hline 5 & 2 & 3 & 4 & 1 & 3 & 7730.2394 & -0.0074 \\
\hline 6 & 2 & 4 & 5 & 2 & 3 & 7751.1188 & 0.0053 \\
\hline
\end{tabular}


Table S47. Measured frequencies and residuals (in $\mathrm{MHz}$ ) for the rotational transitions of the ${ }^{18} \mathrm{O}_{11}$ isotopologue of $2 \mathrm{w}-\mathrm{l}$.

\begin{tabular}{|c|c|c|c|c|c|c|c|}
\hline $\mathrm{J}^{\prime}$ & $\mathrm{K}^{\prime}{ }_{-1}$ & $\mathrm{~K}_{+1}^{\prime}$ & $\mathrm{J}^{\prime \prime}$ & $\mathrm{K}^{\prime \prime}{ }_{-1}$ & $\mathrm{~K}_{+1}^{\prime \prime}$ & $V_{\text {obs }}$ & $\mathrm{V}_{\text {obs }}-\mathrm{V}_{\text {calc }}$ \\
\hline 2 & 1 & 2 & 1 & 1 & 1 & 2331.3494 & 0.0012 \\
\hline 2 & 0 & 2 & 1 & 0 & 1 & 2436.8461 & -0.0003 \\
\hline 2 & 1 & 1 & 1 & 1 & 0 & 2589.1186 & -0.0003 \\
\hline 3 & 1 & 3 & 2 & 1 & 2 & 3483.5141 & 0.0000 \\
\hline 3 & 0 & 3 & 2 & 0 & 2 & 3601.4630 & -0.0015 \\
\hline 3 & 2 & 2 & 2 & 2 & 1 & 3690.3151 & -0.0069 \\
\hline 3 & 2 & 1 & 2 & 2 & 0 & 3779.2037 & 0.0006 \\
\hline 3 & 1 & 2 & 2 & 1 & 1 & 3867.4621 & -0.0002 \\
\hline 2 & 2 & 0 & 1 & 1 & 0 & 3999.4447 & -0.0017 \\
\hline 2 & 2 & 1 & 1 & 1 & 1 & 4104.9506 & 0.0097 \\
\hline 4 & 1 & 4 & 3 & 1 & 3 & 4622.9034 & -0.0004 \\
\hline 4 & 0 & 4 & 3 & 0 & 3 & 4721.8470 & -0.0019 \\
\hline 4 & 2 & 3 & 3 & 2 & 2 & 4902.0718 & -0.0011 \\
\hline 4 & 3 & 2 & 3 & 3 & 1 & 4960.1360 & 0.0050 \\
\hline 4 & 3 & 1 & 3 & 3 & 0 & 4976.0080 & 0.0041 \\
\hline 4 & 2 & 2 & 3 & 2 & 1 & 5100.4040 & -0.0023 \\
\hline 4 & 1 & 3 & 3 & 1 & 2 & 5122.5659 & -0.0018 \\
\hline 3 & 2 & 1 & 2 & 1 & 1 & 5189.5275 & -0.0031 \\
\hline 5 & 1 & 5 & 4 & 1 & 4 & 5749.9057 & -0.0025 \\
\hline 5 & 0 & 5 & 4 & 0 & 4 & 5816.0867 & -0.0006 \\
\hline 5 & 2 & 4 & 4 & 2 & 3 & 6098.6617 & -0.0007 \\
\hline 4 & 1 & 3 & 3 & 0 & 3 & 6132.0376 & 0.0004 \\
\hline 5 & 4 & 2 & 4 & 4 & 1 & 6202.1856 & 0.0064 \\
\hline 5 & 4 & 1 & 4 & 4 & 0 & 6204.2244 & 0.0040 \\
\hline 5 & 3 & 3 & 4 & 3 & 2 & 6206.5891 & 0.0023 \\
\hline 3 & 3 & 0 & 2 & 2 & 0 & 6316.9083 & 0.0045 \\
\hline 3 & 3 & 1 & 2 & 2 & 1 & 6337.5906 & 0.0020 \\
\hline 5 & 1 & 4 & 4 & 1 & 3 & 6341.4545 & -0.0023 \\
\hline 4 & 2 & 2 & 3 & 1 & 2 & 6422.4708 & -0.0038 \\
\hline 5 & 2 & 3 & 4 & 2 & 2 & 6431.0493 & -0.0042 \\
\hline 6 & 1 & 6 & 5 & 1 & 5 & 6866.8575 & 0.0008 \\
\hline 6 & 0 & 6 & 5 & 0 & 5 & 6904.5157 & 0.0001 \\
\hline 6 & 2 & 5 & 5 & 2 & 4 & 7277.6441 & -0.0018 \\
\hline 6 & 3 & 4 & 5 & 3 & 3 & 7448.2729 & 0.0000 \\
\hline 6 & 4 & 3 & 5 & 4 & 2 & 7457.1826 & 0.0052 \\
\hline 6 & 4 & 2 & 5 & 4 & 1 & 7466.1302 & 0.0078 \\
\hline 6 & 1 & 5 & 5 & 1 & 4 & 7511.8764 & -0.0029 \\
\hline 4 & 3 & 1 & 3 & 2 & 1 & 7513.7007 & -0.0039 \\
\hline 6 & 3 & 3 & 5 & 3 & 2 & 7576.3214 & -0.0014 \\
\hline 4 & 3 & 2 & 3 & 2 & 2 & 7607.3961 & -0.0015 \\
\hline 5 & 2 & 3 & 4 & 1 & 3 & 7730.9579 & -0.0025 \\
\hline 6 & 2 & 4 & 5 & 2 & 3 & 7744.7553 & -0.0051 \\
\hline 7 & 1 & 7 & 6 & 1 & 6 & 7976.7878 & 0.0014 \\
\hline
\end{tabular}


Table S48. Measured frequencies and residuals (in $\mathrm{MHz}$ ) for the rotational transitions of the parent species of $2 \mathrm{w}$-IV complex.

\begin{tabular}{|c|c|c|c|c|c|c|c|}
\hline$\overline{J^{\prime}}$ & $\overline{\mathrm{K}^{\prime}-1}$ & $\overline{\mathrm{K}^{\prime}+1}$ & $\overline{\mathrm{J \prime \prime}}$ & $\mathrm{K}_{-1}^{\prime \prime}$ & $\mathrm{K}^{\prime \prime}{ }_{+1}$ & V & $\begin{array}{l}V_{\text {obs }}-V_{\text {calc }} \\
\end{array}$ \\
\hline 2 & 0 & 2 & 1 & 0 & 1 & 2152.3842 & -0.0047 \\
\hline 2 & 1 & 1 & 1 & 1 & 0 & 2229.5157 & 0.0046 \\
\hline 3 & 1 & 3 & 2 & 1 & 2 & 3123.4773 & -0.0009 \\
\hline 3 & 0 & 3 & 2 & 0 & 2 & 3217.6225 & -0.0017 \\
\hline 3 & 2 & 2 & 2 & 2 & 1 & 3235.2169 & 0.0105 \\
\hline 3 & 2 & 1 & 2 & 2 & 0 & 3252.7907 & -0.0063 \\
\hline 3 & 1 & 2 & 2 & 1 & 1 & 3341.4074 & 0.0009 \\
\hline 4 & 1 & 4 & 3 & 1 & 3 & 4159.8417 & -0.0022 \\
\hline 4 & 0 & 4 & 3 & 0 & 3 & 4270.3164 & -0.0009 \\
\hline 4 & 2 & 3 & 3 & 2 & 2 & 4310.1545 & 0.0007 \\
\hline 4 & 3 & 2 & 3 & 3 & 1 & 4321.9492 & 0.0000 \\
\hline 4 & 3 & 1 & 3 & 3 & 0 & 4322.9528 & -0.0061 \\
\hline 4 & 2 & 2 & 3 & 2 & 1 & 4353.4389 & -0.0019 \\
\hline 4 & 1 & 3 & 3 & 1 & 2 & 4449.6302 & 0.0011 \\
\hline 2 & 2 & 0 & 1 & 1 & 0 & 4809.1292 & -0.0059 \\
\hline 5 & 1 & 5 & 4 & 1 & 4 & 5192.5728 & -0.0036 \\
\hline 5 & 0 & 5 & 4 & 0 & 4 & 5308.0590 & -0.0004 \\
\hline 5 & 2 & 4 & 4 & 2 & 3 & 5382.1550 & 0.0029 \\
\hline 5 & 4 & 1 & 4 & 4 & 0 & 5401.8024 & -0.0091 \\
\hline 5 & 3 & 3 & 4 & 3 & 2 & 5405.4787 & 0.0014 \\
\hline 5 & 3 & 2 & 4 & 3 & 1 & 5408.9920 & -0.0013 \\
\hline 5 & 2 & 3 & 4 & 2 & 2 & 5466.0411 & -0.0048 \\
\hline 5 & 1 & 4 & 4 & 1 & 3 & 5552.5559 & 0.0006 \\
\hline 4 & 1 & 3 & 3 & 0 & 3 & 5581.6293 & -0.0057 \\
\hline 6 & 1 & 6 & 5 & 1 & 5 & 6221.3116 & -0.0041 \\
\hline 6 & 0 & 6 & 5 & 0 & 5 & 6330.8285 & -0.0031 \\
\hline 6 & 2 & 5 & 5 & 2 & 4 & 6450.4995 & 0.0050 \\
\hline 6 & 5 & 1 & 5 & 5 & 0 & 6481.3498 & 0.0027 \\
\hline 6 & 4 & 3 & 5 & 4 & 2 & 6485.2857 & -0.0019 \\
\hline 6 & 4 & 2 & 5 & 4 & 1 & 6485.4879 & 0.0092 \\
\hline 6 & 3 & 4 & 5 & 3 & 3 & 6490.2072 & 0.0006 \\
\hline 6 & 3 & 3 & 5 & 3 & 2 & 6499.4926 & -0.0028 \\
\hline 6 & 2 & 4 & 5 & 2 & 3 & 6589.8401 & -0.0026 \\
\hline 6 & 1 & 5 & 5 & 1 & 4 & 6648.2220 & 0.0026 \\
\hline 4 & 2 & 2 & 3 & 1 & 2 & 6844.4620 & 0.0068 \\
\hline 5 & 1 & 4 & 4 & 0 & 4 & 6863.8740 & 0.0011 \\
\hline 7 & 1 & 7 & 6 & 1 & 6 & 7245.9891 & 0.0026 \\
\hline 7 & 0 & 7 & 6 & 0 & 6 & 7341.2977 & -0.0039 \\
\hline 7 & 2 & 6 & 6 & 2 & 5 & 7514.5277 & 0.0100 \\
\hline 7 & 6 & 2 & 6 & 6 & 1 & 7560.9671 & 0.0011 \\
\hline 7 & 5 & 2 & 6 & 5 & 1 & 7564.3433 & -0.0036 \\
\hline 7 & 3 & 5 & 6 & 3 & 4 & 7575.6561 & 0.0025 \\
\hline 7 & 3 & 4 & 6 & 3 & 3 & 7596.2236 & -0.0032 \\
\hline 7 & 2 & 5 & 6 & 2 & 4 & 7721.3060 & -0.0089 \\
\hline 7 & 1 & 6 & 6 & 1 & 5 & 7734.3006 & 0.0027 \\
\hline
\end{tabular}


Table S49. Measured frequencies and residuals (in MHz) for the rotational transitions of the ${ }^{13} \mathrm{C}_{1}$ isotopologue of $2 \mathrm{w}-\mathrm{IV}$.

\begin{tabular}{|c|c|c|c|c|c|c|c|}
\hline$\overline{J^{\prime}}$ & $\mathrm{K}^{\prime}{ }_{-1}$ & $\mathrm{~K}^{\prime}+1$ & $\mathrm{~J}^{\prime \prime}$ & $\mathrm{K}^{\prime \prime}{ }_{-1}$ & $\mathrm{~K}_{+1}^{\prime \prime}$ & $\mathrm{V}_{\text {obs }}$ & $\mathrm{V}_{\text {obs }}-\mathrm{V}_{\text {calc }}$ \\
\hline 3 & 1 & 2 & 2 & 1 & 1 & 3308.3959 & -0.0086 \\
\hline 4 & 1 & 4 & 3 & 1 & 3 & 4121.7280 & -0.0046 \\
\hline 4 & 0 & 4 & 3 & 0 & 3 & 4230.8245 & -0.0027 \\
\hline 4 & 2 & 3 & 3 & 2 & 2 & 4268.9904 & 0.0035 \\
\hline 4 & 1 & 3 & 3 & 1 & 2 & 4405.8700 & -0.0073 \\
\hline 5 & 0 & 5 & 4 & 0 & 4 & 5259.8393 & -0.0054 \\
\hline 5 & 1 & 4 & 4 & 1 & 3 & 5498.3015 & 0.0018 \\
\hline 6 & 1 & 6 & 5 & 1 & 5 & 6164.8586 & -0.0048 \\
\hline 6 & 0 & 6 & 5 & 0 & 5 & 6274.2962 & 0.0123 \\
\hline 6 & 2 & 4 & 5 & 2 & 3 & 6523.3218 & 0.0072 \\
\hline
\end{tabular}

Table S50. Measured frequencies and residuals (in $\mathrm{MHz}$ ) for the rotational transitions of the ${ }^{13} \mathrm{C}_{2}$ isotopologue of $2 \mathrm{w}-\mathrm{IV}$.

\begin{tabular}{rccccccr}
\hline \hline $\mathrm{J}^{\prime}$ & $\mathrm{K}_{-1}^{\prime}$ & $\mathrm{K}_{+1}^{\prime}$ & $\mathrm{J}^{\prime \prime}$ & $\mathrm{K}_{-1}^{\prime \prime}$ & $\mathrm{K}^{\prime \prime}{ }_{+1}$ & $\mathrm{~V}_{\text {obs }}$ & $\mathrm{V}_{\text {obs }}-\mathrm{V}_{\text {calc }}$ \\
\hline 3 & 0 & 3 & 2 & 0 & 2 & 3202.4890 & 0.0015 \\
3 & 1 & 2 & 2 & 1 & 1 & 3324.6139 & -0.0053 \\
4 & 1 & 4 & 3 & 1 & 3 & 4141.2573 & 0.0019 \\
4 & 0 & 4 & 3 & 0 & 3 & 4250.4781 & 0.0029 \\
4 & 2 & 3 & 3 & 2 & 2 & 4289.6265 & 0.0040 \\
4 & 1 & 3 & 3 & 1 & 2 & 4427.3472 & 0.0007 \\
5 & 1 & 5 & 4 & 1 & 4 & 5169.4734 & 0.0112 \\
5 & 1 & 4 & 4 & 1 & 3 & 5524.8714 & -0.0007 \\
6 & 1 & 6 & 5 & 1 & 5 & 6193.7272 & -0.0118 \\
6 & 0 & 6 & 5 & 0 & 5 & 6302.2768 & 0.0112 \\
6 & 2 & 5 & 5 & 2 & 4 & 6419.9490 & -0.0054 \\
7 & 0 & 7 & 6 & 0 & 6 & 7308.6025 & -0.0088 \\
7 & 1 & 6 & 6 & 1 & 5 & 7696.2654 & 0.0003 \\
\hline \hline
\end{tabular}

Table S51. Measured frequencies and residuals (in $\mathrm{MHz}$ ) for the rotational transitions of the ${ }^{13} \mathrm{C}_{3}$ isotopologue of $2 \mathrm{w}-\mathrm{IV}$.

\begin{tabular}{rccccccr}
\hline \hline $\mathrm{J}^{\prime}$ & $\mathrm{K}_{-1}^{\prime}$ & $\mathrm{K}^{\prime}{ }_{+1}$ & $\mathrm{~J}^{\prime \prime}$ & $\mathrm{K}_{-1}^{\prime \prime}$ & $\mathrm{K}_{+1}^{\prime \prime}$ & $\mathrm{V}_{\text {obs }}$ & $\mathrm{V}_{\text {obs }}-\mathrm{V}_{\text {calc }}$ \\
\hline 3 & 1 & 3 & 2 & 1 & 2 & 3112.0081 & -0.0057 \\
3 & 0 & 3 & 2 & 0 & 2 & 3207.7117 & -0.0062 \\
4 & 1 & 4 & 3 & 1 & 3 & 4144.2619 & -0.0113 \\
4 & 0 & 4 & 3 & 0 & 3 & 4255.9212 & -0.0093 \\
4 & 2 & 2 & 3 & 2 & 1 & 4344.0940 & -0.0167 \\
5 & 2 & 4 & 4 & 2 & 3 & 5366.8515 & 0.0028 \\
5 & 1 & 4 & 4 & 1 & 3 & 5540.6118 & 0.0054 \\
6 & 1 & 6 & 5 & 1 & 5 & 6196.9622 & 0.0145 \\
6 & 0 & 6 & 5 & 0 & 5 & 6305.5429 & 0.0008 \\
6 & 2 & 4 & 5 & 2 & 3 & 6578.6178 & 0.0042 \\
6 & 1 & 5 & 5 & 1 & 4 & 6632.8424 & -0.0001 \\
\hline \hline
\end{tabular}


Table S52. Measured frequencies and residuals (in $\mathrm{MHz}$ ) for the rotational transitions of the ${ }^{13} \mathrm{C}_{4}$ isotopologue of $2 \mathrm{w}-\mathrm{IV}$.

\begin{tabular}{rccccccr}
\hline \hline $\mathrm{J}^{\prime}$ & $\mathrm{K}_{-1}^{\prime}$ & $\mathrm{K}^{\prime}{ }_{+1}$ & $\mathrm{~J}^{\prime \prime}$ & $\mathrm{K}^{\prime \prime}{ }_{-1}$ & $\mathrm{~K}^{\prime \prime}{ }_{+1}$ & $\mathrm{~V}_{\text {obs }}$ & $\mathrm{V}_{\text {obs }} \mathrm{V}_{\text {calc }}$ \\
\hline 3 & 0 & 3 & 2 & 0 & 2 & 3213.7268 & -0.0124 \\
3 & 1 & 2 & 2 & 1 & 1 & 3339.4222 & 0.0100 \\
4 & 1 & 4 & 3 & 1 & 3 & 4153.3150 & -0.0020 \\
4 & 0 & 4 & 3 & 0 & 3 & 4264.4886 & -0.0090 \\
4 & 2 & 3 & 3 & 2 & 2 & 4305.6397 & -0.0070 \\
4 & 2 & 2 & 3 & 2 & 1 & 4350.3587 & -0.0042 \\
5 & 2 & 4 & 4 & 2 & 3 & 5376.3407 & 0.0097 \\
5 & 1 & 4 & 4 & 1 & 3 & 5548.6481 & 0.0114 \\
6 & 0 & 6 & 5 & 0 & 5 & 6320.0701 & -0.0019 \\
6 & 1 & 5 & 5 & 1 & 4 & 6642.9478 & -0.0094 \\
7 & 1 & 7 & 6 & 1 & 6 & 7233.5987 & 0.0045 \\
7 & 0 & 7 & 6 & 0 & 6 & 7327.8991 & 0.0038 \\
\hline \hline
\end{tabular}

Table S53. Measured frequencies and residuals (in $\mathrm{MHz}$ ) for the rotational transitions of the ${ }^{13} \mathrm{C}_{5}$ isotopologue of $2 \mathrm{w}-\mathrm{IV}$.

\begin{tabular}{rcccccrr}
\hline \hline $\mathrm{J}^{\prime}$ & $\mathrm{K}_{-1}^{\prime}$ & $\mathrm{K}_{+1}^{\prime}$ & $\mathrm{J}^{\prime \prime}$ & $\mathrm{K}_{-1}^{\prime \prime}$ & $\mathrm{K}_{+1}^{\prime \prime}$ & $\mathrm{V}_{\text {obs }}$ & $\mathrm{V}_{\text {obs }}-\mathrm{V}_{\text {calc }}$ \\
\hline 3 & 1 & 3 & 2 & 1 & 2 & 3122.0629 & 0.0035 \\
3 & 0 & 3 & 2 & 0 & 2 & 3215.3780 & -0.0153 \\
3 & 1 & 2 & 2 & 1 & 1 & 3337.9365 & 0.0044 \\
4 & 1 & 4 & 3 & 1 & 3 & 4158.0292 & 0.0089 \\
4 & 0 & 4 & 3 & 0 & 3 & 4267.6200 & -0.0051 \\
4 & 2 & 3 & 3 & 2 & 2 & 4306.8884 & 0.0004 \\
4 & 1 & 3 & 3 & 1 & 2 & 4445.0739 & -0.0074 \\
5 & 1 & 5 & 4 & 1 & 4 & 5190.3942 & -0.0030 \\
5 & 0 & 5 & 4 & 0 & 4 & 5305.0998 & 0.0006 \\
5 & 2 & 4 & 4 & 2 & 3 & 5378.1579 & 0.0073 \\
5 & 1 & 4 & 4 & 1 & 3 & 5547.0152 & 0.0014 \\
6 & 1 & 6 & 5 & 1 & 5 & 6218.8247 & -0.0071 \\
6 & 0 & 6 & 5 & 0 & 5 & 6327.7693 & 0.0042 \\
6 & 2 & 5 & 5 & 2 & 4 & 6445.8138 & 0.0036 \\
\hline \hline
\end{tabular}

Table S54. Measured frequencies and residuals (in $\mathrm{MHz}$ ) for the rotational transitions of the ${ }^{13} \mathrm{C}_{6}$ isotopologue of $2 \mathrm{w}-\mathrm{IV}$.

\begin{tabular}{|c|c|c|c|c|c|c|c|}
\hline $\mathrm{J}^{\prime}$ & $\mathrm{K}_{-1}^{\prime}$ & $\mathrm{K}^{\prime}+1$ & $\mathrm{~J}^{\prime \prime}$ & $\mathrm{K}^{\prime \prime}{ }_{1}$ & $\mathrm{~K}^{\prime \prime}+1$ & $\mathrm{~V}_{\text {obs }}$ & $\mathrm{V}_{\mathrm{obs}}-\mathrm{V}_{\mathrm{calc}}$ \\
\hline 3 & 0 & 3 & 2 & 0 & 2 & 3215.3781 & -0.0051 \\
\hline 3 & 1 & 2 & 2 & 1 & 1 & 3340.1722 & -0.0010 \\
\hline 4 & 1 & 4 & 3 & 1 & 3 & 4156.1697 & 0.0103 \\
\hline 4 & 0 & 4 & 3 & 0 & 3 & 4266.9758 & -0.0013 \\
\hline 4 & 1 & 3 & 3 & 1 & 2 & 4447.8648 & -0.0117 \\
\hline 5 & 1 & 5 & 4 & 1 & 4 & 5187.8472 & -0.0012 \\
\hline 5 & 0 & 5 & 4 & 0 & 4 & 5303.3896 & -0.0102 \\
\hline 5 & 2 & 4 & 4 & 2 & 3 & 5378.7816 & 0.0041 \\
\hline 6 & 1 & 6 & 5 & 1 & 5 & 6215.4705 & -0.0157 \\
\hline 6 & 2 & 5 & 5 & 2 & 4 & 6446.3093 & 0.0140 \\
\hline 6 & 2 & 4 & 5 & 2 & 3 & 6587.9332 & 0.0023 \\
\hline 7 & 0 & 7 & 6 & 0 & 6 & 7333.7016 & 0.0088 \\
\hline
\end{tabular}

Table S55. Measured frequencies and residuals (in $\mathrm{MHz}$ ) for the rotational transitions of the ${ }^{18} \mathrm{O}_{7}$ isotopologue of $2 \mathrm{w}$-IV.

\begin{tabular}{rccccccr}
\hline \hline $\mathrm{J}^{\prime}$ & $\mathrm{K}_{-1}^{\prime}$ & $\mathrm{K}^{\prime}{ }_{+1}$ & $\mathrm{~J}^{\prime \prime}$ & $\mathrm{K}^{\prime \prime}{ }_{-1}$ & $\mathrm{~K}^{\prime \prime}{ }_{+1}$ & $\mathrm{~V}_{\text {obs }}$ & $\mathrm{V}_{\text {obs }}-\mathrm{V}_{\text {calc }}$ \\
\hline 3 & 1 & 3 & 2 & 1 & 2 & 3108.5421 & 0.0005 \\
3 & 0 & 3 & 2 & 0 & 2 & 3204.9642 & 0.0099 \\
3 & 1 & 2 & 2 & 1 & 1 & 3333.8397 & -0.0030 \\
4 & 1 & 4 & 3 & 1 & 3 & 4139.4929 & 0.0034
\end{tabular}




\begin{tabular}{rrrrrrrr}
4 & 0 & 4 & 3 & 0 & 3 & 4251.6089 & -0.0008 \\
4 & 2 & 3 & 3 & 2 & 2 & 4295.1757 & -0.0024 \\
4 & 2 & 2 & 3 & 2 & 1 & 4342.5370 & 0.0073 \\
4 & 1 & 3 & 3 & 1 & 2 & 4438.9753 & -0.0025 \\
5 & 1 & 5 & 4 & 1 & 4 & 5166.5008 & -0.0026 \\
5 & 0 & 5 & 4 & 0 & 4 & 5282.1981 & -0.0011 \\
5 & 2 & 4 & 4 & 2 & 3 & 5362.9063 & 0.0077 \\
5 & 2 & 3 & 4 & 2 & 2 & 5454.3391 & -0.0012 \\
5 & 1 & 4 & 4 & 1 & 3 & 5538.2336 & -0.0102 \\
6 & 1 & 6 & 5 & 1 & 5 & 6189.2276 & -0.0027 \\
6 & 0 & 6 & 5 & 0 & 5 & 6297.1083 & -0.0019 \\
6 & 2 & 5 & 5 & 2 & 4 & 6426.6135 & -0.0026 \\
6 & 2 & 4 & 5 & 2 & 3 & 6577.6752 & -0.0030 \\
\hline \hline
\end{tabular}

Table S56. Measured frequencies and residuals (in $\mathrm{MHz}$ ) for the rotational transitions of the ${ }^{13} \mathrm{C}_{8}$ isotopologue of $2 \mathrm{w}-\mathrm{IV}$.

\begin{tabular}{rcccccrr}
\hline \hline $\mathrm{J}^{\prime}$ & $\mathrm{K}_{-1}^{\prime}$ & $\mathrm{K}^{\prime}{ }_{+1}$ & $\mathrm{~J}^{\prime \prime}$ & $\mathrm{K}_{-1}{ }_{-1}$ & $\mathrm{~K}^{\prime \prime}{ }_{+1}$ & $\mathrm{~V}_{\text {obs }}$ & $\mathrm{V}_{\text {obs }}-\mathrm{V}_{\text {calc }}$ \\
\hline 3 & 0 & 3 & 2 & 0 & 2 & 3193.1163 & 0.0123 \\
3 & 1 & 2 & 2 & 1 & 1 & 3314.1823 & -0.0139 \\
4 & 0 & 4 & 3 & 0 & 3 & 4238.3698 & 0.0008 \\
4 & 2 & 2 & 3 & 2 & 1 & 4318.3165 & 0.0011 \\
4 & 1 & 3 & 3 & 1 & 2 & 4413.5728 & -0.0026 \\
5 & 0 & 5 & 4 & 0 & 4 & 5269.1660 & -0.0070 \\
5 & 2 & 4 & 4 & 2 & 3 & 5340.5325 & 0.0020 \\
5 & 1 & 4 & 4 & 1 & 3 & 5507.8829 & 0.0035 \\
6 & 1 & 6 & 5 & 1 & 5 & 6176.2735 & -0.0058 \\
6 & 2 & 4 & 5 & 2 & 3 & 6535.2700 & -0.0010 \\
6 & 1 & 5 & 5 & 1 & 4 & 6595.2426 & 0.0046 \\
7 & 1 & 7 & 6 & 1 & 6 & 7193.8471 & 0.0042 \\
\hline \hline
\end{tabular}

Table S57. Measured frequencies and residuals (in $\mathrm{MHz}$ ) for the rotational transitions of the ${ }^{13} \mathrm{C}_{9}$ isotopologue of $2 \mathrm{w}$-IV.

\begin{tabular}{rcccccrr}
\hline \hline $\mathrm{J}^{\prime}$ & $\mathrm{K}_{-1}^{\prime}$ & $\mathrm{K}_{+1}^{\prime}$ & $\mathrm{J}^{\prime \prime}$ & $\mathrm{K}_{-1}^{\prime \prime}$ & $\mathrm{K}_{+1}^{\prime \prime}$ & $\mathrm{V}_{\mathrm{obs}}$ & $\mathrm{V}_{\mathrm{obs}}-\mathrm{V}_{\text {calc }}$ \\
\hline 3 & 0 & 3 & 2 & 0 & 2 & 3204.3338 & -0.0101 \\
4 & 1 & 4 & 3 & 1 & 3 & 4141.5027 & 0.0021 \\
4 & 0 & 4 & 3 & 0 & 3 & 4252.1556 & -0.0029 \\
4 & 2 & 3 & 3 & 2 & 2 & 4292.9331 & 0.0083 \\
5 & 1 & 5 & 4 & 1 & 4 & 5169.4734 & -0.0177 \\
5 & 1 & 4 & 4 & 1 & 3 & 5531.8347 & 0.0014 \\
6 & 1 & 6 & 5 & 1 & 5 & 6193.4296 & 0.0132 \\
6 & 2 & 5 & 5 & 2 & 4 & 6424.3082 & 0.0101 \\
6 & 2 & 4 & 5 & 2 & 3 & 6566.5464 & -0.0017 \\
6 & 1 & 5 & 5 & 1 & 4 & 6622.9439 & -0.0050 \\
7 & 1 & 7 & 6 & 1 & 6 & 7213.2092 & -0.0038 \\
\hline \hline
\end{tabular}


Table S58. Measured frequencies and residuals (in $\mathrm{MHz}$ ) for the rotational transitions of the ${ }^{18} \mathrm{O}_{10}$ isotopologue of $2 \mathrm{w}$-IV.

\begin{tabular}{|c|c|c|c|c|c|c|c|}
\hline $\mathrm{J}^{\prime}$ & $\mathrm{K}_{-1}^{\prime}$ & $\mathrm{K}_{+1}^{\prime}$ & $\mathrm{J}^{\prime \prime}$ & $\mathrm{K}_{-1}^{\prime \prime}$ & $\mathrm{K}_{+1}^{\prime \prime}$ & $V_{\text {obs }}$ & $\mathrm{V}_{\text {obs }}-\mathrm{V}_{\text {calc }}$ \\
\hline 3 & 1 & 3 & 2 & 1 & 2 & 3033.0624 & -0.0008 \\
\hline 3 & 0 & 3 & 2 & 0 & 2 & 3122.8909 & -0.0005 \\
\hline 3 & 2 & 2 & 2 & 2 & 1 & 3138.5853 & -0.0002 \\
\hline 3 & 2 & 1 & 2 & 2 & 0 & 3154.2839 & -0.0021 \\
\hline 3 & 1 & 2 & 2 & 1 & 1 & 3239.1779 & 0.0004 \\
\hline 4 & 1 & 4 & 3 & 1 & 3 & 4039.7866 & -0.0019 \\
\hline 4 & 0 & 4 & 3 & 0 & 3 & 4146.0711 & -0.0009 \\
\hline 4 & 2 & 3 & 3 & 2 & 2 & 4181.7013 & 0.0023 \\
\hline 4 & 3 & 1 & 3 & 3 & 0 & 4193.0914 & 0.0009 \\
\hline 4 & 2 & 2 & 3 & 2 & 1 & 4220.3998 & -0.0005 \\
\hline 4 & 1 & 3 & 3 & 1 & 2 & 4313.9460 & -0.0014 \\
\hline 5 & 1 & 5 & 4 & 1 & 4 & 5043.2333 & -0.0002 \\
\hline 5 & 0 & 5 & 4 & 0 & 4 & 5155.6726 & 0.0018 \\
\hline 5 & 2 & 4 & 4 & 2 & 3 & 5222.1797 & -0.0009 \\
\hline 5 & 3 & 2 & 4 & 3 & 1 & 5246.0256 & 0.0011 \\
\hline 5 & 2 & 3 & 4 & 2 & 2 & 5297.4275 & -0.0017 \\
\hline 5 & 1 & 4 & 4 & 1 & 3 & 5384.0330 & -0.0016 \\
\hline 6 & 1 & 6 & 5 & 1 & 5 & 6043.0432 & -0.0008 \\
\hline 6 & 1 & 6 & 5 & 1 & 5 & 6043.0432 & -0.0008 \\
\hline 6 & 0 & 6 & 5 & 0 & 5 & 6151.3551 & -0.0004 \\
\hline 6 & 3 & 4 & 5 & 3 & 3 & 6295.0299 & -0.0018 \\
\hline 6 & 3 & 3 & 5 & 3 & 2 & 6302.8575 & 0.0001 \\
\hline 6 & 2 & 4 & 5 & 2 & 3 & 6385.0073 & 0.0014 \\
\hline 7 & 1 & 7 & 6 & 1 & 6 & 7039.1119 & -0.0012 \\
\hline 7 & 0 & 7 & 6 & 0 & 6 & 7135.1665 & 0.0041 \\
\hline 7 & 2 & 6 & 6 & 2 & 5 & 7292.7479 & 0.0001 \\
\hline 7 & 3 & 5 & 6 & 3 & 4 & 7347.7496 & 0.0014 \\
\hline 7 & 2 & 5 & 6 & 2 & 4 & 7480.3497 & 0.0029 \\
\hline 7 & 1 & 6 & 6 & 1 & 5 & 7503.0049 & -0.0021 \\
\hline
\end{tabular}


Table S59. Measured frequencies and residuals (in $\mathrm{MHz}$ ) for the rotational transitions of the ${ }^{18} \mathrm{O}_{11}$ isotopologue of $2 \mathrm{w}$-IV.

\begin{tabular}{|c|c|c|c|c|c|c|c|}
\hline $\mathrm{J}^{\prime}$ & $\mathrm{K}^{\prime}{ }_{-1}$ & $\mathrm{~K}^{\prime}+1$ & J" & $\mathrm{K}^{\prime \prime}{ }_{-1}$ & $\mathrm{~K}^{\prime \prime}+1$ & $\mathrm{~V}_{\text {obs }}$ & $\mathrm{V}_{\text {obs }}-\mathrm{V}_{\text {calc }}$ \\
\hline 3 & 1 & 3 & 2 & 1 & 2 & 3057.5172 & 0.0002 \\
\hline 3 & 0 & 3 & 2 & 0 & 2 & 3147.5081 & 0.0000 \\
\hline 3 & 2 & 2 & 2 & 2 & 1 & 3163.4253 & -0.0040 \\
\hline 3 & 2 & 1 & 2 & 2 & 0 & 3179.3507 & -0.0061 \\
\hline 3 & 1 & 2 & 2 & 1 & 1 & 3264.3391 & 0.0000 \\
\hline 4 & 1 & 4 & 3 & 1 & 3 & 4072.3343 & -0.0002 \\
\hline 4 & 0 & 4 & 3 & 0 & 3 & 4178.6484 & -0.0009 \\
\hline 4 & 2 & 3 & 3 & 2 & 2 & 4214.7790 & -0.0002 \\
\hline 4 & 3 & 2 & 3 & 3 & 1 & 4225.4761 & 0.0052 \\
\hline 4 & 3 & 1 & 3 & 3 & 0 & 4226.3415 & -0.0006 \\
\hline 4 & 2 & 2 & 3 & 2 & 1 & 4254.0277 & -0.0003 \\
\hline 4 & 1 & 3 & 3 & 1 & 2 & 4347.4189 & -0.0012 \\
\hline 5 & 1 & 5 & 4 & 1 & 4 & 5083.8318 & 0.0005 \\
\hline 5 & 0 & 5 & 4 & 0 & 4 & 5196.0549 & -0.0009 \\
\hline 5 & 2 & 4 & 4 & 2 & 3 & 5263.4500 & -0.0092 \\
\hline 5 & 3 & 3 & 4 & 3 & 2 & 5284.6318 & -0.0014 \\
\hline 5 & 3 & 2 & 4 & 3 & 1 & 5287.6690 & 0.0006 \\
\hline 5 & 2 & 3 & 4 & 2 & 2 & 5339.7277 & -0.0001 \\
\hline 5 & 1 & 4 & 4 & 1 & 3 & 5425.7394 & -0.0026 \\
\hline 6 & 1 & 6 & 5 & 1 & 5 & 6091.6539 & -0.0002 \\
\hline 6 & 0 & 6 & 5 & 0 & 5 & 6199.4499 & 0.0001 \\
\hline 6 & 2 & 5 & 5 & 2 & 4 & 6308.8264 & -0.0005 \\
\hline 6 & 3 & 4 & 5 & 3 & 3 & 6344.9445 & 0.0035 \\
\hline 6 & 3 & 3 & 5 & 3 & 2 & 6352.9654 & -0.0012 \\
\hline 6 & 2 & 4 & 5 & 2 & 3 & 6436.0237 & -0.0001 \\
\hline 7 & 1 & 7 & 6 & 1 & 6 & 7095.7008 & -0.0011 \\
\hline 7 & 0 & 7 & 6 & 0 & 6 & 7190.9654 & 0.0014 \\
\hline 7 & 2 & 6 & 6 & 2 & 5 & 7350.2762 & 0.0005 \\
\hline 7 & 3 & 5 & 6 & 3 & 4 & 7405.9902 & 0.0009 \\
\hline 7 & 2 & 5 & 6 & 2 & 4 & 7540.0249 & 0.0016 \\
\hline 7 & 1 & 6 & 6 & 1 & 5 & 7560.8194 & 0.0014 \\
\hline
\end{tabular}

